\title{
NONLINEAR SPECTRAL CALCULUS AND SUPER-EXPANDERS
}

\author{
MANOR MENDEL AND ASSAF NAOR
}

\begin{abstract}
Nonlinear spectral gaps with respect to uniformly convex normed spaces are shown to satisfy a spectral calculus inequality that establishes their decay along Cesàro averages. Nonlinear spectral gaps of graphs are also shown to behave sub-multiplicatively under zigzag products. These results yield a combinatorial construction of super-expanders, i.e., a sequence of 3-regular graphs that does not admit a coarse embedding into any uniformly convex normed space.
\end{abstract}

\section{Contents}

1. Introduction

1.1. Coarse non-embeddability

1.2. Absolute spectral gaps

1.3. A combinatorial approach to the existence of super-expanders

1.3.1. The iterative strategy

1.3.2. The need for a calculus for nonlinear spectral gaps

1.3.3. Metric Markov cotype and spectral calculus

1.3.4. The base graph

1.3.5. Sub-multiplicativity theorems for graph products

2. Preliminary results on nonlinear spectral gaps

2.1. The trivial bound for general graphs

2.2. $\gamma$ versus $\gamma_{+}$

2.3. Edge completion

3. Metric Markov cotype implies nonlinear spectral calculus

4. An iterative construction of super-expanders

5. The heat semigroup on the tail space

5.1. Warmup: the tail space of scalar valued functions

5.2. Proof of Theorem 5.1

5.3. Proof of Theorem 5.2

5.4. Inverting the Laplacian on the vector-valued tail space

6. Nonlinear spectral gaps in uniformly convex normed spaces

6.1. Norm bounds need not imply nonlinear spectral gaps

6.2. A partial converse to Lemma 6.1 in uniformly convex spaces

6.3. Martingale inequalities and metric Markov cotype

7. Construction of the base graph

8. Graph products

8.1. Sub-multiplicativity for tensor products

8.2. Sub-multiplicativity for the zigzag product

8.3. Sub-multiplicativity for replacement products

8.4. Sub-multiplicativity for derandomized squaring

9. Counterexamples

9.1. Expander families need not embed coarsely into each other

9.2. A metric space failing calculus for nonlinear spectral gaps

References 


\section{INTRODUCTION}

Let $A=\left(a_{i j}\right)$ be an $n \times n$ symmetric stochastic matrix and let

$$
1=\lambda_{1}(A) \geqslant \lambda_{2}(A) \geqslant \cdots \geqslant \lambda_{n}(A) \geqslant-1
$$

be its eigenvalues. The reciprocal of the spectral gap of $A$, i.e., the quantity $\frac{1}{1-\lambda_{2}(A)}$, is the smallest $\gamma \in(0, \infty]$ such that for every $x_{1}, \ldots, x_{n} \in \mathbb{R}$ we have

$$
\frac{1}{n^{2}} \sum_{i=1}^{n} \sum_{j=1}^{n}\left(x_{i}-x_{j}\right)^{2} \leqslant \frac{\gamma}{n} \sum_{i=1}^{n} \sum_{j=1}^{n} a_{i j}\left(x_{i}-x_{j}\right)^{2} .
$$

By summing over the coordinates with respect to some orthonormal basis, a restatement of (1) is that $\frac{1}{1-\lambda_{2}(A)}$ is the smallest $\gamma \in(0, \infty]$ such that for all $x_{1}, \ldots, x_{n} \in L_{2}$ we have

$$
\frac{1}{n^{2}} \sum_{i=1}^{n} \sum_{j=1}^{n}\left\|x_{i}-x_{j}\right\|_{2}^{2} \leqslant \frac{\gamma}{n} \sum_{i=1}^{n} \sum_{j=1}^{n} a_{i j}\left\|x_{i}-x_{j}\right\|_{2}^{2} .
$$

It is natural to generalize (2) in several ways: one can replace the exponent 2 by some other exponent $p>0$ and, much more substantially, one can replace the Euclidean geometry by some other metric space $\left(X, d_{X}\right)$. Such generalizations are standard practice in metric geometry. For the sake of presentation, it is beneficial to take this generalization to even greater extremes, as follows. Let $X$ be an arbitrary set and let $K: X \times X \rightarrow[0, \infty)$ be a symmetric function. Such functions are sometimes called kernels in the literature, and we shall adopt this terminology here. Define the reciprocal spectral gap of $A$ with respect to $K$, denoted $\gamma(A, K)$, to be the infimum over those $\gamma \in(0, \infty]$ such that for all $x_{1}, \ldots, x_{n} \in X$ we have

$$
\frac{1}{n^{2}} \sum_{i=1}^{n} \sum_{j=1}^{n} K\left(x_{i}, x_{j}\right) \leqslant \frac{\gamma}{n} \sum_{i=1}^{n} \sum_{j=1}^{n} a_{i j} K\left(x_{i}, x_{j}\right) .
$$

In what follows we will also call $\gamma(A, K)$ the Poincaré constant of the matrix $A$ with respect to the kernel $K$. Readers are encouraged to focus on the geometrically meaningful case when $K$ is a power of some metric on $X$, though as will become clear presently, a surprising amount of ground can be covered without any assumption on the kernel $K$.

For concreteness we restate the above discussion: the standard gap in the linear spectrum of $A$ corresponds to considering Poincaré constants with respect to Euclidean spaces (i.e., kernels which are squares of Euclidean metrics), but there is scope for a theory of nonlinear spectral gaps when one considers inequalities such as (3) with respect to other geometries. The purpose of this paper is to make progress towards such a theory, with emphasis on possible extensions of spectral calculus to nonlinear (non-Euclidean) settings. We apply our results on calculus for nonlinear spectral gaps to construct new strong types of expander graphs, and to resolve a question of V. Lafforgue [28]. We obtain a combinatorial construction of a remarkable type of bounded degree graphs whose shortest path metric is incompatible with the geometry of any uniformly convex normed space in a very strong sense (i.e., coarse non-embeddability). The existence of such graph families was first discovered by Lafforgue via a tour de force algebraic construction [28]. Our work indicates that there is hope for a useful and rich theory of nonlinear spectral gaps, beyond the sporadic (though often highly nontrivial) examples that have been previously studied in the literature. 
1.1. Coarse non-embeddability. A sequence of metric spaces $\left\{\left(X_{n}, d_{X_{n}}\right)\right\}_{n=1}^{\infty}$ is said to embed coarsely (with the same moduli) into a metric space $\left(Y, d_{Y}\right)$ if there exist two nondecreasing functions $\alpha, \beta:[0, \infty) \rightarrow[0, \infty)$ such that $\lim _{t \rightarrow \infty} \alpha(t)=\infty$, and there exist mappings $f_{n}: X_{n} \rightarrow Y$, such that for all $n \in \mathbb{N}$ and $x, y \in X_{n}$ we have

$$
\alpha\left(d_{X_{n}}(x, y)\right) \leqslant d_{Y}\left(f_{n}(x), f_{n}(y)\right) \leqslant \beta\left(d_{X_{n}}(x, y)\right) .
$$

(44) is a weak form of "metric faithfulness" of the mappings $f_{n}$; a seemingly humble requirement that can be restated informally as "large distances map uniformly to large distances". Nevertheless, this weak notion of embedding (much weaker than, say, bi-Lipschitz embeddability) has beautiful applications in geometry and group theory; see [18, 68, 12, 65, 20] and the references therein for examples of such applications.

Since coarse embeddability is a weak requirement, it is quite difficult to prove coarse nonembeddability. Very few methods to establish such a result are known, among which is the use of nonlinear spectral gaps, as pioneered by Gromov [19] (other such methods include coarse notions of metric dimension [18, or the use of metric cotype [43]. These methods do not seem to be applicable to the question that we study here). Gromov's argument is simple: fix $d \in \mathbb{N}$ and suppose that $X_{n}=\left(V_{n}, E_{n}\right)$ are connected $d$-regular graphs and that $d_{X_{n}}(\cdot, \cdot)$ is the shortest-path metric induced by $X_{n}$ on $V_{n}$. Suppose also that there exist $p, \gamma \in(0, \infty)$ such that for every $n \in \mathbb{N}$ and $f: V_{n} \rightarrow Y$ we have

$$
\frac{1}{\left|V_{n}\right|^{2}} \sum_{(u, v) \in V_{n} \times V_{n}} d_{Y}(f(u), f(v))^{p} \leqslant \frac{\gamma}{d\left|V_{n}\right|} \sum_{(x, y) \in E_{n}} d_{Y}(f(x), f(y))^{p} .
$$

A combination of (44) and (5) yields the bound

$$
\frac{1}{\left|V_{n}\right|^{2}} \sum_{(u, v) \in V_{n} \times V_{n}} \alpha\left(d_{X_{n}}(u, v)\right)^{p} \leqslant \gamma \beta(1)^{p} .
$$

But, since $X_{n}$ is a bounded degree graph, at least half of the pairs of vertices $(u, v) \in V_{n} \times V_{n}$ satisfy $d_{X_{n}}(u, v) \geqslant c_{d} \log \left|V_{n}\right|$, where $c_{d} \in(0, \infty)$ depends on the degree $d$ but not on $n$. Thus $\alpha\left(c_{d} \log \left|V_{n}\right|\right)^{p} \leqslant 2 \gamma \beta(1)^{p}$, and in particular if $\lim _{n \rightarrow \infty}\left|V_{n}\right|=\infty$ then we get a contradiction to the assumption $\lim _{t \rightarrow \infty} \alpha(t)=\infty$. Observe in passing that this argument also shows that the metric space $\left(X_{n}, d_{X_{n}}\right)$ has bi-Lipschitz distortion $\Omega\left(\log \left|V_{n}\right|\right)$ in $Y$; such an argument was first used by Linial, London and Rabinovich [34] (see also [39]) to show that Bourgain's embedding theorem [10] is asymptotically sharp.

Assumption (5) can be restated as saying that $\gamma\left(A_{n}, d_{Y}^{p}\right) \leqslant \gamma$, where $A_{n}$ is the normalized adjacency matrix of $X_{n}$. This condition can be viewed to mean that the graphs $\left\{X_{n}\right\}_{n=1}^{\infty}$ are "expanders" with respect to $\left(Y, d_{Y}\right)$. Note that if $Y$ contains at least two points then (5) implies that $\left\{X_{n}\right\}_{n=1}^{\infty}$ are necessarily also expanders in the classical sense (see [21, 35] for more on classical expanders).

A key goal in the coarse non-embeddability question is therefore to construct such $\left\{X_{n}\right\}_{n=1}^{\infty}$ for which one can prove the inequality (5) for non-Hilbertian targets $Y$. This question has been previously investigated by several authors. Matoušek [39] devised an extrapolation method for Poincaré inequalities (see also the description of Matoušek's argument in [6]) which establishes the validity of (5) for every expander when $Y=L_{p}$. Works of Ozawa [55] and Pisier [57, 60] prove (5) for every expander if $Y$ is Banach space which satisfies certain geometric conditions (e.g. $Y$ can be taken to be a Banach lattice of finite cotype; see [33] for background on these notions). In [54, 51] additional results of this type are obtained. 
A normed space is called super-reflexive if it admits an equivalent norm which is uniformly convex. Recall that a normed space $\left(X,\|\cdot\|_{X}\right)$ is uniformly convex if for every $\varepsilon \in(0,1)$ there exists $\delta=\delta_{X}(\varepsilon)>0$ such that for any two vectors $x, y \in X$ with $\|x\|_{X}=\|y\|_{X}=1$ and $\|x-y\|_{X} \geqslant \varepsilon$ we have $\left\|\frac{x+y}{2}\right\|_{X} \leqslant 1-\delta$. The question whether there exists a sequence of arbitrarily large regular graphs of bounded degree which do not admit a coarse embedding into any super-reflexive normed space was posed by Kasparov and $\mathrm{Yu}$ in [26], and was solved in the remarkable work of V. Lafforgue [28] on the strengthened version of property $(T)$ for $S L_{3}(\mathbb{F})$ when $\mathbb{F}$ is a non-Archimedian local field (see also [3, 30]). Thus, for concreteness, Lafforgue's graphs can be obtained as Cayley graphs of finite quotients of co-compact lattices in $S L_{3}\left(\mathbb{Q}_{p}\right)$, where $p$ is a prime and $\mathbb{Q}_{p}$ is the $p$-adic rationals. The potential validity of the same property for finite quotients of $S L_{3}(\mathbb{Z})$ remains an intriguing open question [28].

Here we obtain a different solution of the Kasparov-Yu problem via a new approach that uses the zigzag product of Reingold, Vadhan, and Wigderson [64], as well as a variety of analytic and geometric arguments of independent interest. More specifically, we construct a family of 3-regular graphs that satisfies (5) for every super-reflexive Banach space $X$ (where $\gamma$ depends only on the geometry $X$ ); such graphs are called super-expanders.

Theorem 1.1 (Existence of super-expanders). There exists a sequence of 3-regular graphs $\left\{G_{n}=\left(V_{n}, E_{n}\right)\right\}_{n=1}^{\infty}$ such that $\lim _{n \rightarrow \infty}\left|V_{n}\right|=\infty$ and for every super-reflexive Banach space $\left(X,\|\cdot\|_{X}\right)$ we have

$$
\sup _{n \in \mathbb{N}} \gamma\left(A_{G_{n}},\|\cdot\|_{X}^{2}\right)<\infty,
$$

where $A_{G_{n}}$ is the normalized adjacency matrix of $G_{n}$.

As we explained earlier, the existence of super-expanders was previously proved by Lafforgue [28]. Theorem 1.1 yields a second construction of such graphs (no other examples are currently known). Our proof of Theorem 1.1 is entirely different from Lafforgue's approach: it is based on a new systematic investigation of nonlinear spectral gaps and an elementary procedure which starts with a given small graph and iteratively increases its size so as to obtain the desired graph sequence. In fact, our study of nonlinear spectral gaps constitutes the main contribution of this work, and the new solution of the Kasparov-Yu problem should be viewed as an illustration of the applicability of our analytic and geometric results, which will be described in detail presently.

We state at the outset that it is a major open question whether every expander graph sequence satisfies (5) for every uniformly convex normed space $X$. It is also unknown whether there exist graph families of bounded degree and logarithmic girth that do not admit a coarse embedding into any super-reflexive normed space; this question is of particular interest in the context of the potential application to the Novikov conjecture that was proposed by Kasparov and $\mathrm{Yu}$ in [26], since it would allow one to apply Gromov's random group construction [19] with respect to actions on super-reflexive spaces.

Some geometric restriction on the target space $X$ must be imposed in order for it to admit a sequence of expanders. Indeed, the relation between nonlinear spectral gaps and coarse non-embeddability, in conjunction with the fact that every finite metric space embeds isometrically into $\ell_{\infty}$, shows that (for example) $X=\ell_{\infty}$ can never satisfy (5) for a family of graphs of bounded degree and unbounded cardinality. We conjecture that for a normed space $X$ the existence of such a graph family is equivalent to having finite cotype, i.e., that there 
exists $\varepsilon_{0} \in(0, \infty)$ and $n_{0} \in \mathbb{N}$ such that any embedding of $\ell_{\infty}^{n_{0}}$ into $X$ incurs bi-Lipschitz distortion at least $1+\varepsilon_{0}$; see e.g. [40] for background on this notion.

Our approach can also be used (see Remark 4.4 below) to show that there exist bounded degree graph sequences which do not admit a coarse embedding into any $K$-convex normed space. A normed space $X$ is $K$-convex 1 . if there exists $\varepsilon_{0}>0$ and $n_{0} \in \mathbb{N}$ such that any embedding of $\ell_{1}^{n_{0}}$ into $X$ incurs distortion at least $1+\varepsilon_{0}$; see [58]. The question whether such graph sequences exist was asked by Lafforgue [28]. Independently of our work, Lafforgue [29] succeeded to modify his argument so as to prove the desired coarse non-embeddability into $K$-convex spaces for his graph sequence as well.

1.2. Absolute spectral gaps. The parameter $\gamma(A, K)$ will reappear later, but for several purposes we need to first study a variant of it which corresponds to the absolute spectral gap of a matrix. Define

$$
\lambda(A) \stackrel{\text { def }}{=} \max _{i \in\{2, \ldots, n\}}\left|\lambda_{i}(A)\right|,
$$

and call the quantity $1-\lambda(A)$ the absolute spectral gap of $A$. Similarly to (2), the reciprocal of the absolute spectral gap of $A$ is the smallest $\gamma_{+} \in(0, \infty]$ such that for all $x_{1}, \ldots, x_{n}, y_{1}, \ldots, y_{n} \in L_{2}$ we have

$$
\frac{1}{n^{2}} \sum_{i=1}^{n} \sum_{j=1}^{n}\left\|x_{i}-y_{j}\right\|_{2}^{2} \leqslant \frac{\gamma_{+}}{n} \sum_{i=1}^{n} \sum_{j=1}^{n} a_{i j}\left\|x_{i}-y_{j}\right\|_{2}^{2} .
$$

Analogously to (3), given a kernel $K: X \times X \rightarrow[0, \infty)$ we can then define $\gamma_{+}(A, K)$ to be the the infimum over those $\gamma_{+} \in(0, \infty]$ such that for all $x_{1}, \ldots, x_{n}, y_{1}, \ldots, y_{n} \in X$ we have

$$
\frac{1}{n^{2}} \sum_{i=1}^{n} \sum_{j=1}^{n} K\left(x_{i}, y_{j}\right) \leqslant \frac{\gamma_{+}}{n} \sum_{i=1}^{n} \sum_{j=1}^{n} a_{i j} K\left(x_{i}, y_{j}\right) .
$$

Note that clearly $\gamma_{+}(A, K) \geqslant \gamma(A, K)$. Additional useful relations between $\gamma(\cdot, \cdot)$ and $\gamma_{+}(\cdot, \cdot)$ are discussed in Section 2.2 .

1.3. A combinatorial approach to the existence of super-expanders. In what follows we will often deal with finite non-oriented regular graphs, which will always be allowed to have self loops and multiple edges (note that the shortest-path metric is not influenced by multiple edges or self loops). When discussing a graph $G=(V, E)$ it will always be understood that $V$ is a finite set and $E$ is a multi-subset of the ordered pairs $V \times V$, i.e., each ordered pair $(u, v) \in V \times V$ is allowed to appear in $E$ multiple times? 2 . We also always impose the condition $(u, v) \in E \Longrightarrow(v, u) \in E$, corresponding to the fact that $G$ is not oriented. For $(u, v) \in V \times V$ we denote by $E(u, v)=E(v, u)$ the number of times that $(u, v)$ appears in $E$. Thus, the graph $G$ is completely determined by the integer matrix $(E(u, v))_{(u, v) \in V \times V}$. The degree of $u \in V$ is $\operatorname{deg}_{G}(u)=\sum_{v \in V} E(u, v)$. Under this convention each self loop contributes 1 to the degree of a vertex. For $d \in \mathbb{N}$, a graph $G=(V, E)$ is $d$-regular if $\operatorname{deg}_{G}(u)=d$ for every $u \in V$. The normalized adjacency matrix of a $d$-regular

\footnotetext{
${ }^{1} K$-convexity is also equivalent to $X$ having Rademacher type strictly bigger than 1 , see [47, 40]. The $K$-convexity property is strictly weaker than super-reflexivity, see [22, 24, 23, 61.

${ }^{2}$ Formally, one can alternatively think of $E$ as a subset of $(V \times V) \times \mathbb{N}$, with the understanding that for $(u, v) \in V \times V$, if we write $J=\{j \in \mathbb{N}:((u, v), j) \in E\}$ then $\{(u, v)\} \times J$ are the $|J|$ "copies" of $(u, v)$ that appear in $E$. However, it will not be necessary to use such formal notation in what follows.
} 
graph $G=(V, E)$, denoted $A_{G}$, is defined as usual by letting its entry at $(u, v) \in V \times V$ be equal to $E(u, v) / d$. When discussing Poincaré constants we will interchangeably identify $G$ with $A_{G}$. Thus, for examples, we write $\lambda(G)=\lambda\left(A_{G}\right)$ and $\gamma_{+}(G, K)=\gamma_{+}\left(A_{G}, K\right)$.

The starting point of our work is an investigation of the behavior of the quantity $\gamma_{+}(G, K)$ under certain graph products, the most important of which (for our purposes) is the zigzag product of Reingold, Vadhan and Wigderson [64. We argue below that such combinatorial constructions are well-adapted to controlling the nonlinear quantity $\gamma_{+}(G, K)$. This crucial fact allows us to use them in a perhaps unexpected geometric context.

1.3.1. The iterative strategy. Reingold, Vadhan and Wigderson 64 introduced the zigzag product of graphs, and used it to produce a novel deterministic construction of expanders. Fix $n_{1}, d_{1}, d_{2} \in \mathbb{N}$. Let $G_{1}$ be a graph with $n_{1}$ vertices which is $d_{1}$-regular and let $G_{2}$ be a graph with $d_{1}$ vertices which is $d_{2}$-regular. The zigzag product $G_{1}$ (2) $G_{2}$ is a graph with $n_{1} d_{1}$ vertices and degree $d_{2}^{2}$, for which the following fundamental theorem is proved in 64].

Theorem 1.2 (Reingold, Vadhan and Wigderson). There exists $f:[0,1] \times[0,1] \rightarrow[0,1]$ satisfying

$$
\forall t \in(0,1), \quad \limsup _{s \rightarrow 0} f(s, t)<1,
$$

such that for every $n_{1}, d_{1}, d_{2} \in \mathbb{N}$, if $G_{1}$ is a graph with $n_{1}$ vertices which is $d_{1}$-regular and $G_{2}$ is a graph with $d_{2}$ vertices which is $d_{2}$-regular then

$$
\lambda\left(G_{1}\left(\mathrm{Z} G_{2}\right) \leqslant f\left(\lambda\left(G_{1}\right), \lambda\left(G_{2}\right)\right) .\right.
$$

The definition of $G_{1}$ (2) $G_{2}$ is recalled in Section 8 . For the purpose of expander constructions one does not need to know anything about the zigzag product other than that it has $n_{1} d_{1}$ vertices and degree $d_{2}^{2}$, and that it satisfies Theorem 1.2, Also, 64 contains explicit algebraic expressions for functions $f$ for which Theorem 1.2 holds true, but we do not need to quote them here because they are irrelevant to the ensuing discussion.

In order to proceed it would be instructive to briefly recall how Reingold, Vadhan and Wigderson used [64] Theorem 1.2 to construct expanders; see also the exposition in Section 9.2 of [21].

Let $H$ be a regular graph with $n_{0}$ vertices and degree $d_{0}$, such that $\lambda(H)<1$. Such a graph $H$ will be called a base graph in what follows. From (8) we deduce that there exist $\varepsilon, \delta \in(0,1)$ such that

$$
s \in(0, \delta) \Longrightarrow f(s, \lambda(H))<1-\varepsilon \text {. }
$$

Fix $t_{0} \in \mathbb{N}$ satisfying

$$
\max \left\{\lambda(H)^{2 t_{0}},(1-\varepsilon)^{t_{0}}\right\}<\delta .
$$

For a graph $G=(V, E)$ and for $t \in \mathbb{N}$, let $G^{t}$ be the graph in which an edge between $u, v \in V$ is drawn for every walk in $G$ of length $t$ whose endpoints are $u, v$. Thus $A_{G^{t}}=\left(A_{G}\right)^{t}$, and if $G$ is $d$-regular then $G^{t}$ is $d^{t}$-regular.

Assume from now on that $n_{0}=d_{0}^{2 t_{0}}$. Define $G_{1}=H^{2}$ and inductively

$$
G_{i+1}=G_{i}^{t_{0}}(2) H \text {. }
$$

Then for all $i \in \mathbb{N}$ the graph $G_{i}$ is well defined and has $n_{0}^{i}=d_{0}^{2 i t_{0}}$ vertices and degree $d_{0}^{2}$. We claim that $\lambda\left(G_{i}\right) \leqslant \max \left\{\lambda(H)^{2}, 1-\varepsilon\right\}$ for all $i \in \mathbb{N}$. Indeed, there is nothing to prove for $i=1$, and if the desired bound is true for $i$ then (11) implies that $\lambda\left(G_{i}^{t_{0}}\right)=\lambda\left(G_{i}\right)^{t_{0}}<\delta$, which by (9) and (10) implies that $\lambda\left(G_{i+1}\right) \leqslant f\left(\lambda\left(G_{i}^{t_{0}}\right), \lambda(H)\right)<1-\varepsilon$. 
Our strategy is to attempt to construct super-expanders via a similar iterative approach. It turns out that obtaining a non-Euclidean version of Theorem 1.2 (which is the seemingly most substantial ingredient of the construction of Reingold, Vadhan and Wigderson) is not an obstacle here due to the following general result.

Theorem 1.3 (Zigzag sub-multiplicativity). Let $G_{1}=\left(V_{1}, E_{1}\right)$ be an $n_{1}$-vertex graph which is $d_{1}$-regular and let $G_{2}=\left(V_{2}, E_{2}\right)$ be a $d_{1}$-vertex graph which is $d_{2}$-regular. Then every kernel $K: X \times X \rightarrow[0, \infty)$ satisfies

$$
\gamma_{+}\left(G_{1}(\mathrm{Z}) G_{2}, K\right) \leqslant \gamma_{+}\left(G_{1}, K\right) \cdot \gamma_{+}\left(G_{2}, K\right)^{2} .
$$

In the special case $X=\mathbb{R}$ and $K(x, y)=(x-y)^{2}$, Theorem 1.3 becomes

$$
\frac{1}{1-\lambda\left(G_{1}(\mathrm{Z}) G_{2}\right)} \leqslant \frac{1}{1-\lambda\left(G_{1}\right)} \cdot \frac{1}{\left(1-\lambda\left(G_{2}\right)\right)^{2}},
$$

implying Theorem 1.2. Note that the explicit bound on the function $f$ of Theorem 1.2 that follows from (13) coincides with the later bound of Reingold, Trevisan and Vadhan [63]. In [64] an improved bound for $\lambda\left(G_{1}\right.$ (2) $\left.G_{2}\right)$ is obtained which is better than the bound of [63] (and hence also (13)), though this improvement in lower-order terms has not been used (so far) in the literature. Theorem 1.3 shows that the fact that the zigzag product preserves spectral gaps has nothing to do with the underlying Euclidean geometry (or linear algebra) that was used in [64, 63]: this is a truly nonlinear phenomenon which holds in much greater generality, and simply amounts to an iteration of the Poincaré inequality (7).

Due to Theorem 1.3 there is hope to carry out an iterative construction based on the zigzag product in great generality. However, this cannot work for all kernels since general kernels can fail to admit a sequence of bounded degree expanders. There are two major obstacles that need to be overcome. The first obstacle is the existence of a base graph, which is a substantial issue whose discussion is deferred to Section 1.3.4. The following subsection describes the main obstacle to our nonlinear zigzag strategy.

1.3.2. The need for a calculus for nonlinear spectral gaps. In the above description of the Reingold-Vadhan-Wigderson iteration we tacitly used the identity $\lambda\left(A^{t}\right)=\lambda(A)^{t}(t \in \mathbb{N})$ in order to increase the spectral gap of $G_{i}$ in each step of the iteration. While this identity is a trivial corollary of spectral calculus, and was thus the "trivial part" of the construction in [64], there is no reason to expect that $\gamma_{+}\left(A^{t}, K\right)$ decreases similarly with $t$ for nonEuclidean kernels $K: X \times X \rightarrow[0, \infty)$. To better grasp what is happening here let us examine the asymptotic behavior of $\gamma_{+}\left(A^{t},|\cdot|^{2}\right)$ as a function of $t$ (here and in what follows $|\cdot|$ denotes the absolute value on $\mathbb{R})$.

$$
\begin{aligned}
\gamma_{+}\left(A^{t},|\cdot|^{2}\right)=\frac{1}{1-\lambda\left(A^{t}\right)}=\frac{1}{1-\lambda(A)^{t}} & \\
& =\frac{1}{1-\left(1-\frac{1}{\gamma_{+}\left(A,|\cdot|^{2}\right)}\right)^{t}} \asymp \max \left\{1, \frac{\gamma_{+}\left(A,|\cdot|^{2}\right)}{t}\right\},
\end{aligned}
$$

where above, and in what follows, $\asymp$ denotes equivalence up to universal multiplicative constants (we will also use the notation $\lesssim, \gtrsim$ to express the corresponding inequalities up to universal constants). (14) means that raising a matrix to a large power $t \in \mathbb{N}$ corresponds to decreasing its (real) Poincaré constant by a factor of $t$ as long as it is possible to do so. 
For our strategy to work for other kernels $K: X \times X \rightarrow[0, \infty)$ we would like $K$ to satisfy a "spectral calculus" inequality of this type, i.e., an inequality which ensures that, if $\gamma_{+}(A, K)$ is large, then $\gamma_{+}\left(A^{t}, K\right)$ is much smaller than $\gamma_{+}(A, K)$ for sufficiently large $t \in \mathbb{N}$. This is, in fact, not the case in general: in Section 9.2 we construct a metric space $\left(X, d_{X}\right)$ such that for each $n \in \mathbb{N}$ there is a symmetric stochastic matrix $A_{n}$ such that $\gamma_{+}\left(A_{n}, d_{X}^{2}\right) \geqslant n$ yet for every $t \in \mathbb{N}$ there is $n_{0} \in \mathbb{N}$ such that for all $n \geqslant n_{0}$ we have $\gamma_{+}\left(A_{n}^{t}, d_{X}^{2}\right) \gtrsim \gamma_{+}\left(A_{n}, d_{X}^{2}\right)$. The question which metric spaces satisfy the desired nonlinear spectral calculus inequality thus becomes a subtle issue which we believe is of fundamental importance, beyond the particular application that we present here. A large part of the present paper is devoted to addressing this question. We obtain rather satisfactory results which allow us to carry out a zigzag type construction of super-expanders, though we are still quite far from a complete understanding of the behavior of nonlinear spectral gaps under graph powers for non-Euclidean geometries.

1.3.3. Metric Markov cotype and spectral calculus. We will introduce a criterion for a metric space $\left(X, d_{X}\right)$, which is a bi-Lipschitz invariant, and prove that it implies that for every $n, m \in \mathbb{N}$ and every $n \times n$ symmetric stochastic matrix $A$ the Cesàro averages $\frac{1}{m} \sum_{t=0}^{m-1} A^{t}$ satisfy the following spectral calculus inequality.

$$
\gamma_{+}\left(\frac{1}{m} \sum_{t=0}^{m-1} A^{t}, d_{X}^{2}\right) \leqslant C(X) \max \left\{1, \frac{\gamma_{+}\left(A, d_{X}^{2}\right)}{m^{\varepsilon(X)}}\right\},
$$

where $C(X), \varepsilon(X) \in(0, \infty)$ depend only on the geometry of $X$ but not on $m, n$ and the matrix $A$. The fact that we can only prove such an inequality for Cesàro averages rather than powers does not create any difficulty in the ensuing argument, since Cesàro averages are compatible with iterative graph constructions based on the zigzag product.

Note that Cesàro averages have the following combinatorial interpretation in the case of graphs. Given an $n$-vertex $d$-regular graph $G=(V, E)$ let $\mathscr{A}_{m}(G)$ be the graph whose vertex set is $V$ and for every $t \in\{0, \ldots, m-1\}$ and $u, v \in V$ we draw $d^{m-1-t}$ edges joining $u, v$ for every walk in $G$ of length $t$ which starts at $u$ and terminates at $v$. With this definition $A_{\mathscr{A}_{m}(G)}=\frac{1}{m} \sum_{t=0}^{m-1} A_{G}^{t}$, and $\mathscr{A}_{m}(G)$ is $m d^{m-1}$-regular. We will slightly abuse this notation by also using the shorthand

$$
\mathscr{A}_{m}(A) \stackrel{\text { def }}{=} \frac{1}{m} \sum_{t=0}^{m-1} A^{t}
$$

when $A$ is an $n \times n$ matrix.

In the important paper [4 K. Ball introduced a linear property of Banach spaces that he called Markov cotype 2, and he indicated a two-step definition that could be used to extend this notion to general metric spaces. Motivated by Ball's ideas, we consider the following variant of his definition.

Definition 1.4 (Metric Markov cotype). Fix $p, q \in(0, \infty)$. A metric space $\left(X, d_{X}\right)$ has metric Markov cotype $p$ with exponent $q$ if there exists $C \in(0, \infty)$ such that for every $m, n \in \mathbb{N}$, every $n \times n$ symmetric stochastic matrix $A=\left(a_{i j}\right)$, and every $x_{1}, \ldots, x_{n} \in X$, there exist $y_{1}, \ldots, y_{n} \in X$ satisfying

$$
\sum_{i=1}^{n} d_{X}\left(x_{i}, y_{i}\right)^{q}+m^{q / p} \sum_{i=1}^{n} \sum_{j=1}^{n} a_{i j} d_{X}\left(y_{i}, y_{j}\right)^{q} \leqslant C^{q} \sum_{i=1}^{n} \sum_{j=1}^{n} \mathscr{A}_{m}(A)_{i j} d_{X}\left(x_{i}, x_{j}\right)^{q} .
$$


The infimum over those $C \in(0, \infty)$ for which (17) holds true is denoted $C_{p}^{(q)}\left(X, d_{X}\right)$. When $q=p$ we drop the explicit mention of the exponent and simply say that if (17) holds true with $q=p$ then $\left(X, d_{X}\right)$ has metric Markov cotype $p$.

Remark 1.5. We refer to [49, Sec. 4.1] for an explanation of the background and geometric intuition that motivates the (admittedly cumbersome) terminology of Definition 1.4. Briefly, the term "cotype" indicates that this definition is intended to serve as a metric analog of the important Banach space property Rademacher cotype (see [40]). Despite this fact, in the the forthcoming paper [45] we show, using a clever idea of Kalton [25], that there exists a Banach space with Rademacher cotype 2 that does not have metric Markov cotype $p$ for any $p \in(0, \infty)$. The term "Markov" in Definition 1.4 refers to the fact that the notion of metric Markov cotype is intended to serve as a certain "dual" to Ball's notion of Markov type [4], which is a notion which is defined in terms of the geometric behavior of stationary reversible Markov chains whose state space is a finite subset of $X$.

Remark 1.6. Ball's original definition [4] of metric Markov cotype is seemingly different from Definition [1.4, but in [45] we show that Definition 1.4 is equivalent to Ball's definition. We introduced Definition 1.4 since it directly implies Theorem 1.7 below.

The link between Definition 1.4 and the desired spectral calculus inequality (15) is contained in the following theorem, which is proved in Section 3 .

Theorem 1.7 (Metric Markov cotype implies nonlinear spectral calculus). Fix $p, C \in(0, \infty)$ and suppose that a metric space $\left(X, d_{X}\right)$ satisfies

$$
C_{p}^{(2)}\left(X, d_{X}\right) \leqslant C
$$

Then for every $m, n \in \mathbb{N}$, every $n \times n$ symmetric stochastic matrix $A$ satisfies

$$
\gamma_{+}\left(\mathscr{A}_{m}(A), d_{X}^{2}\right) \leqslant(45 C)^{2} \max \left\{1, \frac{\gamma_{+}\left(A, d_{X}^{2}\right)}{m^{2 / p}}\right\} .
$$

In Section 6.3 we investigate the metric Markov cotype of super-reflexive Banach spaces, obtaining the following result, whose proof is inspired by Ball's insights in [4].

Theorem 1.8 (Metric Markov cotype for super-reflexive Banach spaces). Let $\left(X,\|\cdot\|_{X}\right)$ be a super-reflexive Banach space. Then there exists $p=p(X) \in[2, \infty)$ such that

$$
C_{p}^{(2)}\left(X,\|\cdot\|_{X}\right)<\infty
$$

i.e., $\left(X,\|\cdot\|_{X}\right)$ has Metric Markov cotype $p$ with exponent 2.

Remark 1.9. In our forthcoming paper [45] we compute the metric Markov cotype of additional classes of metric spaces. In particular, we show that all $C A T(0)$ metric spaces (see [11]), and hence also all complete simply connected Riemannian manifolds with nonnegative sectional curvature, have Metric Markov cotype 2 with exponent 2.

By combining Theorem 1.7 and Theorem 1.8 we deduce the following result.

Corollary 1.10 (Nonlinear spectral calculus for super-reflexive Banach spaces). For every super-reflexive Banach space $\left(X,\|\cdot\|_{X}\right)$ there exist $\varepsilon(X), C(X) \in(0, \infty)$ such that for every $m, n \in \mathbb{N}$ and every $n \times n$ symmetric stochastic matrix $A$ we have

$$
\gamma_{+}\left(\mathscr{A}_{m}(A),\|\cdot\|_{X}^{2}\right) \leqslant C(X) \max \left\{1, \frac{\gamma_{+}\left(A,\|\cdot\|_{X}^{2}\right)}{m^{\varepsilon(X)}}\right\} .
$$


Remark 1.11. In Theorem 6.7 below we present a different approach to proving nonlinear spectral calculus inequalities in the setting of super-reflexive Banach spaces. This approach, which is based on bounding the norm of a certain linear operator, has the advantage that it establishes the decay of the Poincaré constant of the power $A^{m}$ rather than the Cesàro average $\mathscr{A}_{m}(A)$. While this result is of independent geometric interest, the form of the decay inequality that we are able to obtain has the disadvantage that we do not see how to use it to construct super-expanders. Moreover, we do not know how to obtain submultiplicativity estimates for such norm bounds under zigzag products and other graph products such as the tensor product and replacement product (see Section 1.3.5 below). The approach based on metric Markov cotype also has the advantage of being applicable to other classes of (non-Banach) metric spaces, in addition to its usefulness for the Lipschitz extension problem [4, 45].

1.3.4. The base graph. In order to construct super-expanders using Theorem 1.3 and Corollary 1.10 one must start the inductive procedure with an appropriate "base graph". This is a nontrivial issue that raises analytic challenges which are interesting in their own right.

It is most natural to perform our construction of base graphs in the context of $K$-convex Banach spaces, which, as we recalled earlier, is a class of spaces that is strictly larger than the class of super-reflexive spaces. The result thus obtained, proved in Section 7 using the preparatory work in Section 5.2 and part of Section [6, reads as follows.

Lemma 1.12 (Existence of base graphs for $K$-convex spaces). There exists a strictly increasing sequence of integers $\left\{m_{n}\right\}_{n=1}^{\infty} \subseteq \mathbb{N}$ satisfying

$$
\forall n \in \mathbb{N}, \quad 2^{n / 10} \leqslant m_{n} \leqslant 2^{n},
$$

with the following properties. For every $\delta \in(0,1]$ there is $n_{0}(\delta) \in \mathbb{N}$ and a sequence of regular graphs $\left\{H_{n}(\delta)\right\}_{n=n_{0}(\delta)}^{\infty}$ such that

- $\left|V\left(H_{n}(\delta)\right)\right|=m_{n}$ for every integer $n \geqslant n_{0}(\delta)$.

- For every $n \in\left[n_{0}(\delta), \infty\right) \cap \mathbb{N}$ the degree of $H_{n}(\delta)$, denoted $d_{n}(\delta)$, satisfies

$$
d_{n}(\delta) \leqslant e^{\left(\log m_{n}\right)^{1-\delta}} .
$$

- For every $K$-convex Banach space $\left(X,\|\cdot\|_{X}\right)$ we have $\gamma_{+}\left(H_{n}(\delta),\|\cdot\|_{X}^{2}\right)<\infty$ for all $\delta \in(0,1)$ and $n \in \mathbb{N} \cap\left[n_{0}(\delta), \infty\right)$. Moreover, there exists $\delta_{0}(X) \in(0,1)$ such that

$$
\forall \delta \in\left(0, \delta_{0}(X)\right], \forall n \in\left[n_{0}(\delta), \infty\right) \cap \mathbb{N}, \quad \gamma_{+}\left(H_{n}(\delta),\|\cdot\|_{X}^{2}\right) \leqslant 9^{3} .
$$

The bound $9^{3}$ in (20) is nothing more than an artifact of our proof and it does not play a special role in what follows: all that we will need for the purpose of constructing superexpanders is to ensure that

$$
\sup _{\delta \in\left(0, \delta_{0}(X)\right]} \sup _{n \in\left[n_{0}(\delta), \infty\right) \cap \mathbb{N}} \gamma_{+}\left(H_{n}(\delta),\|\cdot\|_{X}^{2}\right)<\infty,
$$

i.e., for our purposes the upper bound on $\gamma_{+}\left(H_{n}(\delta),\|\cdot\|_{X}^{2}\right)$ can be allowed to depend on $X$. Moreover, in the ensuing arguments we can make do with a degree bound that is weaker than (19): all we need is that

$$
\forall \delta \in(0,1), \quad \lim _{n \rightarrow \infty} \frac{\log d_{n}(\delta)}{\log m_{n}}=0 .
$$


However, we do not see how to prove the weaker requirements (21), (22) in a substantially simpler way than our proof of the stronger requirements (19), (20).

The starting point of our approach to construct base graphs is the "hypercube quotient argument" of [27], although in order to apply such ideas in our context we significantly modify this construction, and apply deep methods of Pisier [58, 59]. A key analytic challenge that arises here is to bound the norm of the inverse of the hypercube Laplacian on the vectorvalued tail space, i.e., the space of all functions taking values in a Banach space $X$ whose Fourier expansion is supported on Walsh functions corresponding to large sets. If $X$ is a Hilbert space then the desired estimate is an immediate consequence of orthogonality, but even when $X$ is an $L_{p}(\mu)$ space the corresponding inequalities are not known. P.-A. Meyer [46] previously obtained $L_{p}$ bounds for the inverse of the Laplacian on the (real-valued) tail space, but such bounds are insufficient for our purposes. In order to overcome this difficulty, in Section 5 we obtain decay estimates for the heat semigroup on the tail space of functions taking values in a $K$-convex Banach space. We then use (in Section 7) the heat semigroup to construct a new (more complicated) hypercube quotient by a linear code which can serve as the base graph of Lemma 1.12 .

The bounds on the norm of the heat semigroup on the vector valued tail space (and the corresponding bounds on the norm of the inverse of the Laplacian) that are proved in Section 5 are sufficient for the purpose of proving Lemma 1.12, but we conjecture that they are suboptimal. Section 5 contains analytic questions along these lines whose positive solution would yield a simplification of our construction of the base graph (see Remark 7.5).

With all the ingredients in place (Theorem 1.3, Corollary 1.10, Lemma 1.12), the actual iterative construction of super-expanders in performed in Section 4 . Since we need to construct a single sequence of bounded degree graphs that has a nonlinear spectral gap with respect to all super-reflexive Banach spaces, our implementation of the zigzag strategy is significantly more involved than the zigzag iteration of Reingold, Vadhan and Wigderson (recall Section 1.3.1). This implementation itself may be of independent interest.

1.3.5. Sub-multiplicativity theorems for graph products. Theorem 1.3 is a special case of a a larger family of sub-multiplicativity estimates for nonlinear spectral gaps with respect to certain graph products. The literature contains several combinatorial procedures to combine two graphs, and it turns out that such constructions are often highly compatible with nonlinear Poincaré inequalities. In Section 8 we further investigate this theme.

The main results of Section 8 are collected in the following theorem (the relevant terminology is discussed immediately after its statement). Item (III) below is nothing more than a restatement of Theorem 1.3 .

Theorem 1.13. Fix $m, n, n_{1}, d_{1}, d_{2} \in \mathbb{N}$. Suppose that $K: X \times X \rightarrow[0, \infty)$ is a kernel and $\left(Y, d_{Y}\right)$ is a metric space. Suppose also that $G_{1}=\left(V_{1}, E_{1}\right)$ is a $d_{1}$-regular graph with $n_{1}$ vertices and $G_{2}=\left(V_{2}, E_{2}\right)$ is a $d_{2}$-regular graph with $d_{1}$ vertices. Then,

(I) If $A=\left(a_{i j}\right)$ is an $m \times m$ symmetric stochastic matrix and $B=\left(b_{i j}\right)$ is an $n \times n$ symmetric stochastic matrix then the tensor product $A \otimes B$ satisfies

$$
\gamma_{+}(A \otimes B, K) \leqslant \gamma_{+}(A, K) \cdot \gamma_{+}(B, K) .
$$

(II) The zigzag product $G_{1}$ (2) $G_{2}$ satisfies

$$
\gamma_{+}\left(G_{1}(\mathrm{Z}) G_{2}, K\right) \leqslant \gamma_{+}\left(G_{1}, K\right) \cdot \gamma_{+}\left(G_{2}, K\right)^{2} .
$$


(III) The derandomized square $G_{1}\left(G_{2}\right.$ satisfies

$$
\gamma_{+}\left(G_{1} \subseteq G_{2}, K\right) \leqslant \gamma_{+}\left(G_{1}^{2}, K\right) \cdot \gamma_{+}\left(G_{2}, K\right) .
$$

(IV) The replacement product $G_{1} \odot G_{2}$ satisfies

$$
\gamma_{+}\left(G_{1} \odot G_{2}, d_{Y}^{2}\right) \leqslant 3\left(d_{2}+1\right) \cdot \gamma_{+}\left(G_{1}, d_{Y}^{2}\right) \cdot \gamma_{+}\left(G_{2}, d_{Y}^{2}\right)^{2} .
$$

(V) The balanced replacement product $G_{1}$ (b) $G_{2}$ satisfies

$$
\gamma_{+}\left(G_{1} \text { (b) } G_{2}, d_{Y}^{2}\right) \leqslant 6 \cdot \gamma_{+}\left(G_{1}, d_{Y}^{2}\right) \cdot \gamma_{+}\left(G_{2}, d_{Y}^{2}\right)^{2} .
$$

Since the $(m n) \times(m n)$ matrix $A \otimes B=\left(a_{i j} b_{k \ell}\right)$ satisfies $\lambda(A \otimes B)=\max \{\lambda(A), \lambda(B)\}$, in the Euclidean case, i.e., $K: \mathbb{R} \times \mathbb{R} \rightarrow[0, \infty)$ is given by $K(x, y)=(x-y)^{2}$, the product in the right hand side of (23) can be replaced by a maximum. Lemma 8.2 below contains a similar improvement of (23) under additional assumptions on the kernel $K$.

The definitions of the graph products $G_{1}$ (2) $G_{2}, G_{1}\left(G_{2}, G_{1}(\mathrm{P}) G_{2}, G_{1}\right.$ (b) $G_{2}$ are recalled in Section 8 . The replacement product $\left.G_{1} \mathrm{P}\right) G_{2}$, which is a $\left(d_{2}+1\right)$-regular graph with $n_{1} d_{1}$ vertices, was introduced by Gromov in [17, where he applied it iteratively to hypercubes of logarithmically decreasing size so as to obtain a constant degree graph which has sufficiently good expansion for his (geometric) application. In [17] Gromov bounded $\lambda\left(G_{1} \odot G_{2}\right)$ from above by an expression involving only $\lambda\left(G_{1}\right), \lambda\left(G_{2}\right), d_{2}$. Such a bound was also obtained by Reingold, Vadhan and Wigderson in [64. We shall use (26) in the proof of Theorem 1.1.

The breakthrough of Reingold, Vadhan and Wigderson [64] introduced the zigzag product, which can be used to construct constant degree expanders; the fact that (24) holds true for general kernels $K$, while (26) assumes that $d_{Y}$ is a metric and incurs a multiplicative loss of $3\left(d_{2}+1\right)$ can be viewed as an indication why the zigzag product is a more basic operation than the replacement product.

The balanced replacement product $G_{1}$ (b) $G_{2}$, which is a $2 d_{2}$-regular graph with $n_{1} d_{1}$ vertices, was introduced by Reingold, Vadhan and Wigderson [64], who bounded $\lambda\left(G_{1}(\mathrm{~b}) G_{2}\right)$ from above by an expression involving only $\lambda\left(G_{1}\right), \lambda\left(G_{2}\right)$.

The derandomized square $G_{1}\left(G_{2}\right.$, which is a $d_{1} d_{2}$-regular graph with $n_{1}$ vertices, was introduced by Rozenman and Vadhan in [66], where they bounded $\lambda\left(G_{1} @ G_{2}\right)$ from above by an expression involving only $\lambda\left(G_{1}\right), \lambda\left(G_{2}\right)$. This operation is of a different nature: it aims to create a graph that has spectral properties similar to the square $G_{1}^{2}$, but with significantly fewer edges. In 64, 66 tensor products and derandomized squaring were used to improve the computational efficiency of zigzag constructions. The general bounds (23) and (25) can be used to improve the efficiency of our constructions in a similar manner, but we will not explicitly discuss computational efficiency issues in this paper (this, however, is relevant to our forthcoming paper [44, where our construction is used for an algorithmic purpose).

\section{Preliminary Results on nOnlinear sPeCtral GaPS}

The purpose of this section is to record some simple and elementary preliminary facts about nonlinear spectral gaps that will be used throughout this article. One can skip this section on first reading and refer back to it only when the facts presented here are used in the subsequent sections. 
2.1. The trivial bound for general graphs. For $\kappa \in[0, \infty)$ a kernel $\rho: X \times X \rightarrow[0, \infty)$ is called a $2^{\kappa}$-quasi-semimetric if for every $x, y, z \in X$ we have

$$
\rho(x, y) \leqslant 2^{\kappa}(\rho(x, z)+\rho(z, y)) .
$$

The key examples of $2^{\kappa}$-quasi-semimetrics are of the form $\rho=d_{X}^{p}$, where $d_{X}: X \times X \rightarrow[0, \infty)$ is a semimetric and $p \in[1, \infty)$, in which case $\kappa=p-1$.

Lemma 2.1. Fix $n, d \in \mathbb{N}$ and $\kappa \in[0, \infty)$. Let $G=(V, E)$ be a d-regular connected graph with $n$ vertices. Then for every $2^{\kappa}$-quasi-semimetric $\rho: X \times X \rightarrow[0, \infty)$ we have

$$
\gamma(G, \rho) \leqslant 2^{\kappa-1} d n^{\kappa+1} .
$$

If in addition $G$ is not a bipartite graph then

$$
\gamma_{+}(G, \rho) \leqslant 2^{2 \kappa} d n^{\kappa+1} \text {. }
$$

Proof. For every $x, y \in V$ choose distinct $\left\{u_{0}^{x, y}=x, u_{1}^{x, y}, \ldots, u_{m_{x, y}-1}^{x, y}, u_{m_{x, y}}^{x, y}=y\right\} \subseteq V$ such that $\left(u_{i}^{x, y}, u_{i-1}^{x, y}\right) \in E$ for every $i \in\left\{1, \ldots, m_{x, y}\right\}$, and $\left(u_{i}^{x, y}, u_{i-1}^{x, y}\right) \neq\left(u_{j}^{x, y}, u_{j-1}^{x, y}\right)$ for distinct $i, j \in\left\{1, \ldots, m_{x, y}\right\}$. Fixing $f: V \rightarrow X$, a straightforward inductive application of (28) yields

$$
\rho(f(x), f(y)) \leqslant\left(2 m_{x, y}\right)^{\kappa} \sum_{i=1}^{m_{x, y}} \rho\left(f\left(u_{i-1}^{x, y}\right), f\left(u_{i}^{x, y}\right)\right) \leqslant(2 n)^{\kappa} \sum_{i=1}^{m_{x, y}} \rho\left(f\left(u_{i-1}^{x, y}\right), f\left(u_{i}^{x, y}\right)\right) .
$$

Thus

$$
\begin{aligned}
\frac{1}{n^{2}} \sum_{(x, y) \in V \times V} \rho(f(x), f(y)) & \leqslant \frac{(2 n)^{\kappa}}{n^{2}} \sum_{(x, y) \in V \times V} \sum_{i=1}^{m_{x, y}} \rho\left(f\left(u_{i-1}^{x, y}\right), f\left(u_{i}^{x, y}\right)\right) \\
& \leqslant \frac{(2 n)^{\kappa}\left(\begin{array}{l}
n \\
2
\end{array}\right)}{n^{2}} \sum_{(a, b) \in E} \rho(f(a), f(b)) \leqslant \frac{(2 n)^{\kappa} n d}{2} \cdot \frac{1}{n d} \sum_{(a, b) \in E} \rho(f(a), f(b)) .
\end{aligned}
$$

This proves (29). To prove (30) suppose that $G$ is connected but not bipartite. Then for every $x, y \in V$ there exists a path of odd length joining $x$ and $y$ whose total length is at most $2 n$ and in which each edge is repeated at most once (indeed, being non-bipartite, $G$ contains an odd cycle $c$; the desired path can be found by considering the shortest paths joining $x$ and $y$ with $c$ ). Let $\left\{w_{0}^{x, y}=x, w_{1}^{x, y}, \ldots, w_{m-1}^{x, y}, w_{2 \ell_{x, y}+1}^{x, y}=y\right\} \subseteq V$ be such a path. For every $f, g: V \rightarrow X$ we have

$$
\begin{aligned}
\sum_{(x, y) \in V \times V} \rho(f(x), g(y)) \leqslant & \sum_{(x, y) \in V \times V}\left(4 \ell_{x, y}+2\right)^{\kappa}\left(\rho\left(f\left(w_{0}^{x, y}\right), g\left(w_{1}^{x, y}\right)\right)\right. \\
& \left.\quad+\sum_{i=1}^{\ell x, y}\left(\rho\left(g\left(w_{2 i-1}^{x, y}\right), f\left(w_{2 i}^{x, y}\right)\right)+\rho\left(f\left(w_{2 i}^{x, y}\right), g\left(w_{2 i+1}^{x, y}\right)\right)\right)\right) \\
\leqslant & (4 n)^{\kappa} \cdot n^{2} \sum_{(a, b) \in E} \rho(f(a), g(b)),
\end{aligned}
$$

implying (30). 
Remark 2.2. For $n \in \mathbb{N}$ let $C_{n}$ denote the $n$-cycle and let $C_{n}^{\circ}$ denote the $n$-cycle with self loops (thus $C_{n}^{\circ}$ is a 3-regular graph). It follows from Lemma 2.1 that $\gamma\left(C_{n}, \rho\right) \lesssim(2 n)^{\kappa+1}$ and $\gamma_{+}\left(C_{n}^{\circ}, \rho\right) \lesssim(4 n)^{\kappa+1}$ for every $2^{\kappa}$-quasi-semimetric. If $\left(X, d_{X}\right)$ is a metric space and $p \in[1, \infty)$ then one can refine the above arguments using the symmetry of the circle to get the improved bound

$$
\gamma_{+}\left(C_{n}^{\circ}, d_{X}^{p}\right) \lesssim \frac{(n+1)^{p}}{p 2^{p}}
$$

We omit the proof of (31) since the improved dependence on $p$ is not used in the ensuing discussion.

2.2. $\gamma$ versus $\gamma_{+}$. By taking $f=g$ in the definition of $\gamma_{+}(\cdot, \cdot)$ one immediately sees that $\gamma(A, K) \leqslant \gamma_{+}(A, K)$ for every kernel $K: X \times X \rightarrow[0, \infty)$ and every symmetric stochastic matrix $A$. Here we investigate additional relations between these quantities.

Lemma 2.3. Fix $\kappa \in[0, \infty)$ and let $\rho: X \times X \rightarrow[0, \infty)$ be a $2^{\kappa}$-quasi-semimetric. Then for every symmetric stochastic matrix $A$ we have

$$
\frac{2}{2^{\kappa+1}+1} \gamma\left(\left(\begin{array}{cc}
0 & A \\
A & 0
\end{array}\right), \rho\right) \leqslant \gamma_{+}(A, \rho) \leqslant 2 \gamma\left(\left(\begin{array}{ll}
0 & A \\
A & 0
\end{array}\right), \rho\right)
$$

Proof. Fix $f, g:\{1, \ldots, n\} \rightarrow X$ and define $h:\{1, \ldots, 2 n\} \rightarrow X$ by

$$
h(i) \stackrel{\text { def }}{=} \begin{cases}f(i) & \text { if } i \in\{1, \ldots, n\}, \\ g(i-n) & \text { if } i \in\{n+1, \ldots, 2 n\} .\end{cases}
$$

Suppose that $A=\left(a_{i j}\right)$ is an $n \times n$ symmetric stochastic matrix. Then

$$
\begin{aligned}
& \frac{1}{n^{2}} \sum_{i=1}^{n} \sum_{j=1}^{n} \rho(f(i), g(j))=\frac{1}{n^{2}} \sum_{i=1}^{n} \sum_{j=1}^{n} \rho(h(i), h(j+n)) \leqslant \frac{1}{2 n^{2}} \sum_{i=1}^{2 n} \sum_{j=1}^{2 n} \rho(h(i), h(j)) \\
& \leqslant \frac{2 \gamma\left(\left(\begin{array}{cc}
0 & A \\
A & 0
\end{array}\right), \rho\right)}{2 n} \sum_{i=1}^{2 n} \sum_{j=1}^{2 n}\left(\begin{array}{cc}
0 & A \\
A & 0
\end{array}\right)_{i j} \rho(h(i), h(j))=\frac{2 \gamma\left(\left(\begin{array}{cc}
0 & A \\
A & 0
\end{array}\right), \rho\right)}{n} \sum_{i=1}^{n} \sum_{j=1}^{n} a_{i j} \rho(f(i), f(j)) .
\end{aligned}
$$

This proves the rightmost inequality in (32). Note that for this inequality the quasimetric inequality (28) was not used, and therefore $\rho$ can be an arbitrarily kernel.

To prove the leftmost inequality in (32) we argue as follows. Fix $h:\{1, \ldots, 2 n\} \rightarrow X$ and define $f, g:\{1, \ldots, n\} \rightarrow X$ by $f(i)=h(i)$ and $g(i)=h(i+n)$ for every $i \in\{1, \ldots, n\}$. Then

$$
\begin{aligned}
\sum_{i=1}^{n} \sum_{j=1}^{n} \rho(h(i), h(j)) & \leqslant \frac{1}{n} \sum_{i=1}^{n} \sum_{j=1}^{n} \sum_{\ell=1}^{n} 2^{\kappa}(\rho(h(i), h(\ell+n))+\rho(h(j), h(\ell+n))) \\
& =2^{\kappa+1} \sum_{i=1}^{n} \sum_{j=1}^{n} \rho(f(i), g(j)) .
\end{aligned}
$$


Similarly,

$$
\begin{aligned}
\sum_{i=1}^{n} \sum_{j=1}^{n} \rho(h(i+n), h(j+n)) & \leqslant \frac{1}{n} \sum_{i=1}^{n} \sum_{j=1}^{n} \sum_{\ell=1}^{n} 2^{\kappa}(\rho(h(i+n), h(\ell))+\rho(h(j+n), h(\ell))) \\
& =2^{\kappa+1} \sum_{i=1}^{n} \sum_{j=1}^{n} \rho(f(i), g(j))
\end{aligned}
$$

Hence,

$$
\begin{aligned}
& \frac{1}{(2 n)^{2}} \sum_{i=1}^{2 n} \sum_{j=1}^{2 n} \rho(h(i), h(j)) \\
& =\frac{1}{(2 n)^{2}} \sum_{i=1}^{n} \sum_{j=1}^{n} \rho(h(i), h(j))+\frac{1}{(2 n)^{2}} \sum_{i=1}^{n} \sum_{j=1}^{n} \rho(h(i+n), h(j+n)) \\
& +\frac{1}{(2 n)^{2}} \sum_{i=1}^{n} \sum_{j=1}^{n} \rho(h(i), h(j+n))+\frac{1}{(2 n)^{2}} \sum_{i=1}^{n} \sum_{j=1}^{n} \rho(h(i+n), h(j)) \\
& \stackrel{(33) \wedge(34)}{\leqslant} \frac{2^{\kappa+1}+1}{2 n^{2}} \sum_{i=1}^{n} \sum_{j=1}^{n} \rho(f(i), g(j)) \\
& \leqslant \quad \frac{\left(2^{\kappa+1}+1\right) \gamma_{+}(A, \rho)}{2 n} \sum_{i=1}^{n} \sum_{j=1}^{n} a_{i j} \rho(f(i), g(j)) \\
& =\frac{\left(2^{\kappa+1}+1\right) \gamma_{+}(A, \rho)}{2} \cdot \frac{1}{2 n} \sum_{i=1}^{2 n} \sum_{j=1}^{2 n}\left(\begin{array}{cc}
0 & A \\
A & 0
\end{array}\right)_{i j} \rho(h(i), h(j)),
\end{aligned}
$$

which is precisely the leftmost inequality in (32).

Lemma 2.4. Fix $\kappa \in[0, \infty)$ and let $\rho: X \times X \rightarrow[0, \infty)$ be a $2^{\kappa}$-quasi-semimetric. Then for every symmetric stochastic matrix $A$ we have

$$
\gamma\left(\left(\begin{array}{cc}
0 & \mathscr{A}_{m}(A) \\
\mathscr{A}_{m}(A) & 0
\end{array}\right), \rho\right) \leqslant\left(2^{\kappa+2}+1\right) \gamma\left(\mathscr{A}_{m}\left(\begin{array}{cc}
0 & A \\
A & 0
\end{array}\right), \rho\right) .
$$

Proof. Suppose that $A=\left(a_{i j}\right)$ is an $n \times n$ symmetric stochastic matrix. It suffices to show that for every $h:\{1, \ldots, 2 n\} \rightarrow X$ and every $m \in \mathbb{N}$ we have

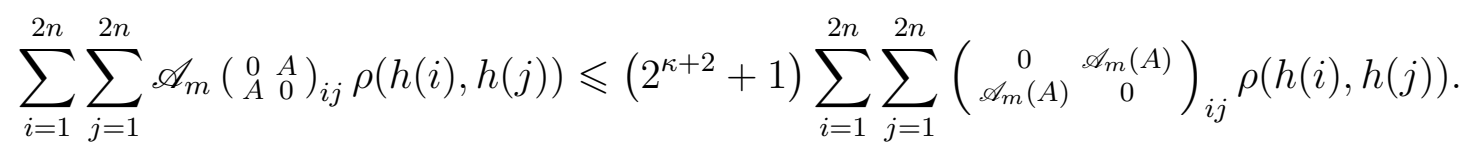

For simplicity of notation write $B=\left(b_{i j}\right) \stackrel{\text { def }}{=}\left(\begin{array}{cc}0 & A \\ A & 0\end{array}\right)$. Then

$$
\mathscr{A}_{m}(B)=\frac{1}{m} I+\frac{1}{m} \sum_{s=1}^{\lfloor(m-1) / 4\rfloor} B^{4 s}+\frac{1}{m} \sum_{s=0}^{\lfloor(m-3) / 4\rfloor} B^{2(2 s+1)}+\frac{1}{m} \sum_{s=0}^{\lfloor(m-2) / 2\rfloor} B^{2 s+1} .
$$

Observe that

$$
t \in 2 \mathbb{N}-1 \Longrightarrow\left(\begin{array}{ll}
0 & A \\
A & 0
\end{array}\right)^{t}=\left(\begin{array}{cc}
0 & A^{t} \\
A^{t} & 0
\end{array}\right)
$$


Hence,

$$
\frac{1}{m} \sum_{s=0}^{\lfloor(m-2) / 2\rfloor} B^{2 s+1}=\left(\begin{array}{cc}
0 & \frac{1}{m} \sum_{s=0}^{\lfloor(m-2) / 2\rfloor} A^{2 s+1} \\
\frac{1}{m} \sum_{s=0}^{\lfloor(m-2) / 2\rfloor} A^{2 s+1} & 0
\end{array}\right) .
$$

For every $s \in \mathbb{N}$, using the fact that $B^{2 s-1}$ and $B^{2 s+1}$ are symmetric and stochastic, we have

$$
\begin{aligned}
& \sum_{i=1}^{2 n} \sum_{j=1}^{2 n}\left(B^{4 s}\right)_{i j} \rho(h(i), h(j)) \\
& \quad \leqslant \sum_{i=1}^{2 n} \sum_{j=1}^{2 n}\left(\sum_{\ell=1}^{2 n}\left(B^{2 s-1}\right)_{i \ell}\left(B^{2 s+1}\right)_{\ell j} 2^{\kappa}(\rho(h(i), h(\ell))+\rho(h(\ell), h(j)))\right) \\
& \quad=2^{\kappa} \sum_{a=1}^{2 n} \sum_{b=1}^{2 n}\left(\begin{array}{c}
0 \\
A^{2 s-1}+A^{2 s+1}
\end{array} \begin{array}{c}
A^{2 s-1}+A^{2 s+1} \\
0
\end{array}\right)_{a b} \rho(h(a), h(b)) .
\end{aligned}
$$

Similarly, for every $s \in \mathbb{N} \cup\{0\}$,

$$
\sum_{i=1}^{2 n} \sum_{j=1}^{2 n}\left(B^{2(2 s+1)}\right)_{i j} \rho(h(i), h(j)) \leqslant 2^{\kappa+1} \sum_{a=1}^{2 n} \sum_{b=1}^{2 n}\left(\begin{array}{cc}
0 & A^{2 s+1} \\
A^{2 s+1} & 0
\end{array}\right)_{a b} \rho(h(a), h(b)) .
$$

It follows from (37), (38), (39) and (40) that

$$
\sum_{i=1}^{2 n} \sum_{j=1}^{2 n} \mathscr{A}_{m}(B) \rho(h(i), h(j)) \leqslant \sum_{i=1}^{2 n} \sum_{j=1}^{2 n}\left(\begin{array}{cc}
0 & C \\
C & 0
\end{array}\right)_{i j} \rho(h(i), h(j))
$$

where

$$
C \stackrel{\text { def }}{=} \frac{1}{m} I+\frac{2^{\kappa}}{m} \sum_{s=1}^{\lfloor(m-1) / 4\rfloor}\left(A^{2 s-1}+A^{2 s+1}\right)+\frac{2^{\kappa+1}}{m} \sum_{s=0}^{\lfloor(m-3) / 4\rfloor} A^{2 s+1}+\frac{1}{m} \sum_{s=0}^{\lfloor(m-2) / 2\rfloor} A^{2 s+1} .
$$

To deduce (36) from (41) it remains to observe that

$$
\forall i, j \in\{1, \ldots, n\}, \quad C_{i j} \leqslant\left(2^{\kappa+2}+1\right) \mathscr{A}_{m}(A)_{i j}
$$

The following two lemmas are intended to indicate that if one is only interested in the existence of super-expanders (rather than estimating the nonlinear spectral gap of a specific graph of interest) then the distinction between $\gamma(\cdot, \cdot)$ and $\gamma_{+}(\cdot, \cdot)$ is not very significant.

Lemma 2.5. Fix $n, d \in \mathbb{N}$ and let $G=(V, W, E)$ be a d-regular bipartite graph such that $|V|=|W|=n$. Then there exists a 2d-regular graph $H=(V, F)$ for which every kernel $K: X \times X \rightarrow[0, \infty)$ satisfies $\gamma_{+}(H, K) \leqslant 2 \gamma(G, K)$.

Proof. Fix an arbitrary bijection $\sigma: V \rightarrow W$. The new edges $F$ on the vertex set $V$ are given by

$$
\forall(u, v) \in V \times V, \quad F(u, v) \stackrel{\text { def }}{=} E(u, \sigma(v))+E(\sigma(u), v) .
$$

Thus $(V, F)$ is a $2 d$-regular graph.

Given $f, g: V \rightarrow X$ define $\phi_{1}, \phi_{2}: V \cup W \rightarrow X$ by

$$
\phi_{1}(x) \stackrel{\text { def }}{=}\left\{\begin{array} { l l } 
{ f ( x ) } & { \text { if } x \in V , } \\
{ g ( \sigma ^ { - 1 } ( x ) ) } & { \text { if } x \in W , }
\end{array} \quad \text { and } \quad \phi _ { 2 } ( x ) \stackrel { \text { def } } { = } \left\{\begin{array}{ll}
g(x) & \text { if } x \in V, \\
f\left(\sigma^{-1}(x)\right) & \text { if } x \in W .
\end{array}\right.\right.
$$


Then,

$$
\begin{aligned}
& \frac{1}{n^{2}} \sum_{(u, v) \in V \times V} K(f(u), g(v)) \\
& \leqslant \frac{1}{(2 n)^{2}} \sum_{(x, y) \in(V \cup W) \times(V \cup W)}\left(K\left(\phi_{1}(x), \phi_{1}(y)\right)+K\left(\phi_{2}(x), \phi_{2}(y)\right)\right) \\
& \leqslant \frac{\gamma(G, K)}{2 n d} \sum_{(x, y) \in(V \times W) \cup(W \times V)} E(x, y)\left(K\left(\phi_{1}(x), \phi_{1}(y)\right)+K\left(\phi_{2}(x), \phi_{2}(y)\right)\right) \\
& =\frac{\gamma(G, K)}{n d} \sum_{(u, v) \in V \times V}(E(u, \sigma(v))+E(\sigma(u), v)) K(f(u), g(v)) \\
& =\frac{2 \gamma(G, K)}{n \cdot(2 d)} \sum_{(u, v) \in F} K(f(u), g(v)) .
\end{aligned}
$$

Lemma 2.6. Fix $n, d \in \mathbb{N}$ and let $G=(V, E)$ be a d-regular graph with $|V|=2 n$. Then there exists a $4 d$-regular graph $G^{\prime}=\left(V^{\prime}, E^{\prime}\right)$ with $\left|V^{\prime}\right|=n$ such that for every $\kappa \in(0, \infty)$ and every $\rho: X \times X \rightarrow[0, \infty)$ which is a $2^{\kappa}$-quasi-semimetric we have $\gamma_{+}\left(G^{\prime}, \rho\right) \leqslant 2^{\kappa+2} \gamma(G, \rho)$.

Proof. Write $V=V^{\prime} \cup V^{\prime \prime}$, where $V^{\prime}, V^{\prime \prime} \subseteq V$ are disjoint subsets of cardinality $n$, and fix an arbitrary bijection $\sigma: V^{\prime} \rightarrow V^{\prime \prime}$. We first define a bipartite graph $H=\left(V^{\prime}, V^{\prime \prime}, F\right)$ by

$$
\forall(x, y) \in V^{\prime} \times V^{\prime \prime}, \quad F(x, y) \stackrel{\text { def }}{=} E(x, y)+E\left(x, \sigma^{-1}(y)\right)+d \mathbf{1}_{\{y=\sigma(x)\}},
$$

where $F$ is extended to $V^{\prime \prime} \times V^{\prime}$ by imposing symmetry. This makes $H$ be a $2 d$-regular bipartite graph. We shall now estimate $\gamma(H, \rho)$. For every $f: V \rightarrow X$ we have

$$
\begin{aligned}
\frac{1}{(2 n)^{2}} \sum_{(u, v) \in V \times V} \rho(f(u), f(v)) \leqslant \frac{\gamma(G, \rho)}{2 n d}\left(\sum_{(u, v) \in\left(V^{\prime} \times V^{\prime \prime}\right) \cup\left(V^{\prime \prime} \times V^{\prime}\right)} E(u, v) \rho(f(u), f(v))\right. \\
\left.\quad+\sum_{(u, v) \in V^{\prime} \times V^{\prime}} E(u, v) \rho(f(u), f(v))+\sum_{(u, v) \in V^{\prime \prime} \times V^{\prime \prime}} E(u, v) \rho(f(u), f(v))\right) .
\end{aligned}
$$

Now, using the fact that $\rho$ is a $2^{\kappa}$-quasi-semimetric we have

$$
\begin{gathered}
\sum_{(u, v) \in V^{\prime} \times V^{\prime}} E(u, v) \rho(f(u), f(v)) \leqslant \sum_{(u, v) \in V^{\prime} \times V^{\prime}} 2^{\kappa} E(u, v)(\rho(f(u), f(\sigma(v)))+\rho(f(\sigma(v)), f(v))) \\
=2^{\kappa} \sum_{(x, y) \in V^{\prime} \times V^{\prime \prime}} E\left(x, \sigma^{-1}(y)\right) \rho(f(x), f(y))+2^{\kappa} d \sum_{z \in V^{\prime}} \rho(f(\sigma(z)), f(z)) .
\end{gathered}
$$

Similarly,

$$
\begin{aligned}
\sum_{(u, v) \in V^{\prime \prime} \times V^{\prime \prime}} E(u, v) & \rho(f(u), f(v)) \\
& \leqslant 2^{\kappa} \sum_{(x, y) \in V^{\prime \prime} \times V^{\prime}} E(x, \sigma(y)) \rho(f(x), f(y))+2^{\kappa} d \sum_{z \in V^{\prime}} \rho(f(z), f(\sigma(z))) .
\end{aligned}
$$


Recalling (42), we conclude from (43), (44) and (45) that

$$
\frac{1}{(2 n)^{2}} \sum_{(u, v) \in\left(V^{\prime} \cup V^{\prime \prime}\right) \times\left(V^{\prime} \cup V^{\prime \prime}\right)} \rho(f(u), f(v)) \leqslant \frac{2^{\kappa+1} \gamma(G, \rho)}{(2 n) \cdot(2 d)} \sum_{(x, y) \in F} \rho(f(x), f(y)) .
$$

Hence $\gamma(H, \rho) \leqslant 2^{\kappa+1} \gamma(G, \rho)$. The desired assertion now follows from Lemma 2.5,

2.3. Edge completion. In the ensuing arguments we will sometimes add edges to a graph in order to ensure that it has certain desirable properties, but we will at the same time want to control the Poincaré constants of the resulting denser graph. The following very easy facts will be useful for this purpose.

Lemma 2.7. Fix $n, d_{1}, d_{2} \in \mathbb{N}$. Let $G_{1}=\left(V, E_{1}\right)$ and $G_{2}=\left(V, E_{2}\right)$ be two n-vertex graphs on the same vertex set with $E_{2} \supseteq E_{1}$. Suppose that $G_{1}$ is $d_{1}$-regular and $G_{2}$ is $d_{2}$-regular. Then for every kernel $K: X \times X \rightarrow[0, \infty)$ we have

$$
\max \left\{\frac{\gamma\left(G_{2}, K\right)}{\gamma\left(G_{1}, K\right)}, \frac{\gamma_{+}\left(G_{2}, K\right)}{\gamma_{+}\left(G_{1}, K\right)}\right\} \leqslant \frac{d_{2}}{d_{1}}
$$

Proof. One just has to note that for every $f, g: V \rightarrow X$ we have

$$
\frac{1}{n d_{2}} \sum_{(x, y) \in E_{2}} K(f(x), g(y)) \geqslant \frac{1}{n d_{2}} \sum_{(x, y) \in E_{1}} K(f(x), g(y))=\frac{d_{1}}{d_{2}} \cdot \frac{1}{n d_{1}} \sum_{(x, y) \in E_{1}} K(f(x), g(y)) .
$$

Definition 2.8 (Edge completion). Fix two integers $D \geqslant d \geqslant 2$. Let $G=(V, E)$ be a $d$-regular graph. The $D$-edge completion of $G$, denoted $\mathscr{C}_{D}(G)$, is defined as a graph on the same vertex set $V$, with edges $E\left(\mathscr{C}_{D}(G)\right) \supseteq E$ defined as follows. Write $D=m d+r$, where $m \in \mathbb{N}$ and $r \in\{0, \ldots, d-1\}$. Then $E\left(\mathscr{C}_{D}(G)\right)$ is obtained from $E$ by duplicating each edge $m$ times and adding $r$ self loops to each vertex in $V$, i.e.,

$$
\forall(x, y) \in V \times V, \quad E\left(\mathscr{C}_{D}(G)\right)(x, y) \stackrel{\text { def }}{=} m E(x, y)+r \mathbf{1}_{\{x=y\}} .
$$

This definition makes $\mathscr{C}_{D}(G)$ be a $D$-regular graph.

Lemma 2.9. Fix two integers $D \geqslant d \geqslant 2$ and let $G=(V, E)$ be a d-regular graph. Then for every kernel $K: X \times X \rightarrow[0, \infty)$ we have

$$
\max \left\{\frac{\gamma\left(\mathscr{C}_{D}(G), K\right)}{\gamma(G, K)}, \frac{\gamma_{+}\left(\mathscr{C}_{D}(G), K\right)}{\gamma_{+}(G, K)}\right\} \leqslant 2 .
$$

Proof. Write $|V|=n$ and $D=m d+r$, where $m \in \mathbb{N}$ and $r \in\{0, \ldots, d-1\}$. For every $f, g: V \rightarrow X$ we have

$$
\begin{array}{r}
\frac{1}{n D} \sum_{(x, y) \in E\left(\mathscr{C}_{D}(G)\right)} K(f(x), g(y)) \stackrel{(46)}{=} \frac{1}{n d} \sum_{(x, y) \in V \times V} \frac{m d E(x, y)+r d \mathbf{1}_{\{x=y\}}}{m d+r} K(f(x), g(y)) \\
\geqslant \frac{1}{n d} \sum_{(x, y) \in V \times V} \frac{m}{m+1} E(x, y) K(f(x), g(y)) \geqslant \frac{1}{2} \cdot \frac{1}{n d} \sum_{(x, y) \in E} K(f(x), g(y)) .
\end{array}
$$




\section{Metric Markov Cotype implies nonlinear SPECTRAl CALCUlus}

Our goal here is to prove Theorem 1.7. We start with an analogous statement that treats the parameter $\gamma(\cdot, \cdot)$ rather than $\gamma_{+}(\cdot, \cdot)$.

Lemma 3.1 (Metric Markov cotype implies the decay of $\gamma$ ). Fix $C, \varepsilon \in(0, \infty), q \in[1, \infty)$, $m, n \in \mathbb{N}$ and an $n \times n$ symmetric stochastic matrix $A=\left(a_{i j}\right)$. Suppose that $\left(X, d_{X}\right)$ is a metric space such that for every $x_{1}, \ldots, x_{n} \in X$ there exist $y_{1}, \ldots, y_{n} \in X$ satisfying

$$
\sum_{i=1}^{n} d_{X}\left(x_{i}, y_{i}\right)^{q}+m^{\varepsilon} \sum_{i=1}^{n} \sum_{j=1}^{n} a_{i j} d_{X}\left(y_{i}, y_{j}\right)^{q} \leqslant C^{q} \sum_{i=1}^{n} \sum_{j=1}^{n} \mathscr{A}_{m}(A)_{i j} d_{X}\left(x_{i}, x_{j}\right)^{q} .
$$

Then

$$
\gamma\left(\mathscr{A}_{m}(A), d_{X}^{q}\right) \leqslant(3 C)^{q} \max \left\{1, \frac{\gamma\left(A, d_{X}^{q}\right)}{m^{\varepsilon}}\right\} .
$$

Proof. Write $B=\left(b_{i j}\right)=\mathscr{A}_{m}(A)$. If $\gamma\left(B, d_{X}^{q}\right) \leqslant(3 C)^{q}$ then (49) holds true, so we may assume from now on that $\gamma\left(B, d_{X}^{q}\right)>(3 C)^{q}$. Fix

$$
(3 C)^{q}<\gamma<\gamma\left(B, d_{X}^{q}\right) .
$$

By the definition of $\gamma\left(B, d_{X}^{q}\right)$ there exist $x_{1}, \ldots, x_{n} \in X$ such that

$$
\frac{1}{n^{2}} \sum_{i=1}^{n} \sum_{j=1}^{n} d_{X}\left(x_{i}, x_{j}\right)^{q}>\frac{\gamma}{n} \sum_{i=1}^{n} \sum_{j=1}^{n} b_{i j} d_{X}\left(x_{i}, x_{j}\right)^{q}
$$

Let $y_{1}, \ldots, y_{n} \in X$ satisfy (48). By the triangle inequality, for every $i, j \in\{1, \ldots, n\}$ we have

$$
d_{X}\left(x_{i}, x_{j}\right)^{q} \leqslant 3^{q-1}\left(d_{X}\left(x_{i}, y_{i}\right)^{q}+d_{X}\left(y_{i}, y_{j}\right)^{q}+d_{X}\left(y_{j}, x_{j}\right)^{q}\right) .
$$

By averaging (52) we get the following estimate.

$$
\begin{aligned}
\frac{1}{n^{2}} \sum_{i=1}^{n} \sum_{j=1}^{n} d_{X}\left(y_{i}, y_{j}\right)^{q} & \geqslant \frac{1}{3^{q-1} n^{2}} \sum_{i=1}^{n} \sum_{j=1}^{n} d_{X}\left(x_{i}, x_{j}\right)^{q}-\frac{2}{n} \sum_{i=1}^{n} d_{X}\left(x_{i}, y_{i}\right)^{q} \\
& \stackrel{(51)}{>} \frac{\gamma}{3^{q-1} n} \sum_{i=1}^{n} \sum_{j=1}^{n} b_{i j} d_{X}\left(x_{i}, x_{j}\right)^{q}-\frac{2}{n} \sum_{i=1}^{n} d_{X}\left(x_{i}, y_{i}\right)^{q} \\
& \geqslant \frac{3 \gamma m^{\varepsilon}}{(3 C)^{q} n} \sum_{i=1}^{n} \sum_{j=1}^{n} a_{i j} d_{X}\left(y_{i}, y_{j}\right)^{q}+\left(\frac{3 \gamma}{(3 C)^{q} n}-\frac{2}{n}\right) \sum_{i=1}^{n} d_{X}\left(x_{i}, y_{i}\right)^{q} \\
& \geqslant \frac{3 \gamma m^{\varepsilon}}{(3 C)^{q} n} \sum_{i=1}^{n} \sum_{j=1}^{n} a_{i j} d_{X}\left(y_{i}, y_{j}\right)^{q} .
\end{aligned}
$$

At the same time, by the definition of $\gamma\left(A, d_{X}^{q}\right)$ we have

$$
\frac{1}{n^{2}} \sum_{i=1}^{n} \sum_{j=1}^{n} d_{X}\left(y_{i}, y_{j}\right)^{q} \leqslant \frac{\gamma\left(A, d_{X}^{q}\right)}{n} \sum_{i=1}^{n} \sum_{j=1}^{n} a_{i j} d_{X}\left(y_{i}, y_{j}\right)^{q} .
$$

By contrasting (54) with (53) and letting $\gamma \nearrow \gamma\left(B, d_{X}^{p}\right)$ we deduce that

$$
\gamma\left(\mathscr{A}_{m}(A), d_{X}^{q}\right)=\gamma\left(B, d_{X}^{q}\right) \leqslant 3^{q-1} C^{q} \frac{\gamma\left(A, d_{X}^{q}\right)}{m^{\varepsilon}} .
$$


The special case $q=2$ of the following theorem implies Theorem 1.7.

Theorem 3.2 (Metric Markov cotype implies the decay of $\gamma_{+}$). Fix $C, \varepsilon \in(0, \infty), q \in[1, \infty)$, $m, n \in \mathbb{N}$ and an $n \times n$ symmetric stochastic matrix $A=\left(a_{i j}\right)$. Suppose that $\left(X, d_{X}\right)$ is a metric space such that for every $x_{1}, \ldots, x_{2 n} \in X$ there exist $y_{1}, \ldots, y_{2 n} \in X$ satisfying

$$
\sum_{i=1}^{2 n} d_{X}\left(x_{i}, y_{i}\right)^{q}+m^{\varepsilon} \sum_{i=1}^{2 n} \sum_{j=1}^{2 n}\left(\begin{array}{cc}
0 & A \\
A & 0
\end{array}\right)_{i j} d_{X}\left(y_{i}, y_{j}\right)^{q} \leqslant C^{q} \sum_{i=1}^{2 n} \sum_{j=1}^{2 n} \mathscr{A}_{m}\left(\begin{array}{ll}
0 & A \\
A & 0
\end{array}\right)_{i j} d_{X}\left(x_{i}, x_{j}\right)^{q} .
$$

Then

$$
\gamma_{+}\left(\mathscr{A}_{m}(A), d_{X}^{q}\right) \leqslant(45 C)^{q} \max \left\{1, \frac{\gamma_{+}\left(A, d_{X}^{q}\right)}{m^{\varepsilon}}\right\} .
$$

Proof. By Lemma 2.3 and Lemma 2.4 we have

$$
\gamma_{+}\left(\mathscr{A}_{m}(A), d_{X}^{q}\right) \stackrel{\sqrt{32})}{\leqslant} 2 \gamma\left(\left(\begin{array}{cc}
0 & \mathscr{A}_{m}(A) \\
\mathscr{A}_{m}(A) & 0
\end{array}\right), d_{X}^{q}\right) \stackrel{(35)}{\leqslant} 2\left(2^{q+1}+1\right) \gamma\left(\mathscr{A}_{m}\left(\begin{array}{cc}
0 & A \\
A & 0
\end{array}\right), d_{X}^{q}\right) .
$$

At the same time, an application of Lemma 3.1 and Lemma 2.3 yields the estimate

$$
\begin{aligned}
\gamma\left(\mathscr{A}_{m}\left(\begin{array}{ll}
0 & A \\
A & 0
\end{array}\right), d_{X}^{q}\right) \leqslant(3 C)^{q} \max \left\{1, \frac{\gamma\left(\left(\begin{array}{ll}
0 & A \\
A & 0
\end{array}\right), d_{X}^{q}\right)}{m^{\varepsilon}}\right\} \\
\stackrel{(321)}{\leqslant}(3 C)^{q} \max \left\{1, \frac{2^{q}+1}{2} \cdot \frac{\gamma_{+}\left(A, d_{X}^{q}\right)}{m^{\varepsilon}}\right\} .
\end{aligned}
$$

The desired estimate (56) is a consequence of (157) and (58).

\section{An iterative CONSTRUCtion OF SUPER-EXPANDERS}

Our goal here is to prove the existence of super-expanders as stated in Theorem 1.1, assuming the validity of Lemma 1.12, Corollary 1.10 and Theorem 1.13, These ingredients will then be proved in the subsequent sections.

In order to elucidate the ensuing construction, we phrase it in the setting of abstract kernels, though readers are encouraged to keep in mind that it will be used in the geometrically meaningful case of super-reflexive Banach spaces.

Lemma 4.1 (Initial zigzag iteration). Fix $d, m, t \in \mathbb{N}$ satisfying

$$
t d^{2(t-1)} \leqslant m
$$

and fix a d-regular graph $G_{0}=(V, E)$ with $|V|=m$. Then for every $j \in \mathbb{N}$ there exists a regular graph $F_{j}^{t}=\left(V_{j}^{t}, E_{j}^{t}\right)$ of degree $d^{2}$ and with $\left|V_{j}^{t}\right|=m^{j}$ such that the following holds true. If $K: X \times X \rightarrow[0, \infty)$ is a kernel such that $\gamma_{+}\left(G_{0}, K\right)<\infty$ then also $\gamma_{+}\left(F_{j}^{t}, K\right)<\infty$ for all $j \in \mathbb{N}$. Moreover, suppose that $C, \gamma \in[1, \infty)$ and $\varepsilon \in(0,1)$ satisfy

$$
t \geqslant\left(2 C \gamma^{2}\right)^{1 / \varepsilon}
$$

and that the kernel $K$ is such that every finite regular graph $G$ satisfies the nonlinear spectral calculus inequality

$$
\gamma_{+}\left(\mathscr{A}_{t}(G), K\right) \leqslant C \max \left\{1, \frac{\gamma_{+}(G, K)}{t^{\varepsilon}}\right\}
$$

Suppose furthermore that

$$
\gamma_{+}\left(G_{0}, K\right) \leqslant \gamma
$$


Then

$$
\sup _{j \in \mathbb{N}} \gamma_{+}\left(F_{j}^{t}, K\right) \leqslant 2 C \gamma^{2}
$$

Proof. Set $F_{1}^{t} \stackrel{\text { def }}{=} \mathscr{C}_{d^{2}}\left(G_{0}\right)$, where we recall the definition of the edge completion operation as discussed in Section 2.3. Thus $F_{1}^{t}$ has $m$ vertices and degree $d^{2}$. Assume inductively that we defined $F_{j}^{t}$ to be a regular graph with $m^{j}$ vertices and degree $d^{2}$. Then the Cesàro average $\mathscr{A}_{t}\left(F_{j}^{t}\right)$ has $m^{j}$ vertices and degree $t d^{2(t-1)}$ (recall the discussion preceding (16)). It follows from (59) that the degree of $\mathscr{A}_{t}\left(F_{j}^{t}\right)$ is at most $m$, so we can form the edge completion $\mathscr{C}_{m}\left(\mathscr{A}_{t}\left(F_{j}^{t}\right)\right)$, which has degree $m$, and we can therefore form the zigzag product

$$
F_{j+1}^{t} \stackrel{\text { def }}{=}\left(\mathscr{C}_{m}\left(\mathscr{A}_{t}\left(F_{j}^{t}\right)\right)\right) \text { (2) } G_{0} .
$$

Thus $F_{j+1}^{t}$ has $m^{j+1}$ vertices and degree $d^{2}$, completing the inductive construction. Using Theorem 1.3 and Lemma 2.9, it follows inductively that if $K: X \times X \rightarrow[0, \infty)$ is a kernel such that $\gamma_{+}\left(G_{0}, K\right)<\infty$ then also $\gamma_{+}\left(F_{j}^{t}, K\right)<\infty$ for all $j \in \mathbb{N}$.

Assuming the validity of (62), by Lemma 2.9 we have

$$
\gamma_{+}\left(F_{1}^{t}, K\right)=\gamma_{+}\left(\mathscr{C}_{d^{2}}\left(G_{0}\right), K\right) \stackrel{(477)}{\leqslant} 2 \gamma_{+}\left(G_{0}, K\right) \stackrel{\sqrt[626]{62}}{\leqslant} 2 \gamma .
$$

We claim that for every $j \in \mathbb{N}$,

$$
\gamma_{+}\left(F_{j}^{t}, K\right) \leqslant 2 C \gamma^{2} .
$$

Assuming the validity of (64) for some $j \in \mathbb{N}$, by Theorem 1.3 we have

$$
\begin{aligned}
& \gamma_{+}\left(F_{j+1}^{t}, K\right) \stackrel{(12) \wedge[63]}{\leqslant} \gamma_{+}\left(\mathscr{C}_{m}\left(\mathscr{A}_{t}\left(F_{j}^{t}\right)\right)\right) \gamma_{+}\left(G_{0}, K\right)^{2} \stackrel{(477) \wedge \sqrt{620}}{\leqslant} 2 \gamma_{+}\left(\mathscr{A}_{t}\left(F_{j}^{t}\right), K\right) \gamma^{2} \\
& \stackrel{\sqrt[6110]{ }}{\leqslant} 2 C \gamma^{2} \max \left\{1, \frac{\gamma_{+}\left(F_{j}^{t}, K\right)}{t^{\varepsilon}}\right\} \stackrel{\sqrt[644]{\leqslant}}{\leqslant} 2 C \gamma^{2} \max \left\{1, \frac{2 C \gamma^{2}}{t^{\varepsilon}}\right\} \stackrel{\sqrt[600]{\leqslant}}{\leqslant} 2 C \gamma^{2} \text {. }
\end{aligned}
$$

Corollary 4.2 (Intermediate construction for super-reflexive Banach spaces). For every $k \in \mathbb{N}$ there exist regular graphs $\left\{F_{j}(k)\right\}_{j=1}^{\infty}$ and integers $\left\{d_{k}\right\}_{k=1}^{\infty},\left\{n_{j}(k)\right\}_{j, k \in \mathbb{N}} \subseteq \mathbb{N}$, where $\left\{n_{j}(k)\right\}_{j=1}^{\infty}$ is a strictly increasing sequence, such that $F_{j}(k)$ has degree $d_{k}$ and $n_{j}(k)$ vertices, and the following condition holds true. For every super-reflexive Banach space $\left(X,\|\cdot\|_{X}\right)$,

$$
\forall j, k \in \mathbb{N}, \quad \gamma_{+}\left(F_{j}(k),\|\cdot\|_{X}^{2}\right)<\infty,
$$

and moreover there exists $k(X) \in \mathbb{N}$ such that

$$
\sup _{\substack{j, k \in \mathbb{N} \\ k \geqslant k(X)}} \gamma_{+}\left(F_{j}(k),\|\cdot\|_{X}^{2}\right) \leqslant k(X) .
$$

Proof. We shall use here the notation of Lemma 1.12. For every $k \in \mathbb{N}$ choose an integer $n(k) \geqslant n_{0}(1 / k)$ (recall that $n_{0}(1 / k)$ was introduced in Lemma 1.12) such that

$$
k e^{2(k-1)\left(\log m_{n(k)}\right)^{1-\frac{1}{k}}} \leqslant m_{n(k)} .
$$

By (19)), it follows from (65) that $d_{n(k)}(1 / k)$, i.e., the degree of the graph $H_{n(k)}(1 / k)$, satisfies

$$
k d_{n(k)}^{2(k-1)} \leqslant m_{n(k)}=\left|V\left(H_{n(k)}(1 / k)\right)\right|,
$$


where here, and in what follows, $V(G)$ denotes the set of vertices of a graph $G$. We can therefore apply Lemma 4.1 with the parameters $t=k, d=d_{n(k)}(1 / k), m=m_{n(k)}$ and $G_{0}=H_{n(k)}(1 / k)$. Letting $\left\{F_{j}(k)\right\}_{j=1}^{\infty}$ denote the resulting sequence of graphs, we define

$$
d_{k} \stackrel{\text { def }}{=}\left(d_{n(k)}(1 / k)\right)^{2} \quad \text { and } \quad n_{j}(k) \stackrel{\text { def }}{=}\left(m_{n(k)}\right)^{j} \text {. }
$$

If $\left(X,\|\cdot\|_{X}\right)$ is a super-reflexive Banach space then it is in particular $K$-convex (see [58]). Recalling the parameter $\delta_{0}(X)$ of Lemma 1.12, we have

$$
k \geqslant \frac{1}{\delta_{0}(X)} \Longrightarrow \gamma_{+}\left(H_{n(k)}(1 / k),\|\cdot\|_{X}^{2}\right) \leqslant 9^{3} .
$$

It also follows from Corollary 1.10 that there exists $C(X) \in[1, \infty)$ and $\varepsilon(X) \in(0,1)$ for which every finite regular graph $G$ satisfies

$$
\forall t \in \mathbb{N}, \quad \gamma_{+}\left(\mathscr{A}_{t}(G),\|\cdot\|_{X}^{2}\right) \leqslant C(X) \max \left\{1, \frac{\gamma_{+}\left(G,\|\cdot\|_{X}^{2}\right)}{t^{\varepsilon(X)}}\right\} .
$$

We may therefore apply Lemma 4.1 with $C=C(X), \varepsilon=\varepsilon(X)$ and $\gamma=9^{3}$ to deduce that if we define

$$
k(X) \stackrel{\text { def }}{=}\left\lceil\max \left\{\frac{1}{\delta_{0}(X)},\left(2 C(X) \cdot 9^{3}\right)^{1 / \varepsilon(X)}, 2 C(X) \cdot 9^{6}\right\}\right\rceil,
$$

then for every $j \in \mathbb{N}$,

$$
k \geqslant k(X) \Longrightarrow \sup _{j \in \mathbb{N}} \gamma_{+}\left(F_{j}(k),\|\cdot\|_{X}^{2}\right) \leqslant 2 C(X) \cdot 9^{6} \leqslant k(X) .
$$

Corollary 4.2 provides a sequence of expanders with respect to a fixed super-reflexive Banach space $\left(X,\|\cdot\|_{X}\right)$, but since the sequence of degrees $\left\{d_{k}\right\}_{k=1}^{\infty}$ may be unbounded (this is indeed the case in our construction), we still do not have one sequence of bounded degree regular graphs that are expanders with respect to every super-reflexive Banach space. This is achieved in the following crucial lemma.

Lemma 4.3 (Main zigzag iteration). Let $\left\{d_{k}\right\}_{k=1}^{\infty}$ be a sequence of integers and for each $k \in \mathbb{N}$ let $\left\{n_{j}(k)\right\}_{j=1}^{\infty}$ be a strictly increasing sequence of integers. For every $j, k \in \mathbb{N}$ let $F_{j}(k)$ be a regular graph of degree $d_{k}$ with $n_{j}(k)$ vertices. Suppose that $\mathscr{K}$ is a family of kernels such that

$$
\forall K \in \mathscr{K}, \forall j, k \in \mathbb{N}, \quad \gamma_{+}\left(F_{j}(k), K\right)<\infty .
$$

Suppose also that the following two conditions hold true.

- For every $K \in \mathscr{K}$ there exists $k_{1}(K) \in \mathbb{N}$ such that

$$
\sup _{\substack{j, k \in \mathbb{N} \\ k \geqslant k_{1}(K)}} \gamma_{+}\left(F_{j}(k), K\right) \leqslant k_{1}(K) .
$$

- For every $K \in \mathscr{K}$ there exists $k_{2}(K) \in \mathbb{N}$ such that every regular graph $G$ satisfies the following spectral calculus inequality.

$$
\forall t \in \mathbb{N}, \quad \gamma_{+}\left(\mathscr{A}_{t}(G), K\right) \leqslant k_{2}(K) \max \left\{1, \frac{\gamma_{+}(G, K)}{t^{1 / k_{2}(K)}}\right\} .
$$


Then there exists $d \in \mathbb{N}$ and a sequence of $d$-regular graphs $\left\{H_{i}\right\}_{i=1}^{\infty}$ with

$$
\lim _{i \rightarrow \infty}\left|V\left(H_{i}\right)\right|=\infty
$$

and

$$
\forall K \in \mathscr{K}, \quad \sup _{j \in \mathbb{N}} \gamma_{+}\left(H_{j}, K\right)<\infty .
$$

Proof. In what follows, for every $k \in \mathbb{N}$ it will be convenient to introduce the notation

$$
M_{k} \stackrel{\text { def }}{=}\left(2 k^{3}\right)^{k} \text {. }
$$

With this, define

$$
j(k) \stackrel{\text { def }}{=} \min \left\{j \in \mathbb{N}: n_{j}(k)>2 d_{1}^{2}+M_{k+1} d_{k+1}^{2\left(M_{k+1}-1\right)}\right\},
$$

and

$$
W_{k} \stackrel{\text { def }}{=} F_{j(k)}(k) \text {. }
$$

We will next define for every $k \in \mathbb{N}$ an integer $\ell(k) \in \mathbb{N} \cup\{0\}$ and a sequence of regular graphs $W_{k}^{0}, W_{k}^{1}, \ldots, W_{k}^{\ell(k)}$, along with an auxiliary integer sequence $\left\{h_{i}(k)\right\}_{i=0}^{\ell(k)} \subseteq \mathbb{N}$. Set

$$
W_{k}^{0} \stackrel{\text { def }}{=} W_{k} \quad \text { and } \quad h_{0}(k) \stackrel{\text { def }}{=} k .
$$

Define $\ell(1)=0$. For every integer $k>1$ set

$$
h_{1}(k) \stackrel{\text { def }}{=} \min \left\{h \in \mathbb{N}: n_{j(h)}(h) \geqslant d_{h_{0}(k)}\right\} .
$$

Observe that necessarily $h_{1}(k)<h_{0}(k)=k$. Indeed, if $h_{1}(k) \geqslant k$ then

$$
d_{k} \stackrel{(75)}{>} n_{j(k-1)}(k-1) \stackrel{(722)}{>} M_{k} d_{k}^{2\left(M_{k}-1\right)} \stackrel{(71)}{\geqslant} d_{k}
$$

a contradiction. By the definition of $h_{1}(k)$ we know that $n_{j\left(h_{1}(k)\right)}\left(h_{1}(k)\right) \geqslant d_{h_{0}(k)}$, so we may form the edge completion $\mathscr{C}_{n_{j\left(h_{1}(k)\right)}\left(h_{1}(k)\right)}\left(W_{k}^{0}\right)$. Since the number of vertices of $W_{h_{1}(k)}$ is $n_{j\left(h_{1}(k)\right)}\left(h_{1}(k)\right)$, which is the same as the degree of $\mathscr{C}_{n_{j\left(h_{1}(k)\right)}\left(h_{1}(k)\right)}\left(W_{k}^{0}\right)$, we can define

$$
W_{k}^{1} \stackrel{\text { def }}{=} \mathscr{A}_{M_{h_{1}(k)}}\left(\mathscr{C}_{n_{j\left(h_{1}(k)\right)}\left(h_{1}(k)\right)}\left(W_{k}^{0}\right)(2) W_{h_{1}(k)}\right) \text {. }
$$

The degree of $W_{k}^{1}$ equals

$$
M_{h_{1}(k)} d_{h_{1}(k)}^{2\left(M_{h_{1}(k)}-1\right)} .
$$

Assume inductively that $k, i>1$ and we have already defined the graph $W_{k}^{i-1}$ and the integer $h_{i-1}(k)$, such that the degree of $W_{k}^{i-1}$ equals

$$
M_{h_{i-1}(k)} d_{h_{i-1}(k)}^{2\left(M_{h_{i-1}(k)}-1\right)} .
$$

If $h_{i-1}(k)=1$ then conclude the construction, setting $\ell(k)=i-1$. If $h_{i-1}(k)>1$ then we proceed by defining

$$
h_{i}(k) \stackrel{\text { def }}{=} \min \left\{h \in \mathbb{N}: n_{j(h)}(h) \geqslant M_{h_{i-1}(k)} d_{h_{i-1}(k)}^{2\left(M_{h_{i-1}(k)}-1\right)}\right\} .
$$

Observe that

$$
h_{i}(k)<h_{i-1}(k)
$$


Indeed, if $h_{i}(k) \geqslant h_{i-1}(k)$ then

$$
M_{h_{i-1}(k)} d_{h_{i-1}(k)}^{2\left(M_{h_{i-1}(k)}-1\right)} \stackrel{(77)}{>} n_{j\left(h_{i-1}(k)-1\right)}\left(h_{i-1}(k)-1\right) \stackrel{\sqrt{72})}{>} 2 d_{1}^{2}+M_{h_{i-1}(k)} d_{h_{i-1}(k)}^{2\left(M_{h_{i-1}(k)}-1\right)},
$$

a contradiction. Since the degree of $W_{k}^{i-1}$ is given in (76), which by (77) is at most $n_{j\left(h_{i}(k)\right)}\left(h_{i}(k)\right)$, we may form the edge completion $\mathscr{C}_{n_{j\left(h_{i}(k)\right)}\left(h_{i}(k)\right)}\left(W_{k}^{i-1}\right)$. The degree of the resulting graph is $n_{j\left(h_{i}(k)\right)}\left(h_{i}(k)\right)$, which, by (73), equals the number of vertices of $W_{h_{i}(k)}$. We can therefore define

$$
W_{k}^{i} \stackrel{\text { def }}{=} \mathscr{A}_{M_{h_{i}(k)}}\left(\mathscr{C}_{n_{j\left(h_{i}(k)\right)}\left(h_{i}(k)\right)}\left(W_{k}^{i-1}\right)(2) W_{h_{i}(k)}\right) .
$$

The degree of $W_{k}^{i}$ equals $M_{h_{i}(k)} d_{h_{i}(k)}^{2\left(M_{h_{i}(k)}-1\right)}$, thus completing the inductive step.

Due to (78) the above procedure must eventually terminate, and by definition $h_{\ell(k)}(k)=1$. Since $h_{0}(k)=k$, it follows that

$$
\forall k \in \mathbb{N}, \quad \ell(k) \leqslant k .
$$

We define

$$
H_{k} \stackrel{\text { def }}{=} W_{k}^{\ell(k)} .
$$

The degree of $H_{k}$ equals $d \stackrel{\text { def }}{=} 2 d_{1}^{2}$ for all $k \in \mathbb{N}$. Also, by construction we have

$$
\left|V\left(H_{k}\right)\right|=\left|V\left(W_{k}^{\ell(k)}\right)\right| \geqslant\left|V\left(W_{k}^{\ell(k)-1}\right)\right| \geqslant \ldots \geqslant\left|V\left(W_{k}^{0}\right)\right| \stackrel{(74) \wedge(\sqrt{73})}{=} n_{j(k)}(k) \stackrel{\text { (72) }}{\geqslant} M_{k+1} .
$$

Thus $\lim _{k \rightarrow \infty}\left|V\left(H_{k}\right)\right|=\infty$. It remains to prove that for every kernel $K \in \mathscr{K}$ we have

$$
\sup _{k \in \mathbb{N}} \gamma_{+}\left(H_{k}, K\right)<\infty .
$$

To prove (81) we start with the following crucial estimate, which holds for every $k \in \mathbb{N}$ and $i \in\{1, \ldots, \ell(k)\}$.

$$
\begin{aligned}
& \gamma_{+}\left(W_{k}^{i}, K\right) \stackrel{(69) \wedge(79)}{\leqslant} k_{2}(K) \max \left\{1, \frac{\gamma_{+}\left(\mathscr{C}_{n_{j\left(h_{i}(k)\right)}\left(h_{i}(k)\right)}\left(W_{k}^{i-1}\right)\left(\mathrm{Z} W_{h_{i}(k)}, K\right)\right.}{M_{h_{i}(k)}^{1 / k_{2}(K)}}\right\} \\
& \stackrel{\text { (12) }}{\wedge(477) \wedge(73)} k_{2}(K) \max \left\{1, \frac{2 \gamma_{+}\left(W_{k}^{i-1}, K\right) \gamma_{+}\left(F_{j\left(h_{i}(k)\right)}\left(h_{i}(k)\right), K\right)^{2}}{M_{h_{i}(k)}^{1 / k_{2}(K)}}\right\} \text {. }
\end{aligned}
$$

In particular, it follows from (82) that the following crude estimate holds true.

$$
\gamma_{+}\left(W_{k}^{i}, K\right) \leqslant 2 k_{2}(K) \gamma_{+}\left(W_{k}^{i-1}, K\right) \gamma_{+}\left(F_{j\left(h_{i}(k)\right)}\left(h_{i}(k)\right), K\right)^{2} .
$$

A recursive application of (83) yields the estimate

$$
\gamma_{+}\left(H_{k}, K\right)=\gamma_{+}\left(W_{k}^{\ell(k)}, K\right) \leqslant\left(2 k_{2}(K)\right)^{\ell(k)} \gamma_{+}\left(W_{k}^{0}, K\right) \prod_{i=1}^{\ell(k)} \gamma_{+}\left(F_{j\left(h_{i}(k)\right)}\left(h_{i}(k)\right), K\right)^{2} .
$$

Due to the finiteness assumption (67), it follows that

$$
\forall k \in \mathbb{N}, \quad \gamma_{+}\left(H_{k}, K\right)<\infty .
$$

In order to prove (81) we will need to apply (82) more carefully. To this end set

$$
k_{3}(K) \stackrel{\text { def }}{=} \max \left\{k_{1}(K), k_{2}(K)\right\} \text {, }
$$


and fix $k>k_{3}(K)$. We will now prove by induction on $i \in\{0, \ldots, \ell(k)\}$ that

$$
h_{i}(k)>k_{3}(K) \Longrightarrow \gamma_{+}\left(W_{k}^{i}, K\right) \leqslant k_{3}(K) .
$$

If $i=0$ then $h_{0}(k)=k>k_{3}(K) \geqslant k_{1}(K)$, so by our assumption (68),

$$
\gamma_{+}\left(W_{k}^{0}, K\right) \stackrel{[(74)}{=} \stackrel{(73)}{=} \gamma_{+}\left(F_{j(k)}(k), K\right) \stackrel{(68)}{\leqslant} k_{1}(K) \leqslant k_{3}(K) .
$$

Assume inductively that $i \in\{1, \ldots, \ell(k)\}$ satisfies

$$
h_{i}(k)>k_{3}(K) \text {. }
$$

By (78) and the inductive hypothesis we therefore have

$$
\gamma_{+}\left(W_{k}^{i-1}, K\right) \leqslant k_{3}(K)
$$

Hence,

$$
\begin{aligned}
& \gamma_{+}\left(W_{k}^{i}, K\right) \stackrel{(82) \wedge(87) \wedge(\sqrt{(68) \wedge} \leqslant}{\leqslant} k_{2}(K) \max \left\{1, \frac{2 k_{3}(K) k_{1}(K)^{2}}{M_{h_{i}(k)}^{1 / k_{2}(K)}}\right\} \\
& \stackrel{[85)}{\wedge} k_{3}(K) \max \left\{1, \frac{2 k_{3}(K)^{3}}{M_{k_{3}(K)}^{1 / k_{3}(K)}}\right\} \stackrel{(71)}{=} k_{3}(K) .
\end{aligned}
$$

This completes the inductive proof of (86 $)$.

Define

$$
i_{0}(k) \stackrel{\text { def }}{=} \max \left\{i \in\{0, \ldots, \ell(k)-1\}: h_{i}(k)>k_{3}(K)\right\} .
$$

Note that since $h_{0}(k)=k$, the maximum in (89) is well defined. By (86) we have

$$
\gamma_{+}\left(W_{k}^{i_{0}(k)}, K\right) \leqslant k_{3}(K)
$$

A recursive application of (83), combined with (90), yields the estimate

$$
\gamma_{+}\left(H_{k}, K\right) \leqslant k_{3}(K) \prod_{i=i_{0}(k)+1}^{\ell(k)}\left(2 k_{2}(K) \gamma_{+}\left(F_{j\left(h_{i}(k)\right)}\left(h_{i}(k)\right), K\right)^{2}\right) .
$$

By (89), for every $i \in\left\{i_{0}(k)+1, \ldots, \ell(k)\right\}$ we have $h_{i}(k) \leqslant k_{3}(K)$. Due to the strict monotonicity appearing in (78), it follows that the number of terms in the product appearing in (91) is at most $k_{3}(K)$, and therefore

$$
\gamma_{+}\left(H_{k}, K\right) \leqslant k_{3}(K)\left(2 k_{2}(K)\right)^{k_{3}(K)} \prod_{r=1}^{k_{3}(K)} \gamma_{+}\left(F_{j(r)}(r), K\right)^{2} .
$$

We have proved that (92) holds true for every integer $k>k_{3}(K)$. Note that the upper bound in (92) is independent of $k$, so in combination with (84) this completes the proof of (81).

Proof of Theorem 1.1. Lemma 4.3 applies when $\mathscr{K}$ consists of all $K: X \times X \rightarrow[0, \infty)$ of the form $K(x, y)=\|x-y\|_{X}^{2}$, where $\left(X,\|\cdot\|_{X}\right)$ ranges over all super-reflexive Banach spaces. Indeed, hypotheses (67) and (68) of Lemma 4.3 are nothing more than the assertions of 
Corollary 4.2. Hypothesis (69) of Lemma 4.3 holds true as well since, by Corollary 1.10, every super-reflexive Banach space $\left(X,\|\cdot\|_{X}\right)$ satisfies (66), so we may take

$$
k_{2}(X) \stackrel{\text { def }}{=} \max \left\{C(X), \frac{1}{\varepsilon(X)}\right\} .
$$

Let $d \in \mathbb{N}$ and $\left\{H_{i}\right\}_{i=1}^{\infty}$ be the output of Lemma4.3, Recalling the notation of Remark 2.2, $C_{d}^{\circ}$ denotes the cycle of length $d$ with self loops, and $C_{9}$ denotes the cycle of length 9 without self loops. For each $i \in \mathbb{N}$, since $H_{i}$ is $d$-regular, we may form the zigzag product $H_{i}$ (2) $C_{d}^{\circ}$, which is a 9-regular graph with $d\left|V\left(H_{i}\right)\right|$ vertices. We can therefore consider the graph

$$
H_{i}^{*} \stackrel{\text { def }}{=}\left(H_{i}(\mathrm{Z}) C_{d}^{\circ}\right) \text { ○ } C_{9} \text {. }
$$

Thus $\left\{H_{i}^{*}\right\}_{i=1}^{\infty}$ are 3-regular graphs with $\lim _{i \rightarrow \infty}\left|V\left(H_{i}^{*}\right)\right|=\infty$. By Theorem 1.3 and part (IV) of Theorem 1.13, for every super-reflexive Banach space $\left(X,\|\cdot\|_{X}\right)$ we have

$$
\gamma_{+}\left(H_{i}^{*},\|\cdot\|_{X}^{2}\right) \leqslant 9 \gamma_{+}\left(H_{i},\|\cdot\|_{X}^{2}\right) \gamma_{+}\left(C_{d}^{\circ},\|\cdot\|_{X}^{2}\right)^{2} \gamma_{+}\left(C_{9},\|\cdot\|_{X}^{2}\right)^{2} .
$$

By Lemma 2.1 we have $\gamma_{+}\left(C_{d}^{\circ},\|\cdot\|_{X}^{2}\right) \leqslant 12 d^{2}$ and $\gamma_{+}\left(C_{9},\|\cdot\|_{X}^{2}\right) \leqslant 648$ (since $C_{9}$ is not bipartite). Therefore $\gamma_{+}\left(H_{i}^{*},\|\cdot\|_{X}^{2}\right) \lesssim d^{4} \gamma_{+}\left(H_{i},\|\cdot\|_{X}^{2}\right)$, so due to (170) the graphs $\left\{H_{i}^{*}\right\}_{i=1}^{\infty}$ satisfy the conclusion of Theorem 1.1.

Remark 4.4. V. Lafforgue asked [28] whether there exists a sequence of bounded degree graphs $\left\{G_{k}\right\}_{k=1}^{\infty}$ that does not admit a coarse embedding (with the same moduli) into any $K$-convex Banach space. A positive answer to this question follows from our methods. Independently of our work, Lafforgue [29] managed to solve this problem as well, so we only sketch the argument. An inspection of Lafforgue's proof in [28] shows that his method produces regular graphs $\left\{H_{j}(k)\right\}_{j, k \in \mathbb{N}}$ such that for each $k \in \mathbb{N}$ the graphs $\left\{H_{j}(k)\right\}_{j \in \mathbb{N}}$ have degree $d_{k}$, their cardinalities are unbounded, and for every $K$-convex Banach space $\left(X,\|\cdot\|_{X}\right)$ there is some $k \in \mathbb{N}$ for which $\sup _{j \in \mathbb{N}} \gamma_{+}\left(H_{j}(k),\|\cdot\|_{X}^{2}\right)<\infty$. The problem is that the degrees $\left\{d_{k}\right\}_{k \in \mathbb{N}}$ are unbounded, but this can be overcome as above by applying the zigzag product with a cycle with self loops. Indeed, define $G_{j}(k)=H_{j}(k)$ (2) $C_{d_{k}}^{\circ}$. Then $G_{j}(k)$ is 9-regular, and as argued in the proof of Theorem 1.1, we still have $\sup _{j \in \mathbb{N}} \gamma_{+}\left(G_{j}(k),\|\cdot\|_{X}^{2}\right)<\infty$. To get a single sequence of graphs that does not admit a coarse embedding into any $K$-convex Banach space, fix a bijection $\psi=(a, b): \mathbb{N} \rightarrow \mathbb{N} \times \mathbb{N}$, and define $G_{m}=G_{a(m)}(b(m))$. The graphs $G_{m}$ all have degree 9 . If $X$ is $K$-convex then choose $k \in \mathbb{N}$ as above. If we let $m_{j} \in \mathbb{N}$ be such that $\psi\left(m_{j}\right)=(j, k)$ then we have shown that the graphs $\left\{G_{m_{j}}\right\}_{j=1}^{\infty}$ are arbitrarily large, have bounded degree, and satisfy $\sup _{j \in \mathbb{N}} \gamma_{+}\left(G_{m_{j}},\|\cdot\|_{X}^{2}\right)<\infty$. The argument that was presented in Section 1.1 implies that $\left\{G_{m}\right\}_{m=1}^{\infty}$ do not embed coarsely into $X$.

\section{The HeAT SEMigRoup ON THE TAIL SPACE}

This section contains estimates that will be crucially used in the proof of Lemma 1.12, in addition to geometric results and open questions of independent interest. We start the discussion by recalling some basic definitions, and setting some (mostly standard) notation on vector-valued Fourier analysis. Let $\left(X,\|\cdot\|_{X}\right)$ be a Banach space. We assume throughout that $X$ is a Banach space over the complex scalars, though, by a standard complexification argument, our results hold also for Banach spaces over $\mathbb{R}$. 
Given a measure space $(\Omega, \mu)$ and $p \in[1, \infty)$, we denote as usual by $L_{p}(\mu, X)$ the space of all measurable $f: \Omega \rightarrow X$ satisfying

$$
\|f\|_{L_{p}(\mu, X)} \stackrel{\text { def }}{=}\left(\int_{\Omega}\|f\|_{X}^{p} d \mu\right)^{1 / p}<\infty .
$$

When $X=\mathbb{C}$ we use the standard notation $L_{p}(\mu)=L_{p}(\mu, \mathbb{C})$. When $\Omega$ is a finite set we denote by $L_{p}(\Omega, X)$ the space $L_{p}(\mu, X)$, where $\mu$ is the normalized counting measure on $\Omega$.

For $n \in \mathbb{N}$ and $A \subseteq\{1, \ldots, n\}$, the Walsh function $W_{A}: \mathbb{F}_{2}^{n} \rightarrow\{-1,1\}$ is defined by

$$
W_{A}(x) \stackrel{\text { def }}{=}(-1)^{\sum_{j \in A} x_{j}} .
$$

Any $f: \mathbb{F}_{2}^{n} \rightarrow X$ has the expansion

$$
f=\sum_{A \subseteq\{1, \ldots, n\}} \widehat{f}(A) W_{A},
$$

where

$$
\widehat{f}(A) \stackrel{\text { def }}{=} \frac{1}{2^{n}} \sum_{x \in \mathbb{F}_{2}^{n}} f(x) W_{A}(x) \in X .
$$

For $\varphi: \mathbb{F}_{2}^{n} \rightarrow \mathbb{C}$ and $f: \mathbb{F}_{2}^{n} \rightarrow X$, the convolution $\varphi * f: \mathbb{F}_{2}^{n} \rightarrow X$ is defined as usual by

$$
\varphi * f(x) \stackrel{\text { def }}{=} \frac{1}{2^{n}} \sum_{w \in \mathbb{F}_{2}^{n}} \varphi(x-w) f(w)=\sum_{A \subseteq\{1, \ldots, n\}} \widehat{\varphi}(A) \widehat{f}(A) W_{A}(x) .
$$

For $k \in\{1, \ldots, n\}$ and $p \in[1, \infty]$ we let $L_{p}^{\geqslant k}\left(\mathbb{F}_{2}^{n}, X\right)$ denote the subspace of $L_{p}\left(\mathbb{F}_{2}^{n}, X\right)$ consisting of those $f: \mathbb{F}_{2}^{n} \rightarrow X$ that satisfy $\widehat{f}(A)=0$ for all $A \subseteq\{1, \ldots, n\}$ with $|A|<k$.

Let $e_{1}, \ldots, e_{n}$ be the standard basis of $\mathbb{F}_{2}^{n}$. For $j \in\{1, \ldots, n\}$ define $\partial_{j} f: \mathbb{F}_{2}^{n} \rightarrow X$ by

$$
\partial_{j} f(x) \stackrel{\text { def }}{=} \frac{f(x)-f\left(x+e_{j}\right)}{2} .
$$

Thus

$$
\partial_{j} f=\sum_{\substack{A \subseteq\{1, \ldots, n\} \\ j \in A}} \widehat{f}(A) W_{A}
$$

and

$$
\Delta f \stackrel{\text { def }}{=} \sum_{j=1}^{n} \partial_{j} f=\sum_{A \subseteq\{1, \ldots, n\}}|A| \widehat{f}(A) W_{A} .
$$

For every $z \in \mathbb{C}$ we then have

$$
e^{z \Delta} f=\sum_{A \subseteq\{1, \ldots, n\}} e^{z|A|} \widehat{f}(A) W_{A}=R_{z} * f,
$$

where

$$
R_{z}(x) \stackrel{\text { def }}{=} \prod_{j=1}^{n}\left(1+e^{z}(-1)^{x_{j}}\right)=\left(1-e^{z}\right)^{\|x\|_{1}}\left(1+e^{z}\right)^{n-\|x\|_{1}},
$$

and we identify $\mathbb{F}_{2}^{n}$ with $\{0,1\}^{n} \subseteq \mathbb{R}^{n}$. Hence, for every $x \in \mathbb{F}_{2}^{n}$ we have

$$
e^{z \Delta} f(x)=\sum_{w \in \mathbb{F}_{2}^{n}}\left(\frac{1-e^{z}}{2}\right)^{\|x-w\|_{1}}\left(\frac{1+e^{z}}{2}\right)^{n-\|x-w\|_{1}} f(w) .
$$


In particular,

$$
\forall x, y \in \mathbb{F}_{2}^{n}, \quad\left(e^{z \Delta} \delta_{x}\right)(y)=\left(\frac{1-e^{z}}{2}\right)^{\|x-y\|_{1}}\left(\frac{1+e^{z}}{2}\right)^{n-\|x-y\|_{1}},
$$

where $\delta_{x}(w) \stackrel{\text { def }}{=} \mathbf{1}_{\{x=w\}}$ is the Kronecker delta.

Given $n \in \mathbb{N}$ and $f: \mathbb{F}_{2}^{n} \rightarrow X$, the Rademacher projection [41] of $f$ is defined by

$$
\boldsymbol{\operatorname { R a d }}(f) \stackrel{\text { def }}{=} \sum_{j=1}^{n} \widehat{f}(\{j\}) W_{\{j\}} .
$$

The $K$-convexity constant of $X$ is defined [41] by

$$
K(X) \stackrel{\text { def }}{=} \sup _{n \in \mathbb{N}}\|\mathbf{R a d}\|_{L_{2}\left(\mathbb{F}_{2}^{n}, X\right) \rightarrow L_{2}\left(\mathbb{F}_{2}^{n}, X\right)} .
$$

If $K(X)<\infty$ then $X$ is said to be $K$-convex. Pisier's deep $K$-convexity theorem [58] asserts that $X$ is $K$-convex if and only if it does not contain copies of $\left\{\ell_{1}^{n}\right\}_{n=1}^{\infty}$ with distortion arbitrarily close to 1 , i.e., for all $n \in \mathbb{N}$ we have

$$
\inf _{T \in \mathscr{L}\left(\ell_{1}^{n}, X\right)}\|T\|_{\ell_{1}^{n} \rightarrow X} \cdot\left\|T^{-1}\right\|_{T\left(\ell_{1}^{n}\right) \rightarrow \ell_{1}^{n}}=1,
$$

where $\mathscr{L}\left(\ell_{1}^{n}, X\right)$ denotes the space of linear operators $T: \ell_{1}^{n} \rightarrow X$ (and we use the convention $\left\|T^{-1}\right\|_{T\left(\ell_{1}^{n}\right) \rightarrow \ell_{1}^{n}}=\infty$ if $T$ is not injective).

Our main result in this section is the following theorem.

Theorem 5.1 (Decay of the heat semigroup on the tail space). For every $K, p \in(1, \infty)$ there are $A(K, p) \in(0,1)$ and $B(K, p), C(K, p) \in(2, \infty)$ such that for every $K$-convex $\operatorname{Banach}\left(X,\|\cdot\|_{X}\right)$ with $K(X) \leqslant K$, every $k, n \in \mathbb{N}$ and every $t \in(0, \infty)$,

$$
\left\|e^{-t \Delta}\right\|_{L_{p}^{\geqslant k}\left(\mathbb{F}_{2}^{n}, X\right) \rightarrow L_{p}^{\geqslant k}\left(\mathbb{F}_{2}^{n}, X\right)} \leqslant C(K, p) e^{-A(K, p) k \min \left\{t, t^{B(K, p)}\right\}} .
$$

The fact that Theorem 5.1 assumes that $X$ is $K$-convex is not an artifact of our proof: we have, in fact, the following converse statement.

Theorem 5.2. Let $X$ be a Banach space $\left(X,\|\cdot\|_{X}\right)$ for which exist $k \in \mathbb{N}, p \in(1, \infty)$ and $t \in(0, \infty)$ such that

$$
\sup _{n \in \mathbb{N}}\left\|e^{-t \Delta}\right\|_{L_{p}^{\geqslant k}\left(\mathbb{F}_{2}^{n}, X\right) \rightarrow L_{p}^{\geqslant k}\left(\mathbb{F}_{2}^{n}, X\right)}<1 .
$$

Then $X$ is $K$-convex.

Remark 5.3. We conjecture that any $K$-convex Banach space satisfies (98) for every $k \in \mathbb{N}$, $p \in(1, \infty)$ and $t \in(0, \infty)$. Theorem 5.1 implies (98) if $k$ or $t$ are large enough, but, due to the factor $C(K, p)$ in (97), it does not imply (98) in its entirety. The factor $C(K, p)$ in (97) does not have impact on the application of Theorem [5.1 that we present here; see Section 7.

5.1. Warmup: the tail space of scalar valued functions. Before passing to the proofs of Theorem 5.1 and Theorem 5.2, we address separately the classical scalar case $X=\mathbb{C}$, since it already exhibits interesting open questions. The problem was studied by P.-A. Meyer [46] who proved Lemma 5.4 below. We include its proof here since it is not stated explicitly in this way in [46, and moreover Meyer studies this problem with $\mathbb{F}_{2}^{n}$ replaced by $\mathbb{R}^{n}$ equipped with the standard Gaussian measure (the proof in the discrete setting does not require anything new. We warn the reader that the proof in [46] contains an inaccurate duality argument). 
Lemma 5.4 (P.-A. Meyer). For every $p \in[2, \infty)$ there exists $c_{p} \in(0, \infty)$ such that for every $k \in \mathbb{N}$, every tail space function $f \in L_{p}^{\geqslant k}\left(\mathbb{F}_{2}^{n}\right)$ and every time $t \in(0, \infty)$,

$$
\left\|e^{-t \Delta} f\right\|_{L_{p}\left(\mathbb{F}_{2}^{n}\right)} \leqslant e^{-c_{p} k \min \left\{t, t^{2}\right\}}\|f\|_{L_{p}\left(\mathbb{F}_{2}^{n}\right)} .
$$

Hence,

$$
\|\Delta f\|_{L_{p}\left(\mathbb{F}_{2}^{n}\right)} \gtrsim c_{p} \sqrt{k} \cdot\|f\|_{L_{p}\left(\mathbb{F}_{2}^{n}\right)}
$$

Proof. The estimate (100) follows immediately from (99) as follows.

$$
\begin{aligned}
\|f\|_{L_{p}\left(\mathbb{F}_{2}^{n}\right)}=\| \int_{0}^{\infty} e^{-t \Delta} \Delta f d t & \|_{L_{p}\left(\mathbb{F}_{2}^{n}\right)} \\
& \leqslant \int_{0}^{\infty}\left\|e^{-t \Delta} \Delta f\right\|_{L_{p}\left(\mathbb{F}_{2}^{n}\right)} d t \\
& \stackrel{(999)}{\leqslant}\left(\int_{0}^{1} e^{-c_{p} k t^{2}} d t+\int_{1}^{\infty} e^{-c_{p} k t} d t\right)\|\Delta f\|_{L_{p}\left(\mathbb{F}_{2}^{n}\right)} \lesssim \frac{\|\Delta f\|_{L_{p}\left(\mathbb{F}_{2}^{n}\right)}}{c_{p} \sqrt{k}} .
\end{aligned}
$$

To prove (99), we may assume that $\|f\|_{L_{p}\left(\mathbb{F}_{2}^{n}\right)}=1$. Since $p \geqslant 2$, it follows that

$$
\left\|e^{-t \Delta} f\right\|_{L_{2}\left(\mathbb{F}_{2}^{n}\right)} \leqslant e^{-k t}\|f\|_{L_{2}\left(\mathbb{F}_{2}^{n}\right)} \leqslant e^{-k t}\|f\|_{L_{p}\left(\mathbb{F}_{2}^{n}\right)}=e^{-k t} .
$$

By classical hypercontractivity estimates [8, 7], if we define

$$
q \stackrel{\text { def }}{=} 1+e^{2 t}(p-1) \text {. }
$$

then

$$
\left\|e^{-t \Delta} f\right\|_{L_{q}\left(\mathbb{F}_{2}^{n}\right)} \leqslant\|f\|_{L_{p}\left(\mathbb{F}_{2}^{n}\right)}=1
$$

Since $p \in[2, q]$ we may consider $\theta \in[0,1]$ given by

$$
\frac{1}{p}=\frac{\theta}{2}+\frac{1-\theta}{q}
$$

Now,

$$
\begin{aligned}
\left\|e^{-t \Delta} f\right\|_{L_{p}\left(\mathbb{F}_{2}^{n}\right)} & \leqslant\left\|e^{-t \Delta} f\right\|_{L_{2}\left(\mathbb{F}_{2}^{n}\right)}^{\theta} \cdot\left\|e^{-t \Delta} f\right\|_{L_{q}\left(\mathbb{F}_{2}^{n}\right)}^{1-\theta} \\
\stackrel{(101) \wedge(103)}{\wedge} & e^{-k t \theta} \stackrel{(102) \wedge(104)}{=} \exp \left(-\frac{2(p-1) k t\left(e^{2 t}-1\right)}{p\left(e^{2 t}(p-1)-1\right)}\right) .
\end{aligned}
$$

By choosing $c_{p}$ appropriately, the desired estimate (99) is a consequence (105).

Remark 5.5. For the purpose of the geometric applications that are contained in the present paper we need to understand the vector-valued analogue of Lemma 5.4, i.e., Theorem [5.1, Nevertheless, The following interesting questions seem to be open for scalar-valued functions.

(1) Can one prove Lemma 5.4 also when $p \in(1,2)$ ? Note that while $\Delta$ and $e^{-t \Delta}$ are self-adjoint operators, one needs to understand the dual norm on $L_{p}^{\geqslant k}\left(\mathbb{F}_{2}^{n}, \mathbb{R}\right)^{*}$ in order to use duality here.

(2) What is the correct asymptotic dependence on $k$ in (100)? Specifically, can (100) be improved to

$$
\|\Delta f\|_{p} \gtrsim_{p} k\|f\|_{p} ?
$$

(3) As a potential way to prove (106), can one improve (99) to

$$
f \in L_{p}^{\geqslant k}\left(\mathbb{F}_{2}^{n}\right) \Longrightarrow \forall t \in(0, \infty), \quad\left\|e^{-t \Delta} f\right\|_{L_{p}\left(\mathbb{F}_{2}^{n}\right)} \leqslant e^{-c_{p} k t}\|f\|_{L_{p}\left(\mathbb{F}_{2}^{n}\right)} ?
$$


As some evidence for (107), P. Cattiaux proved (private communication) the case $k=1$, $p=4$ of (107) when the heat semigroup on $\mathbb{F}_{2}^{n}$ is replaced by the Ornstein-Uhlenbeck semigroup on $\mathbb{R}^{n}$. Specifically, let $\gamma_{n}$ be the standard Gaussian measure on $\mathbb{R}^{n}$ and consider the Ornstein-Uhlenbeck operator $L=\Delta-x \cdot \nabla$. Cattiaux proved that there exists a universal constant $c \in(0, \infty)$ such that for every $f \in L_{4}\left(\gamma_{n}, \mathbb{R}\right)$ and every $t \in(0, \infty)$,

$$
\int_{\mathbb{R}^{n}} f d \gamma_{n}=0 \Longrightarrow\left\|e^{-t L} f\right\|_{L_{4}\left(\gamma_{n}\right)} \leqslant e^{-c t}\|f\|_{L_{4}\left(\gamma_{n}\right)} \text {. }
$$

We shall now present a sketch of Cattiaux's proof of (108). By differentiating at $t=0$, integrating by parts, and using the semigroup property, one sees that (108) is equivalent to the following assertion.

$$
\int_{\mathbb{R}^{n}} f d \gamma_{n}=0 \Longrightarrow \int_{\mathbb{R}^{n}} f^{4} d \gamma_{n} \lesssim \int_{\mathbb{R}^{n}} f^{2}\|\nabla f\|_{2}^{2} d \gamma_{n}
$$

The Gaussian Poincaré inequality (see [9, 31]) applied to $f^{2}$ implies that

$$
\int_{\mathbb{R}^{n}} f^{4} d \gamma_{n}-\left(\int_{\mathbb{R}^{n}} f^{2} d \gamma_{n}\right)^{2} \lesssim \int_{\mathbb{R}^{n}} f^{2}\|\nabla f\|_{2}^{2} d \gamma_{n}
$$

The desired inequality (109) would therefore follow from

$$
\int_{\mathbb{R}^{n}} f d \gamma_{n}=0 \Longrightarrow\left(\int_{\mathbb{R}^{n}} f^{2} d \gamma_{n}\right)^{2} \lesssim \int_{\mathbb{R}^{n}} f^{2}\|\nabla f\|_{2}^{2} d \gamma_{n} .
$$

Fix $M \in(0, \infty)$ that will be determined later. Define $\phi_{M}: \mathbb{R} \rightarrow \mathbb{R}$ by

$$
\phi_{M}(x) \stackrel{\text { def }}{=} \begin{cases}0 & \text { if }|x| \leqslant M, \\ 2(x-M) & \text { if } x \in[M, 2 M], \\ 2(x+M) & \text { if } x \in[-2 M,-M], \\ x & \text { if }|x| \geqslant 2 M .\end{cases}
$$

Since $\left|\phi^{\prime}\right| \leqslant 2$, an application of the Gaussian Poincaré inequality to $\phi \circ f$ yields the estimate

$$
\int_{\mathbb{R}^{n}}(\phi \circ f)^{2} d \gamma_{n}-\left(\int_{\mathbb{R}^{n}} \phi \circ f d \gamma_{n}\right)^{2} \stackrel{(111)}{\lesssim} \int_{\{|f| \geqslant M\}}\|\nabla f\|_{2}^{2} d \gamma_{n} .
$$

Now,

$$
\int_{\mathbb{R}^{n}}(\phi \circ f)^{2} d \gamma_{n} \stackrel{\text { (111) }}{\geqslant} \int_{\{|f| \geqslant 2 M\}} f^{2} d \gamma_{n} \geqslant \int_{\mathbb{R}^{n}} f^{2} d \gamma_{n}-4 M^{2} .
$$

Also,

$$
\int_{\{|f| \geqslant M\}}\|\nabla f\|_{2}^{2} d \gamma_{n} \leqslant \frac{1}{M^{2}} \int_{\mathbb{R}^{n}} f^{2}\|\nabla f\|_{2}^{2} d \gamma_{n} .
$$

If in addition $\int_{\mathbb{R}^{n}} f d \gamma_{n}=0$ then

$$
\left|\int_{\mathbb{R}^{n}} \phi \circ f d \gamma_{n}\right|=\left|\int_{\mathbb{R}^{n}}(\phi \circ f-f) d \gamma_{n}\right| \stackrel{(111)}{=}\left|\int_{\{|f| \leqslant 2 M\}}(\phi \circ f-f) d \gamma_{n}\right| \leqslant 4 M .
$$

Hence, by (112), (113), (114) and (115),

$$
\int_{\mathbb{R}^{n}} f d \gamma_{n}=0 \Longrightarrow \int_{\mathbb{R}^{n}} f^{2} d \gamma_{n} \lesssim M^{2}+\frac{1}{M^{2}} \int_{\mathbb{R}^{n}} f^{2}\|\nabla f\|_{2}^{2} d \gamma_{n}
$$


The optimal choice of $M$ in (116) is

$$
M=\left(\int_{\mathbb{R}^{n}} f^{2}\|\nabla f\|_{2}^{2} d \gamma_{n}\right)^{1 / 4},
$$

yielding the desired inequality (110). It would be interesting to generalize the above argument so as to extend (108) to the setting of functions in all the Hermite tail spaces $\left\{L_{p}^{\geqslant k}\left(\gamma_{n}, \mathbb{R}\right)\right\}_{k \in \mathbb{N}}$ (i.e., functions whose Hermite coefficients of degree less than $k$ vanish).

5.2. Proof of Theorem 5.1. For every $m \in\{1, \ldots, n\}$ consider the level- $m$ Rademacher projection given by

$$
\operatorname{Rad}_{m}(f) \stackrel{\text { def }}{=} \sum_{\substack{A \subseteq\{1, \ldots, n\} \\|A|=m}} \widehat{f}(A) W_{A} .
$$

Thus $\boldsymbol{R a d}_{1}=\mathbf{R a d}$ and for every $z \in \mathbb{C}$ we have

$$
e^{z \Delta}=\sum_{m=0}^{n} e^{z m} \operatorname{Rad}_{m}
$$

We shall use the following deep theorem of Pisier [58].

Theorem 5.6 (Pisier). For every $K, p \in(1, \infty)$ there exist $\phi=\phi(K, p) \in(0, \pi / 4)$ and $M=M(K, p) \in(2, \infty)$ such that for every Banach space $X$ satisfying $K(X) \leqslant K, n \in \mathbb{N}$ and $z \in \mathbb{C}$, we have

$$
|\arg z| \leqslant \phi \Longrightarrow\left\|e^{-z \Delta}\right\|_{L_{p}\left(\mathbb{F}_{2}^{n}, X\right) \rightarrow L_{p}\left(\mathbb{F}_{2}^{n}, X\right)} \leqslant M .
$$

One can give explicit bounds on $M, \phi$ in terms of $p$ and $K$; see [40]. We will require the following standard corollary of Theorem 5.6. Define

$$
a=\frac{\pi}{\tan \phi},
$$

so that all the points in the open segment joining $a-i \pi$ and $a+i \pi$ have argument at most $\phi$. Then

$$
\left\|\operatorname{Rad}_{m}\right\|_{L_{p}\left(\mathbb{F}_{2}^{n}, X\right) \rightarrow L_{p}\left(\mathbb{F}_{2}^{n}, X\right)} \leqslant M e^{a m} .
$$

Indeed,

$$
\frac{1}{2 \pi} \int_{-\pi}^{\pi} e^{i m t} e^{-(a+i t) \Delta} d t=\frac{1}{2 \pi} \int_{-\pi}^{\pi} e^{i m t} \sum_{k=0}^{n} e^{-(a+i t) k} \mathbf{R a d}_{k} d t=e^{-m a} \mathbf{R a d}_{m} .
$$

Now (118) is deduced by convexity as follows.

$$
\left\|\operatorname{Rad}_{m}\right\|_{L_{p}\left(\mathbb{F}_{2}^{n}, X\right) \rightarrow L_{p}\left(\mathbb{F}_{2}^{n}, X\right)} \leqslant \frac{e^{m a}}{2 \pi} \int_{-\pi}^{\pi}\left\|e^{-(a+i t) \Delta}\right\|_{L_{p}\left(\mathbb{F}_{2}^{n}, X\right) \rightarrow L_{p}\left(\mathbb{F}_{2}^{n}, X\right)} d t \leqslant M e^{m a} .
$$

It follows that

$$
\Re z \geqslant 2 a \Longrightarrow\left\|e^{-z \Delta}\right\|_{L_{p}^{\geqslant k}\left(\mathbb{F}_{2}^{n}, X\right) \rightarrow L_{p}^{\geqslant k}\left(\mathbb{F}_{2}^{n}, X\right)} \leqslant \frac{M}{1-e^{-a}} e^{-k \Re z / 2} \leqslant \frac{M}{1-e^{-a}} e^{-k a} .
$$


Indeed,

$$
\begin{aligned}
\left\|e^{-z \Delta}\right\|_{L_{p}^{\geqslant k}}\left(\mathbb{F}_{2}^{n}, X\right) \rightarrow L_{p}^{\geqslant k}\left(\mathbb{F}_{2}^{n}, X\right) & \left\|\sum_{m=k}^{n} e^{-z m} \mathbf{R a d}_{m}\right\|_{L_{p}^{\geqslant k}\left(\mathbb{F}_{2}^{n}, X\right) \rightarrow L_{p}^{\geqslant k}\left(\mathbb{F}_{2}^{n}, X\right)} \\
& \stackrel{(118)}{\leqslant} \sum_{m=k}^{n} e^{-m \Re z} M e^{a m} \leqslant M \sum_{m=k}^{n} e^{-m \Re z / 2}=\frac{M}{1-e^{-\Re z / 2}} e^{-k \Re z / 2} \leqslant \frac{M}{1-e^{-a}} e^{-k \Re z / 2} .
\end{aligned}
$$

The ensuing argument is a quantitative variant of the proof of the main theorem of Pisier in 59]. Let

$$
r \stackrel{\text { def }}{=} 2 \sqrt{a^{2}+\pi^{2}}
$$

and define

$$
V \stackrel{\text { def }}{=}\{z \in \mathbb{C}:|z| \leqslant r \wedge|\arg z| \leqslant \phi\} .
$$

The set $V \subseteq \mathbb{C}$ is depicted in Figure 1.

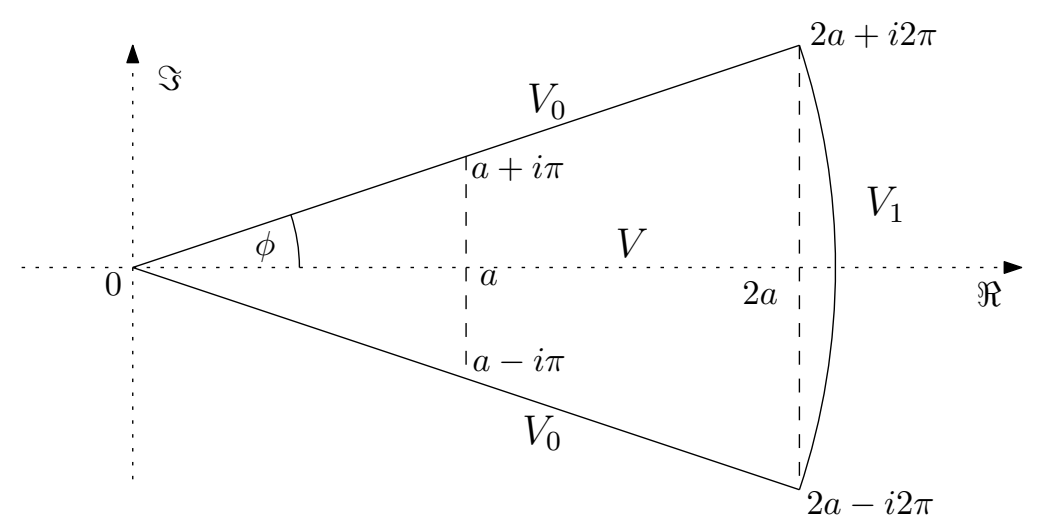

Figure 1. The sector $V \subseteq \mathbb{C}$.

Denote

$$
V_{0} \stackrel{\text { def }}{=}\{x \pm i x \tan \phi: x \in[0,2 a)\},
$$

and

$$
V_{1} \stackrel{\text { def }}{=}\left\{r e^{i \theta}:|\theta| \leqslant \phi\right\},
$$

so that we have the disjoint union $\partial V=V_{0} \cup V_{1}$.

Fix $t \in(0,2 a)$. Let $\mu_{t}$ be the harmonic measure corresponding to $V$ and $t$, i.e., $\mu_{t}$ is the Borel probability measure on $\partial V$ such that for every bounded analytic function $f: V \rightarrow \mathbb{C}$ we have

$$
f(t)=\int_{\partial V} f(z) d \mu_{t}(z)
$$

We refer to [16] for more information on this topic and the ensuing discussion. For concreteness, it suffices to recall here that for every Borel set $E \subseteq \partial V$ the number $\mu_{t}(E)$ is the probability that the standard 2-dimensional Brownian motion starting at $t$ exits $V$ at $E$. Equivalently, by conformal invariance, $\mu_{t}$ is the push-forward of the normalized Lebesgue measure on the unit circle $S^{1}$ under the Riemann mapping from the unit disk to $V$ which takes the origin to $t$. 
Denote

$$
\theta_{t} \stackrel{\text { def }}{=} \mu_{t}\left(V_{1}\right)
$$

and write

$$
\mu_{t}=\left(1-\theta_{t}\right) \mu_{t}^{0}+\theta_{t} \mu_{t}^{1}
$$

where $\mu_{t}^{0}, \mu_{t}^{1}$ are probability measures on $V_{0}, V_{1}$, respectively. We will use the following bound on $\theta_{t}$, whose proof is standard.

Lemma 5.7. For every $t \in(0,2 a)$ we have

$$
\theta_{t} \geqslant \frac{1}{2}\left(\frac{t}{r}\right)^{\frac{\pi}{2 \phi}}
$$

Proof. This is an exercise in conformal invariance. Let $\mathbb{D}=\{z \in \mathbb{C}:|z| \leqslant 1\}$ denote the unit disk centered at the origin, and let $\mathbb{D}_{+}$denote the intersection of $\mathbb{D}$ with the right half plane $\{z \in \mathbb{C}: \Re z \geqslant 0\}$. The mapping $h_{1}: V \rightarrow \mathbb{D}_{+}$given by

$$
h_{1}(z) \stackrel{\text { def }}{=}\left(\frac{z}{r}\right)^{\frac{\pi}{2 \phi}}
$$

is a conformal equivalence between $V$ and $\mathbb{D}_{+}$. Let $\mathbb{Q}_{+}=\{x+i y: x, y \in[0, \infty)\}$ denote the positive quadrant. The Möbius transformation $h_{2}: \mathbb{D}_{+} \rightarrow \mathbb{Q}_{+}$given by

$$
h_{2}(z) \stackrel{\text { def }}{=}-i \cdot \frac{z+i}{z-i}
$$

is a conformal equivalence between $\mathbb{D}_{+}$and $\mathbb{Q}_{+}$. The mapping $h_{3}(z) \stackrel{\text { def }}{=} z^{2}$ is a conformal equivalence between $\mathbb{Q}_{+}$and the upper half-plane $\mathbb{H}_{+}=\{z \in \mathbb{C}: \Im(z) \geqslant 0\}$. Finally, the Möbius transformation

$$
h_{4}(z) \stackrel{\text { def }}{=} \frac{z-i}{z+i}
$$

is a conformal equivalence between $\mathbb{H}_{+}$and $\mathbb{D}$. By composing these mappings, we obtain the following conformal equivalence between $V$ and $\mathbb{D}$.

$$
F(z) \stackrel{\text { def }}{=}\left(h_{4} \circ h_{3} \circ h_{2} \circ h_{1}\right)(z)=\frac{-\left(\left(\frac{z}{r}\right)^{\frac{\pi}{2 \phi}}+i\right)^{2}-i\left(\left(\frac{z}{r}\right)^{\frac{\pi}{2 \phi}}-i\right)^{2}}{-\left(\left(\frac{z}{r}\right)^{\frac{\pi}{2 \phi}}+i\right)^{2}+i\left(\left(\frac{z}{r}\right)^{\frac{\pi}{2 \phi}}-i\right)^{2}} .
$$

Therefore, the mapping $G: V \rightarrow \mathbb{D}$ given by

$$
G(z) \stackrel{\text { def }}{=} \frac{F(z)-F(t)}{1-\overline{F(t)} F(z)}
$$

is a conformal equivalence between $V$ and $\mathbb{D}$ with $G(t)=0$.

By conformal invariance, $\theta_{t}$ is the length of the arc $G\left(V_{1}\right) \subseteq \partial \mathbb{D}=S^{1}$, divided by $2 \pi$. Writing $s=h_{1}(t)=(t / r)^{\pi /(2 \phi)} \in(0,1)$, we have

$$
G(2 a+i 2 \pi)=\frac{-4 s\left(s^{2}-1\right)-i\left(\left(s^{2}-1\right)^{2}-4 s^{2}\right)}{\left(s^{2}+1\right)^{2}},
$$

and

$$
G(2 a-i 2 \pi)=\frac{4 s\left(s^{2}-1\right)-i\left(\left(s^{2}-1\right)^{2}-4 s^{2}\right)}{\left(s^{2}+1\right)^{2}} .
$$


It follows that if $s \geqslant \sqrt{2}-1$ then $\theta_{t} \geqslant \frac{1}{2}$, and if $s<\sqrt{2}-1$ then

$$
\theta_{t}=\frac{1}{\pi} \arcsin \left(\frac{4 s\left(1-s^{2}\right)}{\left(s^{2}+1\right)^{2}}\right) \geqslant \frac{s}{2}
$$

where the rightmost inequality in (123) follows from elementary calculus.

Lemma 5.8. For every $\varepsilon \in(0,1)$ there exists a bounded analytic function $\Psi_{\varepsilon}^{t}: V \rightarrow \mathbb{C}$ satisfying

- $\Psi_{\varepsilon}^{t}(t)=1$,

- $\left|\Psi_{\varepsilon}^{t}(z)\right|=\varepsilon$ for every $z \in V_{0}$,

- $\left|\Psi_{\varepsilon}^{t}(z)\right|=\frac{1}{\varepsilon^{\left(1-\theta_{t}\right) / \theta_{t}}}$ for every $z \in V_{1}$.

Proof. The proof is the same as the proof of Claim 2 in [59]. We sketch it briefly for the sake of completeness. Consider the strip $S=\{z \in \mathbb{C}: \Re(z) \in[0,1]\}$ and for $j \in\{0,1\}$ let $S_{j}=\{z \in \mathbb{C}: \Re(z)=j\}$. As explained in [59, Claim 1], there exists a conformal equivalence $h: V \rightarrow S$ such that $h(t)=\theta_{t}, h\left(V_{0}\right)=S_{0}$ and $h\left(V_{1}\right)=S_{1}$. Now define $\Psi_{\varepsilon}(z) \stackrel{\text { def }}{=} \varepsilon^{1-\frac{h(z)}{\theta_{t}}}$.

Proof of Theorem 5.1. Take $t \in(0, \infty)$. If $t \geqslant 2 a$ then by (119) we have

$$
\left\|e^{-t \Delta}\right\|_{L_{p}^{\geqslant k}\left(\mathbb{F}_{2}^{n}, X\right) \rightarrow L_{p}^{\geqslant k}\left(\mathbb{F}_{2}^{n}, X\right)} \leqslant \frac{M}{1-e^{-a}} e^{-k t / 2} .
$$

Suppose therefore that $t \in(0,2 a)$. Fix $\varepsilon \in(0,1)$ that will be determined later, and let $\Psi_{\varepsilon}^{t}$ be the function from Lemma 5.8. Then

$$
\begin{aligned}
e^{-t \Delta}=\Psi_{\varepsilon}^{t}(t) e^{-t \Delta} \stackrel{\frac{(120)}{=}}{=} \int_{\partial V} \Psi_{\varepsilon}^{t}(z) e^{-z \Delta} d \mu_{t}(z) & \\
& \stackrel{(121)}{=}\left(1-\theta_{t}\right) \int_{V_{0}} \Psi_{\varepsilon}^{t}(z) e^{-z \Delta} d \mu_{t}^{0}(z)+\theta_{t} \int_{V_{1}} \Psi_{\varepsilon}^{t}(z) e^{-z \Delta} d \mu_{t}^{1}(z) .
\end{aligned}
$$

Hence, using (125) in combination with Lemma 5.8, Theorem 5.6 and (119), we deduce that

$$
\begin{aligned}
\left\|e^{-t \Delta}\right\|_{L_{p}^{\geqslant k}\left(\mathbb{F}_{2}^{n}, X\right) \rightarrow L_{p}^{\geqslant k}\left(\mathbb{F}_{2}^{n}, X\right)} \leqslant\left(1-\theta_{t}\right) \varepsilon M+\frac{\theta_{t}}{\varepsilon^{\left(1-\theta_{t}\right) / \theta_{t}}} \cdot \frac{M e^{-k a}}{1-e^{-a}} & \stackrel{(122)}{\leqslant} \varepsilon M+\frac{M e^{-k a}}{1-e^{-a}} \cdot \frac{1}{\varepsilon^{2(r / t)^{\pi /(2 \phi)}-1}} .
\end{aligned}
$$

We now choose

$$
\varepsilon=\exp \left(-\frac{1}{2}\left(\frac{t}{r}\right)^{\frac{\pi}{2 \phi}} k a\right),
$$

in which case (126) completes the proof of Theorem 5.1, with $B(K, p)=\frac{\pi}{2 \phi}$.

5.3. Proof of Theorem $\mathbf{5 . 2}$. The elementary computation contained in Lemma 5.9 below will be useful in ensuing considerations.

Lemma 5.9. Define $f_{n}: \mathbb{F}_{2}^{n} \rightarrow L_{1}\left(\mathbb{F}_{2}^{n}\right)$ by

$$
f_{n}(x)(y)=2^{n} \mathbf{1}_{\{x=y\}}-1 .
$$


Then $f_{n} \in L_{p}^{\geqslant 1}\left(\mathbb{F}_{2}^{n}, L_{1}\left(\mathbb{F}_{2}^{n}\right)\right)$, yet for every $t \in(0, \infty)$ we have

$$
\lim _{n \rightarrow \infty} \frac{\left\|e^{-t \Delta} f_{n}\right\|_{L_{p}\left(\mathbb{F}_{2}^{n}, L_{1}\left(\mathbb{F}_{2}^{n}\right)\right)}}{\left\|f_{n}\right\|_{L_{p}\left(\mathbb{F}_{2}^{n}, L_{1}\left(\mathbb{F}_{2}^{n}\right)\right)}}=1
$$

where the limit in (128) is uniform in $p \in[1, \infty)$.

Proof. By definition $\sum_{x \in \mathbb{F}_{2}^{n}} f_{n}(x)=0$, i.e., $f_{n} \in L_{p}^{\geqslant 1}\left(\mathbb{F}_{2}^{n}, L_{1}\left(\mathbb{F}_{2}^{n}\right)\right)$. Observe that

$$
\left\|f_{n}\right\|_{L_{p}\left(\mathbb{F}_{2}^{n}, L_{1}\left(\mathbb{F}_{2}^{n}\right)\right)}=2\left(1-\frac{1}{2^{n}}\right),
$$

and note also that for every $x, y \in \mathbb{F}_{2}^{n}$ we have

$$
f_{n}(x)(y)=\prod_{i=1}^{n}\left(1+(-1)^{x_{i}+y_{i}}\right)-1=\sum_{\substack{A \subseteq\{1, \ldots, n\} \\ A \neq \emptyset}} W_{A}(x) W_{A}(y) .
$$

It follows from (95) that for every $x \in \mathbb{F}_{2}^{n}$ we have

$$
\begin{aligned}
\left\|e^{-t \Delta} f_{n}\right\|_{L_{1}\left(\mathbb{F}_{2}^{n}\right)}=\frac{1}{2^{n}} \sum_{y \in \mathbb{F}_{2}^{n}}\left|\sum_{w \in \mathbb{F}_{2}^{n}}\left(\frac{1-e^{t}}{2}\right)^{\|x-w\|_{1}}\left(\frac{1+e^{t}}{2}\right)^{n-\|x-w\|_{1}} f_{n}(w)(y)\right| \\
\stackrel{(127)}{=} \frac{1}{2^{n}} \sum_{y \in \mathbb{F}_{2}^{n}}\left|2^{n}\left(\frac{1-e^{t}}{2}\right)^{\|x-y\|_{1}}\left(\frac{1+e^{t}}{2}\right)^{n-\|x-y\|_{1}}-1\right| .
\end{aligned}
$$

Hence,

$$
\left\|e^{-t \Delta} f_{n}\right\|_{L_{p}\left(\mathbb{F}_{2}^{n}, L_{1}\left(\mathbb{F}_{2}^{n}\right)\right)}=\sum_{m=0}^{n}\left(\begin{array}{c}
n \\
m
\end{array}\right)\left|\left(\frac{1-e^{-t}}{2}\right)^{m}\left(\frac{1+e^{-t}}{2}\right)^{n-m}-\frac{1}{2^{n}}\right| .
$$

Let $U_{1}, \ldots, U_{n}$ be i.i.d. random variables such that $\operatorname{Pr}\left[U_{1}=0\right]=\operatorname{Pr}\left[U_{1}=1\right]=\frac{1}{2}$. By the Central Limit Theorem,

$$
\begin{aligned}
1=\lim _{n \rightarrow \infty} \operatorname{Pr}\left[\sum_{j=1}^{n} U_{j} \in\left(\frac{1}{2} n-n^{2 / 3}, \frac{1}{2} n+n^{2 / 3}\right)\right] & \\
& =\lim _{n \rightarrow \infty} \sum_{m \in\left(\frac{1}{2} n-n^{2 / 3}, \frac{1}{2} n+n^{2 / 3}\right) \cap \mathbb{N}}\left(\begin{array}{c}
n \\
m
\end{array}\right) \frac{1}{2^{n}} .
\end{aligned}
$$

Similarly, if $V_{1}, \ldots, V_{n}$ are i.i.d. random variables such that $\operatorname{Pr}\left[V_{1}=1\right]=\left(1-e^{-t}\right) / 2$ and $\operatorname{Pr}\left[V_{1}=0\right]=\left(1+e^{-t}\right) / 2$, then by the Central Limit Theorem,

$$
\begin{aligned}
1=\lim _{n \rightarrow \infty} \operatorname{Pr} & {\left[\sum_{j=1}^{n} V_{j} \in\left(\frac{1-e^{-t}}{2} n-n^{2 / 3}, \frac{1-e^{-t}}{2} n+n^{2 / 3}\right)\right] } \\
& =\lim _{n \rightarrow \infty} \sum_{m \in\left(\frac{1-e^{-t}}{2} n-n^{2 / 3}, \frac{1-e^{-t}}{2} n+n^{2 / 3}\right) \cap \mathbb{N}}\left(\begin{array}{c}
n \\
m
\end{array}\right)\left(\frac{1-e^{-t}}{2}\right)^{m}\left(\frac{1+e^{-t}}{2}\right)^{n-m} .
\end{aligned}
$$


Fix $\varepsilon \in(0,1)$. It follows from (132), (133) that for $n$ large enough we have

$$
\sum_{m \in\left(\frac{1}{2} n-n^{2 / 3}, \frac{1}{2} n+n^{2 / 3}\right) \cap \mathbb{N}}\left(\begin{array}{c}
n \\
m
\end{array}\right) \frac{1}{2^{n}} \geqslant 1-\frac{\varepsilon}{2},
$$

and

$$
\sum_{m \in\left(\frac{1-e^{-t}}{2} n-n^{2 / 3}, \frac{1-e^{-t}}{2} n+n^{2 / 3}\right) \cap \mathbb{N}}\left(\begin{array}{c}
n \\
m
\end{array}\right)\left(\frac{1-e^{-t}}{2}\right)^{m}\left(\frac{1+e^{-t}}{2}\right)^{n-m} \geqslant 1-\frac{\varepsilon}{2} .
$$

Moreover, by choosing $n$ to be large enough we can ensure that

$$
\left(\frac{1}{2} n-n^{2 / 3}, \frac{1}{2} n+n^{2 / 3}\right) \cap\left(\frac{1-e^{-t}}{2} n-n^{2 / 3}, \frac{1-e^{-t}}{2} n+n^{2 / 3}\right)=\emptyset .
$$

Since

$$
\begin{aligned}
m \in\left(\frac{1}{2} n-n^{2 / 3}, \frac{1}{2} n+n^{2 / 3}\right) & \Longrightarrow \\
& \left(\frac{1-e^{-t}}{2}\right)^{m}\left(\frac{1+e^{-t}}{2}\right)^{n-m}<\frac{1}{2^{n}}\left(1-e^{-2 t}\right)^{n / 2}\left(\frac{1+e^{-t}}{1-e^{-t}}\right)^{n^{2 / 3}},
\end{aligned}
$$

if $n$ is large enough then

$$
m \in\left(\frac{1}{2} n-n^{2 / 3}, \frac{1}{2} n+n^{2 / 3}\right) \Longrightarrow\left(\frac{1-e^{-t}}{2}\right)^{m}\left(\frac{1+e^{-t}}{2}\right)^{n-m}<\frac{\varepsilon}{2^{n+1}} .
$$

Moreover, because $t>0$ we have $h\left(\left(1-e^{-t}\right) / 2\right)>\frac{1}{2}$, where $h(s) \stackrel{\text { def }}{=} s^{s}(1-s)^{1-s}$ for $s \in[0,1]$. Noting that

$$
\begin{aligned}
m \in\left(\frac{1-e^{-t}}{2} n-n^{2 / 3},\right. & \left.\frac{1-e^{-t}}{2} n+n^{2 / 3}\right) \Longrightarrow \\
& \left(\frac{1-e^{-t}}{2}\right)^{m}\left(\frac{1+e^{-t}}{2}\right)^{n-m}>\left(h\left(\frac{1-e^{-t}}{2}\right)\right)^{n}\left(\frac{1-e^{-t}}{1+e^{-t}}\right)^{n^{2 / 3}},
\end{aligned}
$$

we see that if $n$ is large enough then

$$
m \in\left(\frac{1-e^{-t}}{2} n-n^{2 / 3}, \frac{1-e^{-t}}{2} n+n^{2 / 3}\right) \Longrightarrow \frac{1}{2^{n}}<\frac{\varepsilon}{2}\left(\frac{1-e^{-t}}{2}\right)^{m}\left(\frac{1+e^{-t}}{2}\right)^{n-m} .
$$

Consequently, if we choose $n$ so as to ensure the validity of (134), (135), (136), (137), (138), then recalling (129) we see that

$$
\left\|e^{-t \Delta} f_{n}\right\|_{L_{p}\left(\mathbb{F}_{2}^{n}, L_{1}\left(\mathbb{F}_{2}^{n}\right)\right)} \geqslant 2\left(1-\frac{\varepsilon}{2}\right)^{2} \stackrel{(129)}{\geqslant}(1-\varepsilon)\left\|f_{n}\right\|_{L_{p}\left(\mathbb{F}_{2}^{n}, L_{1}\left(\mathbb{F}_{2}^{n}\right)\right)}
$$

Proof of Theorem 5.2. Suppose that there exists $\delta \in(0,1), k \in \mathbb{N}, p \in(1, \infty)$ and $t \in(0, \infty)$ such that

$$
\forall n \in \mathbb{N}, \quad\left\|e^{-t \Delta}\right\|_{L_{p}^{\geqslant k}\left(\mathbb{F}_{2}^{n}, X\right) \rightarrow L_{p}^{\geqslant k}\left(\mathbb{F}_{2}^{n}, X\right)}<1-\delta .
$$


For $n \in \mathbb{N}$, identify $\mathbb{F}_{2}^{k n}$ with the $k$-fold product of $\mathbb{F}_{2}^{n}$. Define $F: \mathbb{F}_{2}^{k n} \rightarrow L_{1}\left(\mathbb{F}_{2}^{k n}\right)$ by

$$
F\left(x^{1}, \ldots, x^{k}\right)\left(y^{1}, \ldots, y^{k}\right) \stackrel{\text { def }}{=} \prod_{i=1}^{k} f_{n}\left(x^{i}\right)\left(y^{i}\right),
$$

where $f_{n} \in L_{p}^{\geqslant 1}\left(\mathbb{F}_{2}^{n}, L_{1}\left(\mathbb{F}_{2}^{n}\right)\right)$ is given in (127). Then $F \in L_{p}^{\geqslant k}\left(\mathbb{F}_{2}^{k n}, L_{1}\left(\mathbb{F}_{2}^{k n}\right)\right)$. For every injective linear operator $T: L_{1}\left(\mathbb{F}_{2}^{k n}\right) \rightarrow X$ we have $T \circ F \in L_{p}^{\geqslant k}\left(\mathbb{F}_{2}^{k n}, X\right)$, and therefore

$$
\begin{aligned}
1-\delta \stackrel{(139)}{>} \frac{\left\|e^{-t \Delta}(T \circ F)\right\|_{L_{p}\left(\mathbb{F}_{2}^{k n}, X\right)}}{\|T \circ F\|_{L_{p}\left(\mathbb{F}_{2}^{k n}, X\right)}} \geqslant \frac{1}{\|T\| \cdot\left\|T^{-1}\right\|} \cdot \frac{\left\|e^{-t \Delta} F\right\|_{L_{p}\left(\mathbb{F}_{2}^{k n}, L_{1}\left(\mathbb{F}_{2}^{k n}\right)\right)}}{\|F\|_{L_{p}\left(\mathbb{F}_{2}^{k n}, L_{1}\left(\mathbb{F}_{2}^{k n}\right)\right)}} \\
\stackrel{(140)}{=} \frac{1}{\|T\| \cdot\left\|T^{-1}\right\|} \cdot\left(\frac{\left\|e^{-t \Delta} f_{n}\right\|_{L_{p}\left(\mathbb{F}_{2}^{n}, L_{1}\left(\mathbb{F}_{2}^{n}\right)\right)}}{\left\|f_{n}\right\|_{L_{p}\left(\mathbb{F}_{2}^{n}, L_{1}\left(\mathbb{F}_{2}^{n}\right)\right)}}\right)^{k} \underset{n \rightarrow \infty}{\longrightarrow} \frac{1}{\|T\| \cdot\left\|T^{-1}\right\|},
\end{aligned}
$$

where in the last step of (141) we used Lemma 5.9. It follows that

$$
\sup _{m \in \mathbb{N}} \inf _{S \in \mathscr{L}\left(\ell_{1}^{m}, X\right)}\|S\| \cdot\left\|S^{-1}\right\| \geqslant \frac{1}{1-\delta} .
$$

By Pisier's $K$-convexity theorem [58] we conclude that $X$ must be $K$-convex.

5.4. Inverting the Laplacian on the vector-valued tail space. Here we discuss lower bounds on the restriction of $\Delta$ to the tail space. Such bounds can potentially yield a simplification of our construction of the base graph; see Remarks 5.12 and 7.5 below.

Theorem 5.10. For every $K, p \in(1, \infty)$ there exist $\delta=\delta(K, p), c=c(K, p) \in(0,1)$ such that if $X$ is a $K$-convex Banach space with $K(X) \leqslant K$ then for every $n \in \mathbb{N}$ and $k \in\{1, \ldots, n\}$,

$$
f \in L_{p}^{\geqslant k}\left(\mathbb{F}_{2}^{n}, X\right) \Longrightarrow\|\Delta f\|_{L_{p}\left(\mathbb{F}_{2}^{n}, X\right)} \geqslant c k^{\delta} \cdot\|f\|_{L_{p}\left(\mathbb{F}_{2}^{n}, X\right)}
$$

Proof. The estimate (142) is deduced from Theorem 5.1 as follows. If $f \in L_{p}^{\geqslant k}\left(\mathbb{F}_{2}^{n}, X\right)$ then

$$
\begin{gathered}
\|f\|_{L_{p}\left(\mathbb{F}_{2}^{n}, X\right)}=\left\|\int_{0}^{\infty} e^{-t \Delta} \Delta f d t\right\|_{L_{p}\left(\mathbb{F}_{2}^{n}, X\right)} \stackrel{\sqrt[97]{7}}{\leqslant} C\left(\int_{0}^{1} e^{-A k t^{B}} d t+\int_{1}^{\infty} e^{-A k t} d t\right)\|\Delta f\|_{L_{p}\left(\mathbb{F}_{2}^{n}, X\right)} \\
\leqslant C\left(\frac{\Gamma(1 / B)}{(A k)^{1 / B}}+\frac{e^{-A k}}{A k}\right)\|\Delta f\|_{L_{p}\left(\mathbb{F}_{2}^{n}, X\right)} \lesssim \frac{C B}{A^{1 / B}} \cdot \frac{1}{k^{1 / B}}\|\Delta f\|_{L_{p}\left(\mathbb{F}_{2}^{n}, X\right)} .
\end{gathered}
$$

We also have the following converse to Theorem 5.10 ,

Theorem 5.11. If $X$ is a Banach space such that for some $p, K \in(0, \infty)$ and $k \in \mathbb{N}$ we have

$$
\lim _{n \rightarrow \infty} \inf _{\substack{f \in L_{p}^{\geqslant k}\left(\mathbb{F}_{2}^{n}, X\right) \\ f \neq 0}} \frac{\|\Delta f\|_{L_{p}\left(\mathbb{F}_{2}^{n}, X\right)}}{\|f\|_{L_{p}\left(\mathbb{F}_{2}^{n}, X\right)}}>0,
$$

then $X$ is $K$-convex.

Proof. For $f \in L_{p}\left(\mathbb{F}_{2}^{n}, X\right)$ define

$$
\Delta^{-1} f=\sum_{\substack{A \subseteq\{1, \ldots, n\} \\ A \neq \emptyset}} \frac{1}{|A|} \widehat{f}(A) W_{A} .
$$


In [52, Thm. 5] it was shown that if $X$ is not $K$-convex then

$$
\sup _{n \in \mathbb{N}}\left\|\Delta^{-1}\right\|_{L_{p}\left(\mathbb{F}_{2}^{n}, X\right) \rightarrow L_{p}\left(\mathbb{F}_{2}^{n}, X\right)}=\infty .
$$

Here we need to extend this statement to the assertion contained in (144) below, which should hold true for every Banach space $X$ that is not $K$-convex and every $k \in \mathbb{N}$.

$$
\sup _{n \in \mathbb{N}}\left\|\Delta^{-1}\right\|_{L_{p}^{\geqslant k}\left(\mathbb{F}_{2}^{n}, X\right) \rightarrow L_{p}^{\geqslant k}\left(\mathbb{F}_{2}^{n}, X\right)}=\infty
$$

Arguing as in the proof of Theorem 5.2, by Pisier's $K$-convexity theorem [58] it will suffice to prove that for $n \geqslant 2$, if $F: \mathbb{F}_{2}^{k n} \rightarrow L_{1}\left(F_{2}^{k n}\right)$ is given as in (140) then

$$
\frac{\left\|\Delta^{-1} F\right\|_{L_{p}\left(\mathbb{F}_{2}^{k n}, L_{1}\left(\mathbb{F}_{2}^{k n}\right)\right)}}{\|F\|_{L_{p}\left(\mathbb{F}_{2}^{k n}, L_{1}\left(\mathbb{F}_{2}^{k n}\right)\right)}} \gtrsim \frac{\log n}{8^{k}}
$$

Note that by (129),

$$
\|F\|_{L_{p}\left(\mathbb{F}_{2}^{k n}, L_{1}\left(\mathbb{F}_{2}^{k n}\right)\right)}=2^{k}\left(1-\frac{1}{2^{n}}\right)^{k} \leqslant 2^{k} .
$$

By (130) and (140), for every $\left(x^{1}, \ldots, x^{k}\right),\left(y^{1}, \ldots, y^{k}\right) \in \mathbb{F}_{2}^{k n}$ and every $t \in(0, \infty)$,

$$
\begin{aligned}
e^{-t \Delta} F\left(x^{1}, \ldots, x^{k}\right)\left(y^{1}, \ldots, y^{k}\right)= & \prod_{i=1}^{k}\left(\prod_{j=1}^{n}\left(1+e^{-t}(-1)^{x_{j}^{i}+y_{j}^{i}}\right)-1\right) \\
& =\prod_{i=1}^{k}\left(\left(1-e^{-t}\right)^{\left\|x^{i}-y^{i}\right\|_{1}}\left(1+e^{-t}\right)^{n-\left\|x^{i}-y^{i}\right\|_{1}}-1\right) .
\end{aligned}
$$

For every $x \in \mathbb{F}_{2}^{n}$ denote

$$
\Omega_{x} \stackrel{\text { def }}{=}\left\{y \in \mathbb{F}_{2}^{n}:\|y-x\|_{1} \leqslant \frac{n}{2}\right\}
$$

Then

$$
\forall x \in \mathbb{F}_{2}^{n}, \quad\left|\Omega_{x}\right| \geqslant 2^{n-1},
$$

and by (147) we have

$$
\left(y^{1}, \ldots, y^{k}\right) \in \prod_{i=1}^{k} \Omega_{x^{i}} \Longrightarrow\left|e^{-t \Delta} F\left(x^{1}, \ldots, x^{k}\right)\left(y^{1}, \ldots, y^{k}\right)\right| \geqslant\left(1-\left(1-e^{-2 t}\right)^{n / 2}\right)^{k}
$$

Now,

$$
\begin{aligned}
\left\|\Delta^{-1} F\left(x^{1}, \ldots, x^{k}\right)\right\|_{L_{1}\left(\mathbb{F}_{2}^{k n}\right)} & =\left\|\int_{0}^{\infty} e^{-t \Delta} F\left(x^{1}, \ldots, x^{k}\right) d t\right\|_{L_{1}\left(\mathbb{F}_{2}^{k n}\right)} \\
& \geqslant \frac{1}{2^{k n}} \sum_{\left(y^{1}, \ldots, y^{k}\right) \in \prod_{i=1}^{k} \Omega_{x^{i}}}\left|\int_{0}^{\infty} e^{-t \Delta} F\left(x^{1}, \ldots, x^{k}\right)\left(y^{1}, \ldots, y^{k}\right) d t\right| \\
& \geqslant \frac{1}{2^{k}} \int_{0}^{\infty}\left(1-\left(1-e^{-2 t}\right)^{n / 2}\right)^{k} d t
\end{aligned}
$$

where in (150) we used (148) and (149). Finally,

$$
\left\|\Delta^{-1} F\right\|_{L_{p}\left(\mathbb{F}_{2}^{k n}, L_{1}\left(\mathbb{F}_{2}^{k n}\right)\right)} \stackrel{(150)}{\geqslant} \frac{1}{2^{k}} \int_{0}^{\frac{1}{2} \log n}\left(1-\left(1-e^{-2 t}\right)^{n / 2}\right)^{k} d t \gtrsim \frac{\log n}{4^{k}} .
$$


The desired estimate (145) now follows from (146) and (151).

Remark 5.12. The following natural problem presents itself. Can one improve Theorem 5.10 so as to have $\delta=1$, i.e., to obtain the bound

$$
f \in L_{p}^{\geqslant k}\left(\mathbb{F}_{2}^{n}, X\right) \Longrightarrow\|\Delta f\|_{L_{p}\left(\mathbb{F}_{2}^{n}, X\right)} \geqslant c(K, p) k \cdot\|f\|_{L_{p}\left(\mathbb{F}_{2}^{n}, X\right)} ?
$$

As discussed in Remark 5.5, this seems to be unknown even when $X=\mathbb{R}$. If (152) were true then it would significantly simplify our construction of the base graph, since in Section 7 we would be able to use the "vanilla" hypercube quotients of [27] instead of the quotients of the discretized heat semigroup as in Lemma 7.3, see Remark 7.5 below for more information on this potential simplification.

\section{Nonlinear sPeCtral Gaps In UNIFORMly CONVEX NORMED SPACES}

Let $\left(X,\|\cdot\|_{X}\right)$ be a normed space. For $n \in \mathbb{N}$ and $p \in[1, \infty)$ we let $L_{p}^{n}(X)$ denote the space of functions $f:\{1, \ldots, n\} \rightarrow X$, equipped with the norm

$$
\|f\|_{L_{p}^{n}(X)}=\left(\frac{1}{n} \sum_{i=1}^{n}\|f(i)\|_{X}^{p}\right)^{1 / p}
$$

Thus, using the notation introduced in the beginning of Section $5, L_{p}^{n}(X)=L_{p}^{n}(\{1, \ldots, n\}, X)$. We shall also use the notation

$$
L_{p}^{n}(X)_{0} \stackrel{\text { def }}{=}\left\{f \in L_{p}^{n}(X): \sum_{i=1}^{n} f(i)=0\right\} .
$$

Given an $n \times n$ symmetric stochastic matrix $A=\left(a_{i j}\right)$ we denote by $A \otimes I_{X}^{n}$ the operator from $L_{p}^{n}(X)$ to $L_{p}^{n}(X)$ given by

$$
\left(A \otimes I_{X}^{n}\right) f(i)=\sum_{j=1}^{n} a_{i j} f(j) .
$$

Note that since $A$ is symmetric and stochastic the operator $A \otimes I_{X}^{n}$ preserves the subspace $L_{p}^{n}(X)_{0}$, that is $\left(A \otimes I_{X}^{n}\right)\left(L_{p}^{n}(X)_{0}\right) \subseteq L_{p}^{n}(X)_{0}$. Define

$$
\lambda_{X}^{(p)}(A) \stackrel{\text { def }}{=}\left\|A \otimes I_{X}^{n}\right\|_{L_{p}^{n}(X)_{0} \rightarrow L_{p}^{n}(X)_{0}} .
$$

Note that, since $A$ is doubly stochastic, $\lambda_{X}^{p}(A) \leqslant 1$. It is immediate to check that

$$
\lambda_{\mathbb{R}}^{(2)}(A)=\lambda_{L_{2}}^{(2)}(A)=\lambda(A)=\max _{i \in\{2, \ldots, n\}}\left|\lambda_{i}(A)\right| .
$$

Thus $\lambda_{X}^{(p)}(A)$ should be viewed as a non-Euclidean (though still linear) variant of the absolute spectral gap of $A$. The following lemma substantiates this analogy by establishing a relation between $\lambda_{X}^{(p)}(A)$ and $\gamma_{+}\left(A,\|\cdot\|_{X}^{p}\right)$.

Lemma 6.1. For every normed space $\left(X,\|\cdot\|_{X}\right)$, every $p \geqslant 1$ and every $n \times n$ symmetric stochastic matrix A, we have

$$
\gamma_{+}\left(A,\|\cdot\|_{X}^{p}\right) \leqslant\left(1+\frac{4}{1-\lambda_{X}^{(p)}(A)}\right)^{p} .
$$


Proof. Write $\lambda=\lambda_{X}^{(p)}(A)$. We may assume that $\lambda<1$, since otherwise there is nothing to prove. Fix $f, g:\{1, \ldots, n\} \rightarrow X$ and denote

$$
\bar{f} \stackrel{\text { def }}{=} \frac{1}{n} \sum_{i=1}^{n} f(i) \quad \text { and } \quad \bar{g} \stackrel{\text { def }}{=} \frac{1}{n} \sum_{i=1}^{n} g(i) .
$$

Thus

$$
f_{0} \stackrel{\text { def }}{=} f-\bar{f} \in L_{p}^{n}(X)_{0} \quad \text { and } \quad g_{0} \stackrel{\text { def }}{=} g-\bar{g} \in L_{p}^{n}(X)_{0} .
$$

Therefore

$$
\left\|\left(A \otimes I_{X}^{n}\right) f_{0}\right\|_{L_{p}^{n}(X)} \leqslant \lambda\left\|f_{0}\right\|_{L_{p}^{n}(X)} \quad \text { and } \quad\left\|\left(A \otimes I_{X}^{n}\right) g_{0}\right\|_{L_{p}^{n}(X)} \leqslant \lambda\left\|g_{0}\right\|_{L_{p}^{n}(X)}
$$

Let $B$ be the $(2 n) \times(2 n)$ symmetric stochastic matrix given by

$$
B=\left(\begin{array}{cc}
0 & A \\
A & 0
\end{array}\right)
$$

Letting $h=f_{0} \oplus g_{0} \in L_{p}^{2 n}(X)$ be given by

$$
h(i) \stackrel{\text { def }}{=} \begin{cases}f_{0}(i) & \text { if } i \in\{1, \ldots, n\}, \\ g_{0}(i-n) & \text { if } i \in\{n+1, \ldots, 2 n\},\end{cases}
$$

we see that

$$
\begin{aligned}
(1-\lambda) & \|h\|_{L_{p}^{2 n}(X)}=\|h\|_{L_{p}^{2 n}(X)}-\left(\frac{\lambda^{p}\left\|f_{0}\right\|_{L_{p}^{n}(X)}^{p}+\lambda^{p}\left\|g_{0}\right\|_{L_{p}^{n}(X)}^{p}}{2}\right)^{1 / p} \\
& \stackrel{(155)}{\leqslant}\|h\|_{L_{p}^{2 n}(X)}-\left(\frac{1}{2}\left\|\left(A \otimes I_{X}^{n}\right) f_{0}\right\|_{L_{p}^{n}(X)}^{p}+\frac{1}{2}\left\|\left(A \otimes I_{X}^{n}\right) g_{0}\right\|_{L_{p}^{n}(X)}^{p}\right)^{1 / p} \\
& \stackrel{(156)}{=}\|h\|_{L_{p}^{2 n}(X)}-\left\|\left(B \otimes I_{X}^{2 n}\right) h\right\|_{L_{p}^{2 n}(X)} \\
& \leqslant\left\|\left(I_{L_{p}^{2 n}(X)}-B \otimes I_{X}^{2 n}\right) h\right\|_{L_{p}^{2 n}(X)} \\
& =\left(\frac{1}{2 n} \sum_{i=1}^{n}\left\|\sum_{j=1}^{n} a_{i j}\left(f_{0}(i)-g_{0}(j)\right)\right\|_{X}^{p}+\frac{1}{2 n} \sum_{i=1}^{n}\left\|\sum_{j=1}^{n} a_{i j}\left(g_{0}(i)-f_{0}(j)\right)\right\|_{X}^{p}\right)^{1 / p} \\
& \leqslant\left(\frac{1}{n} \sum_{i=1}^{n} \sum_{j=1}^{n} a_{i j}\left\|f_{0}(i)-g_{0}(j)\right\|_{X}^{p}\right)^{1 / p} \\
& \leqslant\|\bar{f}-\bar{g}\|_{X}+\left(\frac{1}{n} \sum_{i=1}^{n} \sum_{j=1}^{n} a_{i j}\|f(i)-g(j)\|_{X}^{p}\right)^{1 / p} \cdot
\end{aligned}
$$


Note that

$$
\begin{aligned}
\|\bar{f}-\bar{g}\|_{X}= & \left\|\frac{1}{n} \sum_{i=1}^{n} \sum_{j=1}^{n} a_{i j}(f(i)-g(j))\right\|_{X} \\
& \leqslant \frac{1}{n} \sum_{i=1}^{n} \sum_{j=1}^{n} a_{i j}\|f(i)-g(j)\|_{X} \leqslant\left(\frac{1}{n} \sum_{i=1}^{n} \sum_{j=1}^{n} a_{i j}\|f(i)-g(j)\|_{X}^{p}\right)^{1 / p} .
\end{aligned}
$$

Combining (157) and (158) we see that

$$
\left(\frac{1}{2 n} \sum_{i=1}^{n}\left(\left\|f_{0}(i)\right\|_{X}^{p}+\left\|g_{0}(i)\right\|_{X}^{p}\right)\right)^{1 / p} \leqslant \frac{2}{1-\lambda}\left(\frac{1}{n} \sum_{i=1}^{n} \sum_{j=1}^{n} a_{i j}\|f(i)-g(j)\|_{X}^{p}\right)^{1 / p} .
$$

But,

$$
\begin{gathered}
\left(\frac{1}{n^{2}} \sum_{i=1}^{n} \sum_{j=1}^{n}\|f(i)-g(j)\|_{X}^{p}\right)^{1 / p} \leqslant\|\bar{f}-\bar{g}\|_{X}+\left(\frac{1}{n^{2}} \sum_{i=1}^{n} \sum_{j=1}^{n}\left\|f_{0}(i)-g_{0}(j)\right\|_{X}^{p}\right)^{1 / p} \\
\leqslant\|\bar{f}-\bar{g}\|_{X}+\left(\frac{1}{n} \sum_{i=1}^{n} 2^{p-1}\left(\left\|f_{0}(i)\right\|_{X}^{p}+\left\|g_{0}(i)\right\|_{X}^{p}\right)\right)^{1 / p} \\
\underset{\leqslant}{\leqslant}\left(1+\frac{4}{1-\lambda}\right)\left(\frac{1}{n} \sum_{i=1}^{n} \sum_{j=1}^{n} a_{i j}\|f(i)-g(j)\|_{X}^{p}\right)^{1 / p},
\end{gathered}
$$

which implies the desired estimate (154).

6.1. Norm bounds need not imply nonlinear spectral gaps. One cannot bound $\gamma_{+}\left(A,\|\cdot\|_{X}^{p}\right)$ in terms of $\lambda_{X}^{(p)}(A)$ for a general Banach space $X$, as shown in the following example.

Lemma 6.2. For every $n \in \mathbb{N}$ there exists a $2^{n} \times 2^{n}$ symmetric stochastic matrix $A_{n}$ such that for every $p \in[1, \infty)$,

$$
\sup _{n \in \mathbb{N}} \gamma_{+}\left(A_{n},\|\cdot\|_{L_{1}}^{p}\right)<\infty
$$

yet

$$
\lim _{n \rightarrow \infty} \lambda_{L_{1}}^{(p)}\left(A_{n}\right)=1
$$

Proof. We use here the results and notation of Section 5. For every $t \in(0, \infty)$, the operator $e^{-t \Delta}$ is an averaging operator, since by (93) it corresponds to convolution with the Riesz kernel given in (94). Hence the $\mathbb{F}_{2}^{n} \times \mathbb{F}_{2}^{n}$ matrix $A_{n}$ whose entry at $(x, y) \in \mathbb{F}_{2}^{n} \times \mathbb{F}_{2}^{n}$ is

$$
\left(e^{-t \Delta} \delta_{x}\right)(y) \stackrel{(95)}{=}\left(\frac{1-e^{-t}}{2}\right)^{\|x-y\|_{1}}\left(\frac{1+e^{-t}}{2}\right)^{n-\|x-y\|_{1}}
$$

is symmetric and stochastic. Lemma 5.9 implies the validity of (161), so it remains to establish (160).

By Lemma 5.4 there exists $c_{p} \in(0, \infty)$ such that

$$
\lambda_{L_{2 p}}^{(2 p)}\left(A_{n}\right) \leqslant e^{-c_{p} \min \left\{t, t^{2}\right\}} .
$$


It therefore follows from Lemma 6.1 that

$$
\gamma_{+}\left(A_{n},\|\cdot\|_{L_{2 p}}^{2 p}\right) \leqslant\left(\frac{5-e^{-c_{p} \min \left\{t, t^{2}\right\}}}{1-e^{-c_{p} \min \left\{t, t^{2}\right\}}}\right)^{p} \stackrel{\text { def }}{=} C_{p}(t)<\infty .
$$

Since $L_{2}$ embeds isometrically into $L_{2 p}$ (see e.g. [67]), it follows that $\gamma_{+}\left(A_{n},\|\cdot\|_{L_{2}}^{2 p}\right) \leqslant C_{p}(t)$. It is a standard fact that $L_{1}$ equipped with the metric $d(f, g)=\sqrt{\|f-g\|_{1}}$ admits an isometric embedding into $L_{2}$ (for one of several possible simple proofs of this, see [48, Sec. 3]). It follows that $\gamma_{+}\left(A_{n},\|\cdot\|_{L_{1}}^{p}\right)=\gamma_{+}\left(A_{n}, d^{2 p}\right) \leqslant C_{p}(t)$.

6.2. A partial converse to Lemma 6.1 in uniformly convex spaces. Despite the validity of Lemma 6.2. Lemma 6.6 below is a partial converse to Lemma 6.1 that holds true if $X$ is uniformly convex. We start this section with a review of uniform convexity and smoothness; the material below will also be used in Section 6.3.

Let $\left(X,\|\cdot\|_{X}\right)$ be a normed space. The modulus of uniform convexity of $X$ is defined for $\varepsilon \in[0,2]$ as

$$
\delta_{X}(\varepsilon) \stackrel{\text { def }}{=} \inf \left\{1-\frac{\|x+y\|_{X}}{2}: x, y \in X,\|x\|_{X}=\|y\|_{X}=1,\|x-y\|_{X}=\varepsilon\right\} .
$$

$X$ is said to be uniformly convex if $\delta_{X}(\varepsilon)>0$ for all $\varepsilon \in(0,2]$. Furthermore, $X$ is said to have modulus of convexity of power type $p$ if there exists a constant $c \in(0, \infty)$ such that $\delta_{X}(\varepsilon) \geqslant c \varepsilon^{p}$ for all $\varepsilon \in[0,2]$. It is straightforward to check that in this case necessarily $p \geqslant 2$. By Proposition 7 in [5] (see also [14]), $X$ has modulus of convexity of power type $p$ if and only if there exists a constant $K \in[1, \infty)$ such that for every $x, y \in X$

$$
\|x\|_{X}^{p}+\frac{1}{K^{p}}\|y\|_{X}^{p} \leqslant \frac{\|x+y\|_{X}^{p}+\|x-y\|_{X}^{p}}{2} .
$$

The infimum over those $K$ for which (163) holds is called the $p$-convexity constant of $X$, and is denoted $K_{p}(X)$.

The modulus of uniform smoothness of $X$ is defined for $\tau \in(0, \infty)$ as

$$
\rho_{X}(\tau) \stackrel{\text { def }}{=} \sup \left\{\frac{\|x+\tau y\|_{X}+\|x-\tau y\|_{X}}{2}-1: x, y \in X,\|x\|_{X}=\|y\|_{X}=1\right\} \text {. }
$$

$X$ is said to be uniformly smooth if $\lim _{\tau \rightarrow 0} \rho_{X}(\tau) / \tau=0$. Furthermore, $X$ is said to have modulus of smoothness of power type $p$ if there exists a constant $C \in(0, \infty)$ such that $\rho_{X}(\tau) \leqslant C \tau^{p}$ for all $\tau \in(0, \infty)$. It is straightforward to check that in this case necessarily $p \in[1,2]$. It follows from [5] that $X$ has modulus of smoothness of power type $p$ if and only if there exists a constant $S \in[1, \infty)$ such that for every $x, y \in X$

$$
\frac{\|x+y\|_{X}^{p}+\|x-y\|_{X}^{p}}{2} \leqslant\|x\|_{X}^{p}+S^{p}\|y\|_{X}^{p} \text {. }
$$

The infimum over those $S$ for which (165) holds is called the $p$-smoothness constant of $X$, and is denoted $S_{p}(X)$.

The moduli appearing in (162) and (164) relate to each other via the following classical duality formula of Lindenstrauss [32].

$$
\rho_{X^{*}}(\tau)=\sup \left\{\frac{\tau \varepsilon}{2}-\delta_{X}(\varepsilon): \varepsilon \in[0,2]\right\} .
$$


Correspondingly, it was shown in [5, Lem. 5] that the best constants in (163) and (165) have the following duality relation.

$$
K_{p}(X)=S_{p /(p-1)}\left(X^{*}\right) .
$$

Observe that if $q \geqslant p$ then for all $x, y \in X$ we have

$$
\left(\frac{\|x+y\|_{X}^{p}+\|x-y\|_{X}^{p}}{2}\right)^{1 / p} \leqslant\left(\frac{\|x+y\|_{X}^{q}+\|x-y\|_{X}^{q}}{2}\right)^{1 / q}
$$

and

Hence,

$$
\left(\|x\|_{X}^{q}+\frac{1}{K^{q}}\|y\|_{X}^{q}\right)^{1 / q} \leqslant\left(\|x\|_{X}^{p}+\frac{1}{K^{p}}\|y\|_{X}^{p}\right)^{1 / p} .
$$

$$
q \geqslant p \Longrightarrow K_{q}(X) \leqslant K_{p}(X) .
$$

Similarly we have (though we will not use this fact later),

$$
q \leqslant p \Longrightarrow S_{q}(X) \leqslant S_{p}(X) \text {. }
$$

The following lemma can be deduced from a combination of results in [15, 14] and [5] (without the explicit dependence on $p, q$ ). A simple proof of the case $p=2$ of it is also contained in [50]; we include the natural adaptation of the argument to general $p \in(1,2]$ for the sake of completeness.

Lemma 6.3. For every $p \in(1,2], q \in[p, \infty)$, every Banach space $\left(X,\|\cdot\|_{X}\right.$ and every measure space $(\Omega, \mu)$, we have

$$
S_{p}\left(L_{q}(\mu, X)\right) \leqslant(5 p q)^{1 / p} S_{p}(X) .
$$

Proof. Fix $S>S_{p}(X)$. We will show that for every $x, y \in X$ we have

$$
\frac{\|x+y\|_{X}^{q}+\|x-y\|_{X}^{q}}{2} \leqslant\left(\|x\|_{X}^{p}+5 p q S^{p}\|y\|_{X}^{p}\right)^{q / p} .
$$

Assuming the validity of (168) for the moment, we complete the proof of Lemma 6.3 as follows. If $f, g \in L_{q}(\mu, X)$ then

$$
\begin{aligned}
\frac{\|f+g\|_{L_{q}(\mu, X)}^{p}+\|f-g\|_{L_{q}(\mu, X)}^{p}}{2} & \leqslant\left(\frac{\|f+g\|_{L_{q}(\mu, X)}^{q}+\|f-g\|_{L_{q}(\mu, X)}^{q}}{2}\right)^{p / q} \\
& =\left(\int_{\Omega} \frac{\|f+g\|_{X}^{q}+\|f-g\|_{X}^{q}}{2} d \mu\right)^{p / q} \\
& \leqslant\|\| f\left\|_{X}^{p}+5 p q S^{p}\right\| g\left\|_{X}^{p}\right\|_{L_{q / p}(\mu)} \\
& \leqslant\|f\|_{L_{q}(\mu, X)}^{p}+5 p q S^{p}\|g\|_{L_{q}(\mu, X)}^{p} .
\end{aligned}
$$

This proves that $S_{p}\left(L_{q}(\mu, X)\right)^{p} \leqslant 5 p q S_{p}(X)^{p}$, as desired.

It remains to prove (168). Since $\|y\|_{X}^{p}+5 p q S^{p}\|x\|_{X}^{p} \leqslant\|x\|_{X}^{p}+5 p q S^{p}\|y\|_{X}^{p}$ if $\|x\|_{X} \leqslant\|y\|_{X}$, it suffices to prove (168) under the additional assumption $\|y\|_{X} \leqslant\|x\|_{X}$. After normalization we may further assume that $\|x\|_{X}=1$ and $\|y\|_{X} \leqslant 1$.

Note that

$$
\left|\|x+y\|_{X}^{p}-\|x-y\|_{X}^{p}\right| \leqslant\left(1+\|y\|_{X}\right)^{p}-\left(1-\|y\|_{X}\right)^{p} \leqslant 2 p\|y\|_{X} .
$$


We claim that for every $\alpha \in[1, \infty)$ and $\beta \in[-1,1]$ we have

$$
\left(\frac{(1+\beta)^{\alpha}+(1-\beta)^{\alpha}}{2}\right)^{1 / \alpha} \leqslant 1+2 \alpha \beta^{2} .
$$

Indeed, by symmetry it suffices to prove (170) when $\beta \in[0,1]$. The left hand side of (170) is at most $\max \{1+\beta, 1-\beta\}=1+\beta$, which implies (170) when $\beta \geqslant 1 /(2 \alpha)$. We may therefore assume that $\beta \in[0,1 /(2 \alpha)]$, in which case the crude bound $(1+\beta)^{\alpha}+(1-\beta)^{\alpha} \leqslant 2+4 \alpha^{2} \beta^{2}$ follows from Taylor's expansion, implying (170) in this case as well.

Set

$$
b \stackrel{\text { def }}{=} \frac{\|x+y\|_{X}^{p}+\|x-y\|_{X}^{p}}{2} \text { and } \beta \stackrel{\text { def }}{=} \frac{\|x+y\|_{X}^{p}-\|x-y\|_{X}^{p}}{\|x+y\|_{X}^{p}+\|x-y\|_{X}^{p}},
$$

and define

$$
\theta \stackrel{\text { def }}{=}\left(\frac{(1+\beta)^{q / p}+(1-\beta)^{q / p}}{2}\right)^{p / q}-1 \in[0,1] .
$$

Observe that by convexity $b \geqslant 1$, and therefore

$$
\theta \stackrel{(170)}{\leqslant} 2 \frac{q}{p} \beta^{2} \stackrel{(169) \wedge(171)}{\leqslant} \frac{2 q}{p}\left(\frac{2 p\|y\|_{X}}{2 b}\right)^{2} \leqslant 2 p q\|y\|_{X}^{p},
$$

where we used the fact that $p \in[1,2]$ and $\|y\|_{X} \leqslant 1$. Now,

$$
\begin{aligned}
\frac{\|x+y\|_{X}^{q}+\|x-y\|_{X}^{q}}{2} \stackrel{(171)}{=} & \stackrel{(172)}{=}(b(1+\theta))^{q / p} \\
& \stackrel{(165)}{\leqslant}\left(\left(1+S^{p}\|y\|_{X}^{p}\right)(1+\theta)\right)^{q / p} \stackrel{(173)}{\leqslant}\left(1+5 p q S^{p}\|y\|_{X}^{p}\right)^{q / p} .
\end{aligned}
$$

By (166), Lemma 6.3 implies the following dual statement.

Corollary 6.4. For every $p \in[2, \infty), q \in(1, p]$, every Banach space $\left(X,\|\cdot\|_{X}\right)$ and every measure space $(\Omega, \mu)$, we have

$$
K_{p}\left(L_{q}(\mu, X)\right) \leqslant\left(\frac{5 p q}{(p-1)(q-1)}\right)^{1-1 / p} K_{p}(X) .
$$

The following lemma is stated and proved in [4] when $p=2$.

Lemma 6.5. Let $X$ be a normed space and $U$ a random vector in $X$ with $\mathbb{E}\left[\|U\|_{X}^{p}\right]<\infty$. Then

$$
\|\mathbb{E}[U]\|_{X}^{p}+\frac{1}{\left(2^{p-1}-1\right) K_{p}(X)^{p}} \mathbb{E}\left[\|U-\mathbb{E}[U]\|_{X}^{p}\right] \leqslant \mathbb{E}\left[\|U\|_{X}^{p}\right] .
$$

Proof. We repeat here the $p>2$ variant of the argument from [4] for the sake of completeness. Let $(\Omega, \operatorname{Pr})$ be the probability space on which $U$ is defined. Denote

$$
\theta \stackrel{\text { def }}{=} \inf \left\{\frac{\mathbb{E}\left[\|V\|_{X}^{p}\right]-\|\mathbb{E}[V]\|_{X}^{p}}{\mathbb{E}\left[\|V-\mathbb{E}[V]\|_{X}^{p}\right]}: V \in L_{p}(\Omega, X) \wedge \mathbb{E}\left[\|V-\mathbb{E}[V]\|_{X}^{p}\right]>0\right\} .
$$

Then $\theta \geqslant 0$. Our goal is to show that

$$
\theta \geqslant \frac{1}{\left(2^{p-1}-1\right) K_{p}(X)^{p}}
$$


Fix $\phi>\theta$. Then there exists a random vector $V_{0} \in L_{p}(\Omega, X)$ for which

$$
\phi \mathbb{E}\left[\left\|V_{0}-\mathbb{E}\left[V_{0}\right]\right\|_{X}^{p}\right]>\mathbb{E}\left[\left\|V_{0}\right\|_{X}^{p}\right]-\left\|\mathbb{E}\left[V_{0}\right]\right\|_{X}^{p} .
$$

Fix $K>K_{p}(X)$. Apply the inequality (163) to the vectors

$$
x=\frac{1}{2} V_{0}+\frac{1}{2} \mathbb{E}\left[V_{0}\right] \quad \text { and } \quad y=\frac{1}{2} V_{0}-\frac{1}{2} \mathbb{E}\left[V_{0}\right],
$$

to get the point-wise estimate

$$
2\left\|\frac{1}{2} V_{0}+\frac{1}{2} \mathbb{E}\left[V_{0}\right]\right\|_{X}^{p}+\frac{2}{K^{p}}\left\|\frac{1}{2} V_{0}-\frac{1}{2} \mathbb{E}\left[V_{0}\right]\right\|_{X}^{p} \leqslant\left\|V_{0}\right\|_{X}^{p}+\left\|\mathbb{E}\left[V_{0}\right]\right\|_{X}^{p} .
$$

Hence

$$
\begin{aligned}
& \phi \mathbb{E}\left[\left\|V_{0}-\mathbb{E}\left[V_{0}\right]\right\|_{X}^{p}\right] \stackrel{\sqrt{1766}}{>} \mathbb{E}\left[\left\|V_{0}\right\|_{X}^{p}\right]-\left\|\mathbb{E}\left[V_{0}\right]\right\|_{X}^{p} \\
& \stackrel{\sqrt[1177]{\geqslant}}{\geqslant} 2\left(\mathbb{E}\left[\left\|\frac{1}{2} V_{0}+\frac{1}{2} \mathbb{E}\left[V_{0}\right]\right\|_{X}^{p}\right]-\left\|\mathbb{E}\left[\frac{1}{2} V_{0}+\frac{1}{2} \mathbb{E}\left[V_{0}\right]\right]\right\|_{X}^{p}\right)+\frac{2}{K^{p}} \mathbb{E}\left[\left\|\frac{1}{2} V_{0}-\frac{1}{2} \mathbb{E}\left[V_{0}\right]\right\|_{X}^{p}\right] \\
& \stackrel{\sqrt[1744]{\geqslant}}{\geqslant} 2 \theta \mathbb{E}\left[\left\|\left(\frac{1}{2} V_{0}+\frac{1}{2} \mathbb{E}\left[V_{0}\right]\right)-\mathbb{E}\left[\frac{1}{2} V_{0}+\frac{1}{2} \mathbb{E}\left[V_{0}\right]\right]\right\|_{X}^{p}\right]+\frac{2}{K^{p}} \mathbb{E}\left[\left\|\frac{1}{2} V_{0}-\frac{1}{2} \mathbb{E}\left[V_{0}\right]\right\|_{X}^{p}\right] \\
& =\left(\frac{\theta}{2^{p-1}}+\frac{1}{2^{p-1} K^{p}}\right) \mathbb{E}\left[\left\|V_{0}-\mathbb{E}\left[V_{0}\right]\right\|_{X}^{p}\right] .
\end{aligned}
$$

Thus

$$
\phi \geqslant \frac{\theta}{2^{p-1}}+\frac{1}{2^{p-1} K^{p}} .
$$

Since (178) holds for all $\phi>\theta$ and $K>K_{p}(X)$, the desired lower bound (175) follows.

Lemma 6.6. Fix $p \in[2, \infty)$ and let $X$ be a normed space with $K_{p}(X)<\infty$. Then for every $n \times n$ symmetric stochastic matrix $A=\left(a_{i j}\right)$ we have

$$
\lambda_{X}^{(p)}(A) \leqslant\left(1-\frac{1}{\left(2^{p-1}-1\right) K_{p}(X)^{p} \gamma_{+}\left(A,\|\cdot\|_{X}^{p}\right)}\right)^{1 / p} .
$$

Proof. Fix $\gamma_{+}>\gamma_{+}\left(A,\|\cdot\|_{X}^{p}\right)$ and $f \in L_{p}^{n}(X)_{0}$. For every $i \in\{1, \ldots, n\}$ consider the random vector $U_{i} \in X$ given by

$$
\operatorname{Pr}\left[U_{i}=f(j)\right]=a_{i j} .
$$

Lemma 6.5 implies that

$$
\left\|\sum_{j=1}^{n} a_{i j} f(j)\right\|_{X}^{p} \leqslant \sum_{j=1}^{n} a_{i j}\|f(j)\|_{X}^{p}-\frac{1}{\left(2^{p-1}-1\right) K_{p}(X)^{p}} \sum_{j=1}^{n} a_{i j}\left\|f(j)-\sum_{k=1}^{n} a_{i k} f(k)\right\|_{X}^{p} .
$$

Define for $i \in\{1, \ldots, n\}$,

$$
g(i)=\mathbb{E}\left[U_{i}\right]=\sum_{k=1}^{n} a_{i k} f(k) .
$$


By averaging (179) over $i \in\{1, \ldots, n\}$ we see that

$$
\begin{aligned}
\left\|\left(A \otimes I_{X}^{n}\right) f\right\|_{L_{p}^{n}(X)}^{p} & =\frac{1}{n} \sum_{i=1}^{n}\left\|\sum_{j=1}^{n} a_{i j} f(j)\right\|_{X}^{p} \\
& \leqslant \frac{1}{n} \sum_{i=1}^{n} \sum_{j=1}^{n} a_{i j}\|f(j)\|_{X}^{p}-\frac{1}{n\left(2^{p-1}-1\right) K_{p}(X)^{p}} \sum_{i=1}^{n} \sum_{j=1}^{n} a_{i j}\|f(j)-g(i)\|_{X}^{p} \\
& =\|f\|_{L_{p}^{n}(X)}^{p}-\frac{1}{n\left(2^{p-1}-1\right) K_{p}(X)^{p}} \sum_{i=1}^{n} \sum_{j=1}^{n} a_{i j}\|f(j)-g(i)\|_{X}^{p} . \quad(180)
\end{aligned}
$$

The definition of $\gamma_{+}\left(A,\|\cdot\|_{X}^{p}\right)$ implies that

$$
\begin{aligned}
\frac{1}{n} \sum_{i=1}^{n} \sum_{j=1}^{n} a_{i j}\|f(j)-g(i)\|_{X}^{p} \geqslant \frac{1}{\gamma_{+} n^{2}} \sum_{i=1}^{n} \sum_{j=1}^{n}\|f(j)-g(i)\|_{X}^{p} \\
\geqslant \frac{1}{\gamma_{+} n} \sum_{j=1}^{n}\left\|f(j)-\frac{1}{n} \sum_{i=1}^{n} g(i)\right\|_{X}^{p}=\frac{1}{\gamma_{+} n} \sum_{j=1}^{n}\|f(j)\|_{X}^{p}=\frac{1}{\gamma_{+}}\|f\|_{L_{p}^{n}(X)}^{p}
\end{aligned}
$$

where we used the fact that since $f \in L_{p}^{n}(X)_{0}$ we have

$$
\sum_{i=1}^{n} g(i)=\sum_{k=1}^{n}\left(\sum_{i=1}^{n} a_{i k}\right) f(k)=\sum_{k=1}^{n} f(k)=0 .
$$

Substituting (181) into (180) yields the bound

$$
\left\|\left(A \otimes I_{X}^{n}\right) f\right\|_{L_{p}^{n}(X)}^{p} \leqslant\left(1-\frac{1}{\left(2^{p-1}-1\right) K_{p}(X)^{p} \gamma_{+}}\right)\|f\|_{L_{p}^{n}(X)}^{p} .
$$

Since (182) holds for every $f \in L_{p}^{n}(X)_{0}$ and $\gamma_{+}>\gamma_{+}\left(A,\|\cdot\|_{X}^{p}\right)$, inequality (182) implies the required bound on $\lambda_{X}^{(p)}(A)=\left\|A \otimes I_{X}^{n}\right\|_{L_{p}^{n}(X)_{0} \rightarrow L_{p}^{n}(X)_{0}}$.

Theorem 6.7. Fix $p \in[2, \infty)$ and $t \in \mathbb{N}$. Let $X$ be a normed space with $K_{p}(X)<\infty$. Then for every $n \times n$ symmetric stochastic matrix $A=\left(a_{i j}\right)$ we have

$$
\gamma_{+}\left(A^{t},\|\cdot\|_{X}^{p}\right) \leqslant\left[4 K_{p}(X)\right]^{p^{2}} \cdot \max \left\{1,\left(\frac{\gamma_{+}\left(A,\|\cdot\|_{X}^{p}\right)}{t}\right)^{p}\right\} .
$$

Proof. Note that since $A \otimes I_{X}^{n}$ preserves $L_{p}^{n}(X)_{0}$ we have

$$
\begin{aligned}
\lambda_{X}^{(p)}\left(A^{t}\right)=\left\|A^{t} \otimes I_{X}^{n}\right\|_{L_{p}^{n}(X)_{0} \rightarrow L_{p}^{n}(X)_{0}}=\left\|\left(A \otimes I_{X}^{n}\right)^{t}\right\|_{L_{p}^{n}(X)_{0} \rightarrow L_{p}^{n}(X)_{0}} & \\
& \leqslant\left\|A \otimes I_{X}^{n}\right\|_{L_{p}^{n}(X)_{0} \rightarrow L_{p}^{n}(X)_{0}}^{t}=\lambda_{X}^{(p)}(A)^{t} .
\end{aligned}
$$

Lemma 6.1 applied to the matrix $A^{t}$, in combination with (183), yields the bound

$$
\gamma_{+}\left(A^{t},\|\cdot\|_{X}^{p}\right) \leqslant\left(\frac{5-\lambda_{X}^{(p)}(A)^{t}}{1-\lambda_{X}^{(p)}(A)^{t}}\right)^{p} \leqslant\left(\frac{5}{1-\lambda_{X}^{(p)}(A)^{t}}\right)^{p} .
$$


On the other hand, using Lemma 6.6 we have

$$
\begin{aligned}
& \lambda_{X}^{(p)}(A) \leqslant\left(1-\frac{1}{\left(2^{p-1}-1\right) K_{p}(X)^{p} \gamma_{+}\left(A,\|\cdot\|_{X}^{p}\right)}\right)^{1 / p} \\
& \quad \leqslant \exp \left(-\frac{1}{p\left(2^{p-1}-1\right) K_{p}(X)^{p} \gamma_{+}\left(A,\|\cdot\|_{X}^{p}\right)}\right) .
\end{aligned}
$$

Thus

$$
\begin{aligned}
1-\lambda_{X}^{(p)}(A)^{t} \stackrel{(185)}{\geqslant} 1-\exp \left(-\frac{t}{p\left(2^{p-1}-1\right) K_{p}(X)^{p} \gamma_{+}\left(A,\|\cdot\|_{X}^{p}\right)}\right) \\
\geqslant \frac{1}{2} \min \left\{1, \frac{t}{p\left(2^{p-1}-1\right) K_{p}(X)^{p} \gamma_{+}\left(A,\|\cdot\|_{X}^{p}\right)}\right\} .
\end{aligned}
$$

The required result is now a combination of (186) and (184).

6.3. Martingale inequalities and metric Markov cotype. Let $X$ be a Banach space with $K_{p}(X)<\infty$. Assume that $\left\{M_{k}\right\}_{k=0}^{n} \subseteq X$ is a martingale with respect to the filtration $\mathcal{F}_{0} \subseteq \mathcal{F}_{1} \subseteq \cdots \subseteq \mathcal{F}_{n-1}$, i.e., $\mathbb{E}\left[M_{i+1} \mid \mathcal{F}_{i}\right]=M_{i}$ for every $i \in\{0,1, \ldots, n-1\}$. Lemma 6.5 implies that

$$
\begin{gathered}
\mathbb{E}\left[\left\|M_{n}-M_{0}\right\|_{X}^{p} \mid \mathcal{F}_{n-1}\right] \geqslant\left\|\mathbb{E}\left[M_{n}-M_{0} \mid \mathcal{F}_{n-1}\right]\right\|_{X}^{p} \\
\quad+\frac{1}{\left(2^{p-1}-1\right) K_{p}(X)^{p}} \mathbb{E}\left[\left\|M_{n}-M_{0}-\mathbb{E}\left[M_{n}-M_{0} \mid \mathcal{F}_{n-1}\right]\right\|_{X}^{p} \mid \mathcal{F}_{n-1}\right] \\
=\left\|M_{n-1}-M_{0}\right\|_{X}^{p}+\frac{1}{\left(2^{p-1}-1\right) K_{p}(X)^{p}} \mathbb{E}\left[\left\|M_{n}-M_{n-1}\right\|_{X}^{p} \mid \mathcal{F}_{n-1}\right] .
\end{gathered}
$$

Taking expectation in (187) yields the estimate

$$
\mathbb{E}\left[\left\|M_{n}-M_{0}\right\|_{X}^{p}\right] \geqslant \mathbb{E}\left[\left\|M_{n-1}-M_{0}\right\|_{X}^{p}\right]+\frac{1}{\left(2^{p-1}-1\right) K_{p}(X)^{p}} \mathbb{E}\left[\left\|M_{n}-M_{n-1}\right\|_{X}^{p}\right] .
$$

Iterating this argument we obtain the following famous inequality of Pisier [56], which will be used crucially in what follows.

Theorem 6.8 (Pisier's martingale inequality). Let $X$ be a Banach space with $K_{p}(X)<\infty$. Suppose that $\left\{M_{k}\right\}_{k=0}^{n} \subseteq X$ is a martingale (with respect some filtration). Then

$$
\mathbb{E}\left[\left\|M_{n}-M_{0}\right\|_{X}^{p}\right] \geqslant \frac{1}{\left(2^{p-1}-1\right) K_{p}(X)^{p}} \sum_{k=1}^{n} \mathbb{E}\left[\left\|M_{k}-M_{k-1}\right\|_{X}^{p}\right] .
$$

We also need the following variant of Pisier's inequality.

Corollary 6.9. Fix $p \in[2, \infty), q \in(1, \infty)$ and let $X$ be a normed space with $K_{p}(X)<\infty$. Then for every q-integrable martingale $\left\{M_{k}\right\}_{k=0}^{n} \subseteq X$, if $q \in[p, \infty)$ then

$$
\mathbb{E}\left[\left\|M_{n}-M_{0}\right\|_{X}^{q}\right] \geqslant \frac{1}{\left(2^{q-1}-1\right) K_{p}(X)^{q}} \sum_{k=1}^{n} \mathbb{E}\left[\left\|M_{k}-M_{k-1}\right\|_{X}^{q}\right] .
$$


and if $q \in(1, p]$, then

$$
\mathbb{E}\left[\left\|M_{n}-M_{0}\right\|_{X}^{q}\right] \geqslant \frac{((1-1 / p)(1-1 / q))^{q(1-1 / p)}}{5^{q(1-1 / p)}\left(2 K_{p}(X)\right)^{q} n^{1-q / p}} \sum_{k=1}^{n} \mathbb{E}\left[\left\|M_{k}-M_{k-1}\right\|_{X}^{q}\right] .
$$

Proof. Denote the probability space on which the martingale $\left\{M_{k}\right\}_{k=0}^{n}$ is defined by $(\Omega, \mu)$. Suppose also that $\mathcal{F}_{0} \subseteq \mathcal{F}_{1} \subseteq \cdots \subseteq \mathcal{F}_{n-1}$ is the filtration with respect to which $\left\{M_{k}\right\}_{k=0}^{n}$ is a martingale.

If $p \leqslant q$ then (188) is an immediate consequence of Theorem 6.8 and (167). If $q \in(1, p]$ then by Corollary 6.4 we have

$$
K \stackrel{\text { def }}{=} K_{p}\left(L_{q}(\mu, X)\right) \leqslant\left(\frac{5 p q}{(p-1)(q-1)}\right)^{1-1 / p} K_{p}(X)
$$

We can therefore apply (163) to the following two vectors in $L_{q}(\mu, X)$.

$$
x=M_{n-1}-M_{0}+\frac{M_{n}-M_{n-1}}{2} \text { and } y=\frac{M_{n}-M_{n-1}}{2},
$$

yielding the following estimate.

$$
\begin{aligned}
\left(\mathbb{E}\left[\left\|M_{n-1}-M_{0}+\frac{M_{n}-M_{n-1}}{2}\right\|_{X}^{q}\right]\right)^{p / q}+\frac{1}{(2 K)^{p}}\left(\mathbb{E}\left[\left\|M_{n}-M_{n-1}\right\|_{X}^{q}\right]\right)^{p / q} \\
\leqslant \frac{\left(\mathbb{E}\left[\left\|M_{n}-M_{0}\right\|_{X}^{q}\right]\right)^{p / q}+\left(\mathbb{E}\left[\left\|M_{n-1}-M_{0}\right\|_{X}^{q}\right]\right)^{p / q}}{2} .
\end{aligned}
$$

Now,

$$
\mathbb{E}\left[\left\|M_{n-1}-M_{0}\right\|_{X}^{q}\right]=\mathbb{E}\left[\left\|M_{n-1}-M_{0}+\mathbb{E}\left[M_{n}-M_{n-1} \mid \mathcal{F}_{n-1}\right]\right\|_{X}^{q}\right] \leqslant \mathbb{E}\left[\left\|M_{n}-M_{0}\right\|_{X}^{q}\right],
$$

and

$$
\begin{aligned}
\mathbb{E}\left[\left\|M_{n-1}-M_{0}\right\|_{X}^{q}\right]=\mathbb{E}\left[\left\|M_{n-1}-M_{0}+\mathbb{E}\left[\frac{M_{n}-M_{n-1}}{2} \mid \mathcal{F}_{n-1}\right]\right\|_{X}^{q}\right] \\
\leqslant \mathbb{E}\left[\left\|M_{n-1}-M_{0}+\frac{M_{n}-M_{n-1}}{2}\right\|_{X}^{q}\right] .
\end{aligned}
$$

Thus (190) implies that

$$
\left(\mathbb{E}\left[\left\|M_{n-1}-M_{0}\right\|_{X}^{q}\right]\right)^{p / q}+\frac{1}{(2 K)^{p}}\left(\mathbb{E}\left[\left\|M_{n}-M_{n-1}\right\|_{X}^{q}\right]\right)^{p / q} \leqslant\left(\mathbb{E}\left[\left\|M_{n}-M_{0}\right\|_{X}^{q}\right]\right)^{p / q} .
$$

Applying (191) inductively we get the lower bound

$$
\begin{aligned}
(2 K)^{p}\left(\mathbb{E}\left[\left\|M_{n}-M_{0}\right\|_{X}^{q}\right]\right)^{p / q} \geqslant \sum_{k=1}^{n}\left(\mathbb{E}\left[\left\|M_{k}-M_{k-1}\right\|_{X}^{q}\right]\right)^{p / q} & \\
& \geqslant \frac{1}{n^{\frac{p}{q}-1}}\left(\sum_{k=1}^{n} \mathbb{E}\left[\left\|M_{k}-M_{k-1}\right\|_{X}^{q}\right]\right)^{p / q},
\end{aligned}
$$

which is precisely (189). 
We are now in position to prove the main theorem of this section, which establishes metric Markov cotype $p$ inequalities (recall Definition 1.4) for Banach space with modulus of convexity of power type $p$. An important theorem of Pisier [56] asserts that if a normed space $\left(X,\|\cdot\|_{X}\right)$ is super-reflexive then there exists $p \in[2, \infty)$ such that $K_{p}(X)<\infty$. Thus the case $q=2$ of Theorem 6.10 below corresponds to Theorem 1.8 .

Theorem 6.10. Fix $p \in[2, \infty)$ and let $\left(X,\|\cdot\|_{X}\right)$ be a normed space with $K_{p}(X)<\infty$. Then for every $m, n \in \mathbb{N}$, every $n \times n$ symmetric stochastic matrix $A=\left(a_{i j}\right)$ and every $x_{1}, \ldots, x_{n} \in X$ there exist $y_{1}, \ldots y_{n} \in X$ such that for all $q \in(1, \infty)$,

$$
\begin{aligned}
& \max \left\{\sum_{i=1}^{n}\left\|x_{i}-y_{i}\right\|_{X}^{q},\left(\frac{((1-1 / p)(1-1 / q))^{1-1 / p}}{32 \cdot 5^{1-1 / p} K_{p}(X)}\right)^{q} m^{\min \{1, q / p\}} \sum_{i=1}^{n} \sum_{j=1}^{n} a_{i j}\left\|y_{i}-y_{j}\right\|_{X}^{q}\right\} \\
& \leqslant \sum_{i=1}^{n} \sum_{j=1}^{n} \mathscr{A}_{m}(A)_{i j}\left\|x_{i}-x_{j}\right\|_{X}^{q}
\end{aligned}
$$

In particular, for $q=2$ we have

$$
\sum_{i=1}^{n}\left\|x_{i}-y_{i}\right\|_{X}^{2}+m^{2 / p} \sum_{i=1}^{n} \sum_{j=1}^{n} a_{i j}\left\|y_{i}-y_{j}\right\|_{X}^{2} \leqslant\left(32 K_{p}(X)\right)^{2} \sum_{i=1}^{n} \sum_{j=1}^{n} \mathscr{A}_{m}(A)_{i j}\left\|x_{i}-x_{j}\right\|_{X}^{2},
$$

Thus $X$ has metric Markov cotype $p$ with exponent 2 and with $C_{p}^{(2)}(X) \leqslant 32 K_{p}(X)$.

Proof. Define $f \in L_{p}^{n}(X)$ by $f(i)=x_{i}$. For every $\ell \in\{1, \ldots, n\}$ let

$$
Z_{0}^{(\ell)}, Z_{1}^{(\ell)}, Z_{2}^{(\ell)}, \ldots
$$

be the Markov chain on $\{1, \ldots, n\}$ which starts at $\ell$ and has transition matrix $A$. In other words $Z_{0}^{(\ell)}=\ell$ with probability one and for all $t \in\{1, \ldots, m\}$ and $i, j \in\{1, \ldots, n\}$ we have

$$
\operatorname{Pr}\left[Z_{t}^{(\ell)}=j \mid Z_{t-1}^{(\ell)}=i\right]=a_{i j}
$$

For $t \in\{0, \ldots, m\}$ define $f_{t} \in L_{p}^{n}(X)$ by

$$
f_{t} \stackrel{\text { def }}{=}\left(A^{m-t} \otimes I_{X}^{n}\right) f .
$$

Observe that if we set

$$
M_{t}^{(\ell)} \stackrel{\text { def }}{=} f_{t}\left(Z_{t}^{(\ell)}\right)
$$

then $M_{0}^{(\ell)}, M_{1}^{(\ell)}, \ldots, M_{m}^{(\ell)}$ is a martingale with respect to the filtration induced by the random variables $Z_{0}^{(\ell)}, Z_{1}^{(\ell)}, \ldots, Z_{m}^{(\ell)}$. Indeed, writing $L=A \otimes I_{X}^{n}$ we have for every $t \geqslant 1$,

$$
\begin{aligned}
\mathbb{E}\left[M_{t}^{(\ell)} \mid Z_{0}^{(\ell)}, \ldots, Z_{t-1}^{(\ell)}\right]=\mathbb{E}\left[\left(L^{m-t} f\right)\left(Z_{t}^{(\ell)}\right) \mid Z_{t-1}^{(\ell)}\right]=L^{m-t} \mathbb{E}\left[f\left(Z_{t}^{(\ell)}\right) \mid Z_{t-1}^{(\ell)}\right] \\
=L^{m-t}(L f)\left(Z_{t-1}^{(\ell)}\right)=\left(L^{m-(t-1)} f\right)\left(Z_{t-1}^{(\ell)}\right)=M_{t-1}^{(\ell)} .
\end{aligned}
$$

Write

$$
K \stackrel{\text { def }}{=} \begin{cases}\left(2^{q-1}-1\right) K_{p}(X)^{q} & \text { if } q \in[p, \infty) \\ \frac{5^{q(1-1 / p)}\left(2 K_{p}(X)\right)^{q} m^{1-q / p}}{((1-1 / p)(1-1 / q))^{q(1-1 / p)}} & \text { if } q \in(1, p)\end{cases}
$$


Then Corollary 6.9 applied to the martingale $\left\{M_{t}^{(\ell)}\right\}_{t=0}^{m}$ implies that

$$
K \mathbb{E}\left[\left\|f\left(Z_{m}^{(\ell)}\right)-\left(L^{m} f\right)(\ell)\right\|_{X}^{q}\right] \geqslant \sum_{t=1}^{m} \mathbb{E}\left[\left\|\left(L^{m-t} f\right)\left(Z_{t}^{(\ell)}\right)-\left(L^{m-t+1} f\right)\left(Z_{t-1}^{(\ell)}\right)\right\|_{X}^{q}\right] .
$$

Let $\left\{Z_{t}\right\}_{t=0}^{\infty}$ be the Markov chain with transition matrix $A$ such that $Z_{0}$ is uniformly distributed on $\{1, \ldots, n\}$. Averaging (194) over $\ell \in\{1, \ldots, n\}$ yields the inequality

$$
K \mathbb{E}\left[\left\|f\left(Z_{m}\right)-\left(L^{m} f\right)\left(Z_{0}\right)\right\|_{X}^{q}\right] \geqslant \sum_{t=1}^{m} \mathbb{E}\left[\left\|\left(L^{m-t} f\right)\left(Z_{t}\right)-\left(L^{m-t+1} f\right)\left(Z_{t-1}\right)\right\|_{X}^{q}\right],
$$

Which is the same as

$$
\begin{aligned}
K \sum_{i=1}^{n} \sum_{j=1}^{n}\left(A^{m}\right)_{i j}\left\|f(i)-\left(L^{m} f\right)(j)\right\|_{X}^{q} & \\
& \geqslant \sum_{t=1}^{m} \sum_{i=1}^{n} \sum_{j=1}^{n} a_{i j}\left\|\left(L^{m-t} f\right)(i)-\left(L^{m-t+1} f\right)(j)\right\|_{X}^{q} .
\end{aligned}
$$

In order to bound the right-hand side of (196), for every $i \in\{1 \ldots, n\}$ consider the vector

$$
y_{i} \stackrel{\text { def }}{=} \frac{1}{m} \sum_{j=1}^{n} \sum_{s=0}^{m-1}\left(A^{s}\right)_{i j} x_{j}=\frac{1}{m} \sum_{s=0}^{m-1} L^{s} f(i)
$$

and observe that

$$
\frac{1}{m} \sum_{s=1}^{m} L^{s} f(i)=y_{i}-\frac{1}{m} x_{i}+\frac{1}{m} L^{m} f(i)=y_{i}-\frac{1}{m} \sum_{r=1}^{n}\left(A^{m}\right)_{i r}\left(x_{i}-x_{r}\right) .
$$

Therefore, using convexity we have:

$$
\begin{aligned}
& \sum_{t=1}^{m} \sum_{i=1}^{n} \sum_{j=1}^{n} a_{i j}\left\|\left(L^{m-t} f\right)(i)-\left(L^{m-t+1} f\right)(j)\right\|_{X}^{q} \\
& \geqslant m \sum_{i=1}^{n} \sum_{j=1}^{n} a_{i j}\left\|\frac{1}{m} \sum_{t=1}^{m}\left(\left(L^{m-t} f\right)(i)-\left(L^{m-t+1} f\right)(j)\right)\right\|_{X}^{q} \\
& \stackrel{m}{=} \sum_{i=1}^{n} \sum_{j=1}^{n} a_{i j}\left\|y_{i}-y_{j}+\frac{1}{m} \sum_{r=1}^{n}\left(A^{m}\right)_{j r}\left(x_{j}-x_{r}\right)\right\|_{X}^{q} \\
& \geqslant \quad \frac{m}{2^{q-1}} \sum_{i=1}^{n} \sum_{j=1}^{n} a_{i j}\left\|y_{i}-y_{j}\right\|_{X}^{q}-\frac{1}{m^{q-1}} \sum_{i=1}^{n} \sum_{j=1}^{n} a_{i j}\left\|\sum_{r=1}^{n}\left(A^{m}\right)_{j r}\left(x_{j}-x_{r}\right)\right\|_{X}^{q} \\
& =\frac{m}{2^{q-1}} \sum_{i=1}^{n} \sum_{j=1}^{n} a_{i j}\left\|y_{i}-y_{j}\right\|_{X}^{q}-\frac{1}{m^{q-1}} \sum_{j=1}^{n}\left\|\sum_{r=1}^{n}\left(A^{m}\right)_{j r}\left(x_{j}-x_{r}\right)\right\|_{X}^{q} \\
& \geqslant \quad \frac{m}{2^{q-1}} \sum_{i=1}^{n} \sum_{j=1}^{n} a_{i j}\left\|y_{i}-y_{j}\right\|_{X}^{q}-\frac{1}{m^{q-1}} \sum_{j=1}^{n} \sum_{r=1}^{n}\left(A^{m}\right)_{j r}\left\|x_{j}-x_{r}\right\|_{X}^{q} .
\end{aligned}
$$


At the same time, we can bound the left-hand side of (196) as follows:

$$
\begin{aligned}
& \sum_{i=1}^{n} \sum_{j=1}^{n}\left(A^{m}\right)_{i j}\left\|f(i)-\left(L^{m} f\right)(j)\right\|_{X}^{q}=\sum_{i=1}^{n} \sum_{j=1}^{n}\left(A^{m}\right)_{i j}\left\|x_{i}-\sum_{r=1}^{n}\left(A^{m}\right)_{j r} x_{r}\right\|_{X}^{q} \\
& \leqslant \sum_{i=1}^{n} \sum_{j=1}^{n} \sum_{r=1}^{n}\left(A^{m}\right)_{i j}\left(A^{m}\right)_{j r}\left\|x_{i}-x_{r}\right\|_{X}^{q} \\
& \leqslant 2^{q-1} \sum_{i=1}^{n} \sum_{j=1}^{n} \sum_{r=1}^{n}\left(A^{m}\right)_{i j}\left(A^{m}\right)_{j r}\left(\left\|x_{i}-x_{j}\right\|_{X}^{q}+\left\|x_{j}-x_{r}\right\|_{X}^{q}\right) \\
& =2^{q} \sum_{i=1}^{n} \sum_{j=1}^{n}\left(A^{m}\right)_{i j}\left\|x_{i}-x_{j}\right\|_{X}^{q} .
\end{aligned}
$$

We note that,

$$
\begin{aligned}
& \sum_{i=1}^{n} \sum_{j=1}^{n}\left(A^{m}\right)_{i j}\left\|x_{i}-x_{j}\right\|_{X}^{q}=\sum_{i=1}^{n} \sum_{j=1}^{n}\left(\frac{1}{m} \sum_{t=0}^{m-1} A^{t} A^{m-t}\right)_{i j}\left\|x_{i}-x_{j}\right\|_{X}^{q} \\
& \quad \leqslant \frac{2^{q-1}}{m} \sum_{i=1}^{n} \sum_{j=1}^{n} \sum_{r=1}^{n} \sum_{t=0}^{m-1}\left(A^{t}\right)_{i r}\left(A^{m-t}\right)_{r j}\left(\left\|x_{i}-x_{r}\right\|_{X}^{q}+\left\|x_{r}-x_{j}\right\|_{X}^{q}\right) \\
& \quad=2^{q-1} \sum_{i=1}^{n} \sum_{r=1}^{n}\left(\frac{1}{m} \sum_{t=0}^{m-1} A^{t}\right)_{i r}\left\|x_{i}-x_{r}\right\|_{X}^{q}+2^{q-1} \sum_{j=1}^{n} \sum_{r=1}^{n}\left(\frac{1}{m} \sum_{t=0}^{m-1} A^{m-t}\right)_{r j}\left\|x_{r}-x_{j}\right\|_{X}^{q} \\
& \quad=2^{q} \sum_{i=1}^{n} \sum_{j=1}^{n} \mathscr{A}_{m}(A)_{i j}\left\|x_{i}-x_{j}\right\|_{X}^{q}+\frac{2^{q-1}}{m} \sum_{i=1}^{n} \sum_{j=1}^{n}\left(A^{m}\right)_{i j}\left\|x_{i}-x_{j}\right\|_{X}^{q},
\end{aligned}
$$

which, assuming that $m \geqslant 2^{q}$ gives the following bound.

$$
\sum_{i=1}^{n} \sum_{j=1}^{n}\left(A^{m}\right)_{i j}\left\|x_{i}-x_{j}\right\|_{X}^{q} \leqslant 2^{q+1} \sum_{i=1}^{n} \sum_{j=1}^{n} \mathscr{A}_{m}(A)_{i j}\left\|x_{i}-x_{j}\right\|_{X}^{q} .
$$

On the other hand, if $m \leqslant 2^{q}$ then

$$
\begin{aligned}
& \sum_{i=1}^{n} \sum_{j=1}^{n}\left(A^{m}\right)_{i j}\left\|x_{i}-x_{j}\right\|_{X}^{q} \leqslant \sum_{i=1}^{n} \sum_{j=1}^{n} \sum_{r=1}^{n} a_{i r}\left(A^{m-1}\right)_{r j}\left\|x_{i}-x_{j}\right\|_{X}^{q} \\
& \quad \leqslant 2^{q-1} \sum_{i=1}^{n} \sum_{j=1}^{n} \sum_{r=1}^{n} a_{i r}\left(A^{m-1}\right)_{r j}\left(\left\|x_{i}-x_{r}\right\|_{X}^{q}+\left\|x_{r}-x_{j}\right\|_{X}^{q}\right) \\
& \quad=2^{q-1} \sum_{i=1}^{n} \sum_{j=1}^{n} a_{i j}\left\|x_{i}-x_{j}\right\|_{X}^{q}+2^{q-1} \sum_{i=1}^{n} \sum_{j=1}^{n}\left(A^{m-1}\right)_{i j}\left\|x_{i}-x_{j}\right\|_{X}^{q} \\
& \leqslant 2^{q-1} m \sum_{i=1}^{n} \sum_{j=1}^{n} \mathscr{A}_{m}(A)_{i j}\left\|x_{i}-x_{j}\right\|_{X}^{q} \leqslant 2^{2 q-1} \sum_{i=1}^{n} \sum_{j=1}^{n} \mathscr{A}_{m}(A)_{i j}\left\|x_{i}-x_{j}\right\|_{X}^{q} .
\end{aligned}
$$


Thus, by combining (201) and (202) we get the estimate

$$
\sum_{i=1}^{n} \sum_{j=1}^{n}\left(A^{m}\right)_{i j}\left\|x_{i}-x_{j}\right\|_{X}^{q} \leqslant 4^{q} \sum_{i=1}^{n} \sum_{j=1}^{n} \mathscr{A}_{m}(A)_{i j}\left\|x_{i}-x_{j}\right\|_{X}^{q}
$$

Substituting (199) and (200) into (196) yields the bound

$$
\begin{aligned}
m \sum_{i=1}^{n} \sum_{j=1}^{n} a_{i j}\left\|y_{i}-y_{j}\right\|_{X}^{q} \leqslant 4^{q} K \sum_{i=1}^{n} \sum_{j=1}^{n}\left(A^{m}\right)_{i j}\left\|x_{i}-x_{j}\right\|_{X}^{q} & \stackrel{(\sqrt{203})}{\leqslant} 2^{4 q} K \sum_{i=1}^{n} \sum_{j=1}^{n} \mathscr{A}_{m}(A)_{i j}\left\|x_{i}-x_{j}\right\|_{X}^{q} .
\end{aligned}
$$

At the same time,

$$
\sum_{i=1}^{n}\left\|x_{i}-y_{i}\right\|_{X}^{q}=\sum_{i=1}^{n}\left\|\frac{1}{m} \sum_{j=1}^{n} \sum_{t=0}^{m-1}\left(A^{t}\right)_{i j}\left(x_{i}-x_{j}\right)\right\|_{X}^{q} \leqslant \sum_{i=1}^{n} \sum_{j=1}^{n} \mathscr{A}_{m}(A)_{i j}\left\|x_{i}-x_{j}\right\|_{X}^{q} .
$$

Recalling (193), the desired inequality (192) is now a combination of (204) and (205).

\section{Construction of the Base Graph}

For $t \in(0, \infty)$ and $n \in \mathbb{N}$ write

$$
\tau_{t} \stackrel{\text { def }}{=} \frac{1-e^{-t}}{2} \text { and } \quad \sigma_{t}^{n} \stackrel{\text { def }}{=} \tau_{t}^{4 \tau_{t} n}\left(1-\tau_{t}\right)^{\left(1-4 \tau_{t}\right) n} .
$$

We also define $e_{t}^{n}:\{0, \ldots, n\} \rightarrow \mathbb{N} \cup\{0\}$ by

$$
e_{t}^{n}(k) \stackrel{\text { def }}{=}\left\lfloor\frac{\tau_{t}^{k}\left(1-\tau_{t}\right)^{n-k}}{\sigma_{t}^{n}}\right\rfloor .
$$

The following lemma records elementary estimates on binomial sums that will be useful for us later.

Lemma 7.1. Fix $t \in(0,1 / 4)$ and $n \in \mathbb{N} \cap[8000, \infty)$ such that

$$
\tau_{t} \geqslant \frac{1}{3 \sqrt{n}}
$$

Then

$$
\frac{1}{3 \sigma_{t}^{n}} \leqslant \sum_{k \in \mathbb{Z} \cap\left[0,4 \tau_{t} n\right]}\left(\begin{array}{l}
n \\
k
\end{array}\right) e_{t}^{n}(k) \leqslant \frac{1}{\sigma_{t}^{n}} .
$$

Moreover, for every $s \in \mathbb{Z} \cap\left(4 \tau_{t} n, n\right]$ we have

$$
\sum_{m \in \mathbb{Z} \cap\left[\left(s-4 \tau_{t} n\right) / 2, s / 2\right]}\left(\begin{array}{c}
n \\
s-2 m
\end{array}\right) e_{t}^{n}(s-2 m) \geqslant \frac{1}{18 \sigma_{t}^{n}} .
$$


Proof. For simplicity of notation write $\tau=\tau_{t}$ and $\sigma=\sigma_{t}^{n}$. The rightmost inequality in (209) is an immediate consequence of (207). To establish the leftmost estimate in (209) note that by the Chernoff inequality (e.g. [2, Thm. A.1.4]) we have

$$
\sum_{k \in \mathbb{Z} \cap(4 \tau n, n]}\left(\begin{array}{l}
n \\
k
\end{array}\right) \tau^{k}(1-\tau)^{n-k}<e^{-18 \tau^{2} n \stackrel{(208)}{\leqslant}} \frac{1}{3}
$$

For every $k \in\{1, \ldots, n\}$ satisfying $k \leqslant 4 \tau n$ we have $\tau^{k}(1-\tau)^{n-k} \geqslant \sigma$, and therefore $e_{t}^{n}(k) \geqslant \frac{1}{2 \sigma} \tau^{k}(1-\tau)^{n-k}$. Hence

$$
\sum_{k \in \mathbb{Z} \cap[0,4 \tau n]}\left(\begin{array}{l}
n \\
k
\end{array}\right) e_{t}^{n}(k) \geqslant \frac{1}{2 \sigma} \sum_{k \in \mathbb{Z} \cap[0,4 \tau n]}\left(\begin{array}{l}
n \\
k
\end{array}\right) \tau^{k}(1-\tau)^{n-k} \frac{\sqrt[(211)]{>}}{2 \sigma}\left(1-\frac{1}{3}\right)=\frac{1}{3 \sigma} .
$$

This completes the proof of (209).

To prove (210), we apply a standard binomial concentration bound (e.g. [2, Cor. A.1.14]) to get the estimate

$$
\sum_{k \in \mathbb{Z} \cap[\tau n / 2,3 \tau n / 2]}\left(\begin{array}{l}
n \\
k
\end{array}\right) \tau^{k}(1-\tau)^{n-k} \geqslant 1-2 e^{-\tau n / 10} \geqslant \frac{8}{9},
$$

where in the rightmost inequality in (213) we used the assumptions (208) and $n \geqslant 8000$. Observe that for every $k \in \mathbb{Z} \cap[\tau n / 2,3 \tau n / 2]$, since by the assumption $t \in(0,1 / 4)$ we have $\tau \in(0,1 / 8)$,

$$
\frac{\left(\begin{array}{c}
n \\
k+1
\end{array}\right) \tau^{k+1}(1-\tau)^{n-k-1}}{\left(\begin{array}{l}
n \\
k
\end{array}\right) \tau^{k}(1-\tau)^{n-k}}=\frac{\tau}{1-\tau} \cdot \frac{n-k}{k+1} \in\left[\frac{1-3 \tau / 2}{3(1-\tau)}, \frac{2-\tau}{1-\tau}\right] \subseteq\left[\frac{1}{4}, 4\right] .
$$

It follows that

$$
\sum_{k \in(2 \mathbb{Z}) \cap[\tau n / 2,3 \tau n / 2]}\left(\begin{array}{l}
n \\
k
\end{array}\right) \tau^{k}(1-\tau)^{n-k} \stackrel{\sqrt{214})}{\geqslant} \frac{1}{8} \sum_{k \in \mathbb{Z} \cap[\tau n / 2,3 \tau n / 2]}\left(\begin{array}{l}
n \\
k
\end{array}\right) \tau^{k}(1-\tau)^{n-k} \stackrel{(213)}{\geqslant} \frac{1}{9},
$$

and, for the same reason,

$$
\sum_{k \in(2 \mathbb{Z}+1) \cap[\tau n / 2,3 \tau n / 2]}\left(\begin{array}{l}
n \\
k
\end{array}\right) \tau^{k}(1-\tau)^{n-k} \geqslant \frac{1}{9} .
$$

Thus,

$$
\sum_{m \in \mathbb{Z} \cap[(s-3 \tau n / 2) / 2,(s-\tau n / 2) / 2]}\left(\begin{array}{c}
n \\
s-2 m
\end{array}\right) \tau^{s-2 m}(1-\tau)^{n-(s-2 m)} \geqslant \frac{1}{9} .
$$

Finally,

$$
\begin{aligned}
& \sum_{m \in \mathbb{Z} \cap[(s-4 \tau n) / 2, s / 2]}\left(\begin{array}{c}
n \\
s-2 m
\end{array}\right) e_{t}^{n}(s-2 m) \\
& \stackrel{\frac{(207)}{\geqslant} \frac{1}{2 \sigma}}{\sum_{m \in \mathbb{Z} \cap[(s-3 \tau n / 2) / 2,(s-\tau n / 2) / 2]}}\left(\begin{array}{c}
n \\
s-2 m
\end{array}\right) \tau^{s-2 m}(1-\tau)^{n-(s-2 m) \stackrel{(215)}{\geqslant} \frac{1}{18 \sigma} .}
\end{aligned}
$$


Lemma 7.2 (Discretization of $e^{-t \Delta}$ w.r.t. Poincaré inequalities). Fix $t \in(0,1 / 4), p \in[1, \infty)$ and $n \in \mathbb{N} \cap\left[2^{13}, \infty\right)$ such that

$$
\tau_{t} \geqslant \sqrt{\frac{p \log (18 n)}{18 n}} .
$$

Let $G_{t}^{n}=\left(\mathbb{F}_{2}^{n}, E_{t}^{n}\right)$ be the graph whose vertex set is $\mathbb{F}_{2}^{n}$ and every $x, y \in \mathbb{F}_{2}^{n}$ is joined by $e_{t}^{n}\left(\|x-y\|_{1}\right)$ edges. Then the graph $G_{t}^{n}$ is $d_{t}^{n} \in \mathbb{N}$ regular, where

$$
\frac{1}{3 \sigma_{t}^{n}} \leqslant d_{t}^{n} \leqslant \frac{1}{\sigma_{t}^{n}}
$$

Moreover, for every metric space $\left(X, d_{X}\right)$ and every $f, g: \mathbb{F}_{2}^{n} \rightarrow X$ we have

$$
\begin{aligned}
\frac{1}{3\left|E_{t}^{n}\right|} \sum_{(x, y) \in E_{t}^{n}} d_{X}(f(x), g(y))^{p} \leqslant \frac{1}{2^{n}} \sum_{(x, y) \in \mathbb{F}_{2}^{n} \times \mathbb{F}_{2}^{n}}\left(e^{-t \Delta} \delta_{x}\right)(y) d_{X}(f(x), g(y))^{p} \\
\leqslant \frac{3}{\left|E_{t}^{n}\right|} \sum_{(x, y) \in E_{t}^{n}} d_{X}(f(x), g(y))^{p} .
\end{aligned}
$$

Proof. Observe that the assumptions of Lemma 7.2 imply the assumptions of Lemma 7.1, We may therefore use the conclusions of Lemma 7.1 in the ensuing proof. For simplicity of notation write $\tau=\tau_{t}$ and $\sigma=\sigma_{t}^{n}$. By definition $G_{t}^{n}$ is a regular graph. Denote its degree by $d=d_{t}^{n}$. Then,

$$
d=\sum_{k=0}^{n}\left(\begin{array}{l}
n \\
k
\end{array}\right) e_{t}(k) \stackrel{\left(\frac{207}{\epsilon}\right.}{\epsilon}\left[\frac{1}{3 \sigma}, \frac{1}{\sigma}\right] .
$$

This proves (217). We also immediately deduce the leftmost inequality in (218) as follows.

$$
\begin{aligned}
\frac{1}{2^{n}} & \sum_{(x, y) \in \mathbb{F}_{2}^{n} \times \mathbb{F}_{2}^{n}}\left(e^{-t \Delta} \delta_{x}\right)(y) d_{X}(f(x), g(y))^{p} \\
& \stackrel{(96))}{=} \frac{1}{2^{n}} \sum_{(x, y) \in \mathbb{F}_{2}^{n} \times \mathbb{F}_{2}^{n}} \tau^{\|x-y\|_{1}}(1-\tau)^{n-\|x-y\|_{1}} d_{X}(f(x), g(y))^{p} \\
& \stackrel{\frac{(207)}{\geqslant}}{\geqslant} \sum_{(x, y) \in \mathbb{F}_{2}^{n} \times \mathbb{F}_{2}^{n}} e_{t}^{n}\left(\|x-y\|_{1}\right) d_{X}(f(x), g(y))^{p} \\
& \frac{1}{\geqslant} \frac{1}{3\left|E_{t}^{n}\right|} \sum_{(x, y) \in E_{t}^{n}} d_{X}(f(x), g(y))^{p}
\end{aligned}
$$

where we used the fact that $\left|E_{n}^{t}\right|=2^{n} d$.

It remains to prove the rightmost inequality in (218). To this end fix $k \in \mathbb{Z}$ satisfying $0 \leqslant k \leqslant 4 \tau n$ and $m \in \mathbb{N} \cup\{0\}$ satisfying $k+2 m \leqslant n$. For every permutation $\pi \in S_{n}$ define $z_{0}^{\pi}, \ldots, z_{2 m+1}^{\pi}, y_{0}^{\pi}, \ldots, y_{2 m+1}^{\pi} \in \mathbb{F}_{2}^{n}$ by setting $z_{0}^{\pi}=y_{0}^{\pi}=0$ and for $i \in\{1, \ldots, 2 m+1\}$,

$$
z_{i}^{\pi} \stackrel{\text { def }}{=} \sum_{j=1}^{k-1} e_{\pi(j)}+e_{\pi(k+i-1)},
$$


and

$$
y_{i}^{\pi} \stackrel{\text { def }}{=} \sum_{j=1}^{i} z_{j}^{\pi}
$$

where the sum in (220) is performed in $\mathbb{F}_{2}^{n}$ (i.e., modulo 2), and we recall that $e_{1}, \ldots, e_{n}$ is the standard basis of $\mathbb{F}_{2}^{n}$. For every $x \in \mathbb{F}_{2}^{n}$ we have

$$
\begin{aligned}
& d_{X}\left(f(x), g\left(x+y_{2 m+1}^{\pi}\right)\right) \\
& \quad \leqslant \sum_{i=0}^{m} d_{X}\left(f\left(x+y_{2 i}^{\pi}\right), g\left(x+y_{2 i+1}^{\pi}\right)\right)+\sum_{i=0}^{m-1} d_{X}\left(g\left(x+y_{2 i+1}^{\pi}\right), f\left(x+y_{2 i+2}^{\pi}\right)\right) .
\end{aligned}
$$

Hence, Hölder's inequality yields the following estimate.

$$
\begin{aligned}
& \frac{d_{X}\left(f(x), g\left(x+y_{2 m+1}^{\pi}\right)\right)^{p}}{(2 m+1)^{p-1}} \\
& \quad \leqslant \sum_{i=0}^{m} d_{X}\left(f\left(x+y_{2 i}^{\pi}\right), g\left(x+y_{2 i+1}^{\pi}\right)\right)^{p}+\sum_{i=0}^{m-1} d_{X}\left(g\left(x+y_{2 i+1}^{\pi}\right), f\left(x+y_{2 i+2}^{\pi}\right)\right)^{p} .
\end{aligned}
$$

Note that

$$
y_{2 m+1}^{\pi}=\sum_{j=1}^{k+2 m} e_{\pi(j)}
$$

Therefore, if $\pi \in S_{n}$ is chosen uniformly at random then $y_{2 m+1}^{\pi}$ is distributed uniformly over the $\left(\begin{array}{c}n \\ k+2 m\end{array}\right)$ elements $w \in \mathbb{F}_{2}$ with $\|w\|_{1}=k+2 m$. This observation implies that

$$
\frac{1}{2^{n} n !} \sum_{x \in \mathbb{F}_{2}^{n}} \sum_{\pi \in S_{n}} d_{X}\left(f(x), g\left(x+y_{2 m+1}^{\pi}\right)\right)^{p}=\frac{1}{2^{n}\left(\begin{array}{c}
n \\
k+2 m
\end{array}\right)} \sum_{\substack{(x, y) \in \mathbb{F}_{2}^{n} \times \mathbb{F}_{2}^{n} \\
\|x-y\|_{1}=k+2 m}} d_{X}(f(x), g(y))^{p} .
$$

Similarly, for every $j \in\{0, \ldots, 2 m\}$ we have

$$
\begin{array}{r}
\sum_{x \in \mathbb{F}_{2}^{n}} \sum_{\pi \in S_{n}} d_{X}\left(f\left(x+y_{j}^{\pi}\right), g\left(x+y_{j+1}^{\pi}\right)\right)^{p}=\sum_{\pi \in S_{n}} \sum_{x \in \mathbb{F}_{2}^{n}} d_{X}\left(f\left(x+y_{j}^{\pi}\right), g\left(x+y_{j}^{\pi}+z_{j}^{\pi}\right)\right)^{p} \\
=\sum_{\pi \in S_{n}} \sum_{u \in \mathbb{F}_{2}^{n}} d_{X}\left(f(u), g\left(u+z_{j}^{\pi}\right)\right)^{p}=\frac{n !}{\left(\begin{array}{l}
n \\
k
\end{array}\right)} \sum_{\substack{(u, v) \in \mathbb{F}_{2}^{n} \times \mathbb{F}_{2}^{n} \\
\|u-v\|_{1}=k}} d_{X}(f(u), g(v))^{p},
\end{array}
$$

where in the penultimate equality of (223) we used the fact that for each $\pi \in S_{n}$, if $x$ is chosen uniformly at random from $\mathbb{F}_{2}^{n}$ then $x+y_{j}^{\pi}$ is distributed uniformly over $\mathbb{F}_{2}^{n}$, and in the last equality of (223) we used the fact that, because $\left\|z_{j}^{\pi}\right\|_{1}=k$, if $\pi \in S_{n}$ is chosen uniformly at random then $z_{j}^{\pi}$ is distributed uniformly over the $\left(\begin{array}{l}n \\ k\end{array}\right)$ elements $w \in \mathbb{F}_{2}$ with $\|w\|_{1}=k$.

A combination of (221), (222) and (223) yields the following (crude) estimate.

$$
\frac{1}{2^{n}\left(\begin{array}{c}
n \\
k+2 m
\end{array}\right)} \sum_{\substack{(x, y) \in \mathbb{F}_{2}^{n} \times \mathbb{F}_{2}^{n} \\
\|x-y\|_{1}=k+2 m}} d_{X}(f(x), g(y))^{p} \leqslant \frac{n^{p}}{2^{n}\left(\begin{array}{l}
n \\
k
\end{array}\right)} \sum_{\substack{(x, y) \in \mathbb{F}_{2}^{n} \times \mathbb{F}_{2}^{n} \\
\|x-y\|_{1}=k}} d_{X}(f(x), g(y))^{p} .
$$


If we fix $s \in \mathbb{N} \cap(4 \tau n, n]$ then (224) implies that for every $m \in \mathbb{N} \cap[(s-4 \tau n) / 2, s / 2]$,

$$
\frac{\left(\begin{array}{c}
n \\
s-2 m
\end{array}\right)}{n^{p}\left(\begin{array}{l}
n \\
s
\end{array}\right)} \sum_{\substack{(x, y) \in \mathbb{F}_{2}^{n} \times \mathbb{F}_{2}^{n} \\
\|x-y\|_{1}=s}} d_{X}(f(x), g(y))^{p} \leqslant \sum_{\substack{(x, y) \in \mathbb{F}_{2}^{n} \times \mathbb{F}_{2}^{n} \\
\|x-y\|_{1}=s-2 m}} d_{X}(f(x), g(y))^{p} .
$$

Multiplying both sides of (225) by $e_{t}^{n}(s-2 m)$ and summing over $m \in \mathbb{N} \cap[(s-4 \tau n) / 2, s / 2]$ yields the following estimate.

$$
\begin{aligned}
& \frac{\sum_{m \in \mathbb{Z} \cap[(s-4 \tau n) / 2, s / 2]}\left(\begin{array}{c}
n \\
s-2 m
\end{array}\right) e_{t}^{n}(s-2 m)}{n^{p}\left(\begin{array}{c}
n \\
s
\end{array}\right)} \sum_{\substack{x, y \in \mathbb{F}_{2}^{n} \\
\|x-y\|_{1}=s}} d_{X}(f(x), g(y))^{p} \\
& \leqslant \sum_{m \in \mathbb{Z} \cap[(s-4 \tau n) / 2, s / 2]} e_{t}^{n}(s-2 m) \sum_{\substack{(x, y) \in \mathbb{F}_{2}^{n} \times \mathbb{F}_{2}^{n} \\
\|x-y\|_{1}=s-2 m}} d_{X}(f(x), g(y))^{p} \leqslant \sum_{(x, y) \in E_{t}^{n}} d_{X}(f(x), g(y))^{p} .
\end{aligned}
$$

Due to (210) it follows that for every $s \in \mathbb{N} \cap(4 \tau n, n]$ we have

$$
\frac{1}{\left(\begin{array}{l}
n \\
s
\end{array}\right)} \sum_{\substack{(x, y) \in \mathbb{F}_{2}^{n} \times \mathbb{F}_{2}^{n} \\
\|x-y\|_{1}=s}} d_{X}(f(x), g(y))^{p} \leqslant 18 \sigma n^{p} \sum_{(x, y) \in E_{t}^{n}} d_{X}(f(x), g(y))^{p} .
$$

Now,

$$
\begin{aligned}
& \frac{1}{2^{n}} \sum_{(x, y) \in \mathbb{F}_{2}^{n} \times \mathbb{F}_{2}^{n}}\left(e^{-t \Delta} \delta_{x}\right)(y) d_{X}(f(x), g(y))^{p} \\
& \stackrel{196)}{=} \quad \frac{1}{2^{n}} \sum_{s=0}^{n} \tau^{s}(1-\tau)^{n-s} \sum_{\substack{(x, y) \in \mathbb{F}_{2}^{n} \times \mathbb{F}_{2}^{n} \\
\|x-y\|_{1}=s}} d(f(x), g(y))^{p} \\
& \stackrel{(207) \wedge(226)}{\leqslant} \frac{\sigma}{2^{n}}\left(2+18 n^{p} \sum_{s \in \mathbb{Z} \cap(4 \tau n, n]}\left(\begin{array}{l}
n \\
s
\end{array}\right) \tau^{s}(1-\tau)^{n-s}\right) \sum_{(x, y) \in E_{t}^{n}} d_{X}(f(x), g(y))^{p} \\
& \stackrel{(211) \wedge(219)}{\leqslant}\left(2+18 n^{p} e^{-18 \tau^{2} n}\right) \frac{1}{d 2^{n}} \sum_{(x, y) \in E_{t}^{n}} d_{X}(f(x), g(y))^{p} \\
& \stackrel{3}{\leqslant} \quad \frac{3}{\left|E_{t}^{n}\right|} \sum_{(x, y) \in E_{t}^{n}} d_{X}(f(x), g(y))^{p} .
\end{aligned}
$$

This concludes the proof of (218).

In what follows for every $n \in \mathbb{N}$ we fix $V_{n} \subseteq \mathbb{F}_{2}^{n}$ which is a "good linear code", i.e., a linear subspace over $\mathbb{F}_{2}$ with

$$
D_{n} \stackrel{\text { def }}{=} \operatorname{dim}\left(V_{n}\right) \geqslant \frac{n}{10} \quad \text { and } \quad k_{n} \stackrel{\text { def }}{=} \min _{x \in V_{n} \backslash\{0\}}\|x\|_{1} \geqslant \frac{n}{10} .
$$

Also, we assume that the sequences $\left\{D_{n}\right\}_{n=1}^{\infty}$ and $\left\{k_{n}\right\}_{n=1}^{\infty}$ are increasing. The essentially arbitrary choice of the constant 10 in (227) does not play an important role in what follows. 
The fact that $\left\{V_{n}\right\}_{n=1}^{\infty}$ exists is simple; see [37]. We shall use the standard notation

$$
V_{n}^{\perp} \stackrel{\text { def }}{=}\left\{x \in \mathbb{F}_{2}^{n}: \forall y \in V_{n}, \quad \sum_{j=1}^{n} x_{j} y_{j} \equiv 0 \quad \bmod 2\right\} .
$$

Lemma 7.3. For every $K, p \in(1, \infty)$ there exists $n(K, p) \in \mathbb{N}$ and $\delta(K, p) \in(0,1)$ with the following properties. Setting

$$
m_{n} \stackrel{\text { def }}{=}\left|\mathbb{F}_{2}^{n} / V_{n}^{\perp}\right| \stackrel{(227)}{=} 2^{D_{n}}
$$

there exists a sequence of connected regular graphs

$$
\left\{H_{n}(K, p)\right\}_{n=n(K, p)}^{\infty}
$$

such that for every integer $n \geqslant n(K, p)$ the graph $H_{n}(K, p)$ has $m_{n}$ vertices and degree

$$
d_{n}(K, p) \leqslant e^{\left(\log m_{n}\right)^{1-\delta(K, p)}},
$$

and for every $K$-convex Banach space $X=\left(X,\|\cdot\|_{X}\right)$ with $K(X) \leqslant K$,

$$
\forall n \in[n(K, p), \infty) \cap \mathbb{N}, \quad \gamma_{+}\left(\left(H_{n}(K, p),\|\cdot\|_{X}^{p}\right) \leqslant 9^{p+1} .\right.
$$

Proof. Fix $K, p \in(1, \infty)$. Let $A=A(K, p), B=B(K, p), C=C(K, p)$ be the constants of Theorem 5.1. Recall that $B>2$. Set

$$
t=t(n, K, p) \stackrel{\text { def }}{=}\left(\frac{\log (2 C)}{k_{n} A}\right)^{1 / B},
$$

where $k_{n}$ is given in (227). Then there exists $n(K, p) \in \mathbb{N}$ such that every integer $n \geqslant n(K, p)$ satisfies the assumptions of Lemma 7.2, and moreover there exists $\delta(K, p) \in(0,1)$ such that for every integer $n \geqslant n(K, p)$ we have

$$
\frac{1}{\tau_{t}^{8 n \tau_{t}}} \leqslant e^{\left(\log m_{n}\right)^{1-\delta(K, p)}} .
$$

(To verify (232) recall that $\log m_{n}=D_{n} \log 2 \geqslant n / 20$.)

Assume from now on that $n \in \mathbb{N}$ satisfies $n \geqslant n(K, p)$. Let $G_{t}^{n}=\left(\mathbb{F}_{2}^{n}, E_{t}^{n}\right)$ be the graph constructed in Lemma 7.2. The degree of $G_{t}^{n}$ is

$$
d_{t}^{n} \stackrel{(217)}{\leqslant} \frac{1}{\sigma_{t}^{n}} \stackrel{(206)}{\leqslant} \frac{1}{\tau_{t}^{8 n \tau_{t}}} \stackrel{(232)}{\leqslant} e^{n^{1-\delta(K, p)}} .
$$

The desired graph $H_{n}=H_{n}(K, p)$ is defined to be the following quotient of $G_{t}^{n}$. The vertex set of $H_{n}$ is $\mathbb{F}_{2}^{n} / V_{n}^{\perp}$. Given two cosets $x+V_{n}^{\perp}, y+V_{n}^{\perp} \in \mathbb{F}_{2}^{n} / V_{n}^{\perp}$, the number of edges joining $x+V_{n}^{\perp}$ and $y+V_{n}^{\perp}$ in $H_{n}$ is defined to be the number of edges of $G_{t}^{n}$ with one endpoint in $x+V_{n}^{\perp}$ and the other endpoint in $y+V_{n}^{\perp}$, divided by the cardinality of $V_{n}^{\perp}$. Thus, the number of edges joining $x+V_{n}^{\perp}$ and $y+V_{n}^{\perp}$ in the graph $H_{n}$ equals

$$
\frac{1}{\left|V_{n}^{\perp}\right|} \sum_{\left(u^{\perp}, v^{\perp}\right) \in V_{n}^{\perp} \times V_{n}^{\perp}} e_{t}^{n}\left(\left\|x-y+\left(u^{\perp}-v^{\perp}\right)\right\|_{1}\right)=\sum_{u^{\perp} \in V_{n}^{\perp}} e_{t}^{n}\left(\left\|x-y+u^{\perp}\right\|_{1}\right) .
$$

Hence $H_{n}$ is a regular graph of the same degree as $G_{t}^{n}$ (i.e., the degree of $H_{n}$ equals $d_{t}^{n}$ ). In what follows we let $\pi: \mathbb{F}_{2}^{n} \rightarrow \mathbb{F}_{2}^{n} / V_{n}^{\perp}$ denote the quotient map.

Fix a $K$-convex Banach space $\left(X,\|\cdot\|_{X}\right)$ with $K(X) \leqslant K$. For every $f \in L_{p}\left(\mathbb{F}_{2}^{n} / V_{n}^{\perp}, X\right)$ define $\pi f: \mathbb{F}_{2}^{n} \rightarrow X$ by $\pi f(x)=f(\pi(x))$. Thus $\pi f$ is constant on the cosets of $V_{n}^{\perp}$. It follows 
from [27, Lem. 3.3] that if $\sum_{x \in \mathbb{F}_{2}^{n} / V_{n}^{\perp}} f(x)=0$ then $\pi f \in L_{p}^{\geqslant k_{n}}\left(\mathbb{F}_{2}^{n}, X\right)$, where $k_{n}$ is defined in (227). By Theorem 5.1 we therefore have

$$
\frac{\left\|\left(e^{-t \Delta} \pi\right) f\right\|_{L_{p}\left(\mathbb{F}_{2}^{n} / V_{n}^{\perp}, X\right)}}{\|f\|_{L_{p}\left(\mathbb{F}_{2}^{n} / V_{n}^{\perp}, X\right)}} \leqslant C e^{-A k_{n} \min \left\{t, t^{B}\right\}} \stackrel{(231)}{=} \frac{1}{2} .
$$

Let $Q$ be the $\left(\mathbb{F}_{2}^{n} / V_{n}^{\perp}\right) \times\left(\mathbb{F}_{2}^{n} / V_{n}^{\perp}\right)$ symmetric stochastic matrix corresponding to the averaging operator $e^{-t \Delta} \pi$, i.e., the entry of $Q$ at $\left(x+V_{n}^{\perp}, y+V_{n}^{\perp}\right) \in\left(\mathbb{F}_{2}^{n} / V_{n}^{\perp}\right) \times\left(\mathbb{F}_{2}^{n} / V_{n}^{\perp}\right)$ is

$$
q_{x+V_{n}^{\perp}, y+V_{n}^{\perp}} \stackrel{\text { def }}{=}\left(\left(e^{-t \Delta} \pi\right) \delta_{x+V_{n}^{\perp}}\right)\left(y+V_{n}^{\perp}\right)=\sum_{\substack{u \in a+V_{n}^{\perp} \\ v \in b+V_{n}^{\perp}}} \tau_{t}^{\|a-b\|_{1}}\left(1-\tau_{t}\right)^{n-\|a-b\|_{1}} .
$$

Since (233) holds for all $f \in L_{p}\left(\mathbb{F}_{2}^{n} / V_{n}^{\perp}, X\right)$ with $\sum_{x \in \mathbb{F}_{2}^{n} / V_{n}^{\perp}} f(x)=0$, we have $\lambda_{X}^{(p)}(Q) \leqslant \frac{1}{2}$ (recall here the notation introduced in (153) $)$. Consequently, Lemma 6.1 implies that

$$
\gamma_{+}\left(Q,\|\cdot\|_{X}^{p}\right) \leqslant 9^{p} .
$$

Thus every $f, g: \mathbb{F}_{2}^{n} / V_{n}^{\perp} \rightarrow X$ satisfy

$$
\begin{aligned}
\frac{1}{\left|\mathbb{F}_{2}^{n} / V_{n}^{\perp}\right|^{2}} \sum_{(S, T) \in\left(\mathbb{F}_{2}^{n} / V_{n}^{\perp}\right) \times\left(\mathbb{F}_{2}^{n} / V_{n}^{\perp}\right)}\|f(S)-g(T)\|_{X}^{p} \\
\leqslant \frac{9^{p}}{\left|\mathbb{F}_{2}^{n} / V_{n}^{\perp}\right|} \sum_{(S, T) \in\left(\mathbb{F}_{2}^{n} / V_{n}^{\perp}\right) \times\left(\mathbb{F}_{2}^{n} / V_{n}^{\perp}\right)} q_{S, T}\|f(S)-g(T)\|_{X}^{p} .
\end{aligned}
$$

Observe that

$$
\begin{aligned}
& \sum_{(S, T) \in\left(\mathbb{F}_{2}^{n} / V_{n}^{\perp}\right) \times\left(\mathbb{F}_{2}^{n} / V_{n}^{\perp}\right)} q_{S, T}\|f(S)-g(T)\|_{X}^{p} \stackrel{\text { (234) }}{=} \sum_{(a, b) \in \mathbb{F}_{2}^{n} \times \mathbb{F}_{2}^{n}}\left(e^{-t \Delta} \delta_{a}\right)(b)\|\pi f(a)-\pi g(b)\|_{X}^{p} \\
& \stackrel{\frac{218}{\leqslant}}{\leqslant} \frac{3}{\left|E_{t}^{n}\right|} \sum_{(a, b) \in E_{t}^{n}}\|\pi f(a)-\pi g(b)\|_{X}^{p} \\
& =\frac{3}{2^{n} d_{t}^{n}} \sum_{(S, T) \in\left(\mathbb{F}_{2}^{n} / V_{n}^{\perp}\right) \times\left(\mathbb{F}_{2}^{n} / V_{n}^{\perp}\right)}\left(\sum_{(a, b) \in S \times T} E_{t}^{n}(a, b)\right)\|f(S)-g(T)\|_{X}^{p} \\
& =\frac{3}{\left|E\left(H_{n}\right)\right|} \sum_{(S, T) \in E\left(H_{n}\right)}\|f(S)-g(T)\|_{X}^{p} \cdot
\end{aligned}
$$

In (236) we used the fact that for every $S, T \in \mathbb{F}_{2}^{n} / V_{n}^{\perp}$, by the definition of the graph $H_{n}$, the quantity

$$
\frac{1}{\left|V_{n}^{\perp}\right|} \sum_{(a, b) \in S \times T} E_{t}^{n}(a, b)
$$

equals the number of edges joining $S$ and $T$ in $H_{n}$, and that since $H_{n}$ is a $d_{t}^{n}$-regular graph we have $\left|V_{n}^{\perp}\right| /\left(2^{n} d_{t}^{n}\right)=1 /\left|E\left(H_{n}\right)\right|$.

The desired estimate (230) now follows from (235) and (236).

The case $p=2$ of Corollary 7.4 below (which is nothing more than a convenient way to restate Lemma 7.3) corresponds to Lemma 1.12 . 
Corollary 7.4. For every $\delta \in(0,1)$ and $p \in(1, \infty)$ there exists $n_{0}^{p}(\delta) \in \mathbb{N}$ and a sequence of regular graphs $\left\{H_{n}^{p}(\delta)\right\}_{n=n_{0}^{p}(\delta)}^{\infty}$ such that for every every $n \geqslant n_{0}^{p}(\delta)$ the graph $H_{n}^{p}(\delta)$ is regular and has $m_{n}$ vertices, with $m_{n}$ given in (228). The degree of $H_{n}^{p}(\delta)$, denoted $d_{n}^{p}(\delta)$, satisfies

$$
d_{n}^{p}(\delta) \leqslant e^{\left(\log m_{n}\right)^{1-\delta}} .
$$

Moreover, for every $K$-convex Banach space $\left(X,\|\cdot\|_{X}\right)$ we have $\gamma_{+}\left(H_{n}^{p}(\delta),\|\cdot\|_{X}^{p}\right)<\infty$ for all integers $n \geqslant n_{0}^{p}(\delta)$, and there exists $\delta_{0}^{p}(X) \in(0,1)$ such that for every $0<\delta \leqslant \delta_{0}^{p}(X)$ and every integer $n \geqslant n_{0}^{p}(\delta)$ we have

$$
\gamma_{+}\left(H_{n}^{p}(\delta),\|\cdot\|_{X}^{p}\right) \leqslant 9^{p+1}
$$

Proof. We shall use here the notation of Lemma 7.3. We may assume without loss of generality that $\delta(K, p)$ decreases continuously with $K$ and that $\lim _{K \rightarrow \infty} \delta(K, p)=0$. If $\delta \in(\delta(2, p), 1)$ then let $n_{0}^{p}(\delta)$ be the smallest integer such that $\left(\log m_{n}\right)^{1-\delta} \geqslant \log 3$ and set $H_{n}^{p}(\delta)=C_{m_{n}}^{\circ}$ be the $m_{n}$-cycle with self loops. Since in this case $d_{n}^{p}(\delta)=3$, the desired degree bound (237) holds true by design. Moreover, in this case the finiteness of $\gamma_{+}\left(H_{n}^{p}(\delta),\|\cdot\|_{X}^{p}\right)$ is a consequence of Lemma 2.1. For $\delta \in(0, \delta(2, p)]$ we can define $K_{\delta}^{p}=\sup \{K \in[2, \infty): \delta(K, p) \geqslant \delta\}$. Set $n_{0}^{p}(\delta)=n\left(K_{\delta}^{p}, p\right)$ and for every integer $n \geqslant n_{0}^{p}(\delta)$ define $H_{n}^{p}(\delta)=H_{n}\left(K_{\delta}^{p}, p\right)$. Thus $d_{n}^{p}(\delta)=d_{n}\left(K_{\delta}^{p}, p\right)$ and (237) follows from (229). Finally, setting $\delta_{0}^{p}(X)=\inf \left\{\delta \in(0, \delta(2, p)]: K_{\delta}^{p} \leqslant 2 K(X)\right\}$, it follows that for every $\delta \in\left(0, \delta_{0}^{p}(X)\right]$ we have $K_{\delta}^{p} \geqslant 2 K(X)$, so that (238) follows from (230).

Remark 7.5. In Remark 5.12 we asked whether Theorem 5.10 can be improved so as to yield the estimate

$$
\|\Delta f\|_{L_{p}\left(\mathbb{F}_{2}^{n}, X\right)} \gtrsim_{X, p} k\|f\|_{L_{p}\left(\mathbb{F}_{2}^{n}, X\right)}
$$

for every $f \in L_{p}^{\geqslant k}\left(\mathbb{F}_{2}^{n}, X\right)$. Here $\left(X,\|\cdot\|_{X}\right)$ is a $K$-convex Banach space and the implied constant is allowed to depend only on $p \in(1, \infty)$ and the $K$-convexity constant $K(X)$. If true, this would yield the following simpler proof of Lemma 7.3, with better degree bounds. Continuing to use the notation of Lemma [7.3, we would consider instead the "vanilla" quotient graph $G$ on $\mathbb{F}_{2}^{n} / V_{n}^{\perp}$, i.e., the graph in which the number of edges joining two cosets $x+V_{n}^{\perp}, y+V_{n}^{\perp}$ equals the number of standard hypercube edges joining these two sets divided by $\left|V_{n}^{\perp}\right|$. The degree of this graph is $n \asymp \log m_{n}$. Given a mean-zero $f: \mathbb{F}_{2}^{n} / V_{n}^{\perp} \rightarrow X$ we think of $f$ as being a $V_{n}^{\perp}$-invariant function defined on $\mathbb{F}_{2}^{n}$, in which case by [27, Lem. 3.3] we have $f \in L_{p}^{\geqslant k_{n}}\left(\mathbb{F}_{2}^{n}, X\right)$, where $k_{n} \asymp n$ is given in (227). Assuming the validity of (239),

$$
\begin{aligned}
n\|f\|_{L_{p}\left(\mathbb{F}_{2}^{n}, X\right)} \lesssim k_{n}\|f\|_{L_{p}\left(\mathbb{F}_{2}^{n}, X\right)} \lesssim X, p & \|\Delta f\|_{L_{p}\left(\mathbb{F}_{2}^{n}, X\right)} \\
=\left\|\sum_{i=1}^{n} \partial_{i} f\right\|_{L_{p}\left(\mathbb{F}_{2}^{n}, X\right)} & \leqslant \sum_{i=1}^{n}\left\|\partial_{i} f\right\|_{L_{p}\left(\mathbb{F}_{2}^{n}, X\right)} \leqslant n^{1-1 / p}\left(\sum_{i=1}^{n}\left\|\partial_{i} f\right\|_{L_{p}\left(\mathbb{F}_{2}^{n}, X\right)}^{p}\right)^{1 / p} .
\end{aligned}
$$

It follows that

$$
\frac{1}{2^{2 n}} \sum_{(x, y) \in \mathbb{F}_{2}^{n} \times \mathbb{F}_{2}^{n}}\|f(x)-f(y)\|_{X}^{p} \leqslant 2^{p}\|f\|_{L_{p}\left(\mathbb{F}_{2}^{n}, X\right)}^{p} \stackrel{(240)}{\lesssim X, p} \frac{1}{n} \sum_{i=1}^{n}\left\|\partial_{i} f\right\|_{L_{p}\left(\mathbb{F}_{2}^{n}, X\right)}^{p} .
$$

By the definition of the quotient graph $G$, it follows from (241) that $\gamma(G, X) \lesssim_{p, X} 1$. Using Lemma 2.6 we conclude that there exists a regular graph $G^{\prime}$ with $m_{n} / 2=2^{D_{n}-1}$ vertices and degree at most a constant multiple of $\log m_{n}$ such that $\gamma_{+}\left(G^{\prime}, X\right) \lesssim_{p, X} 1$. 


\section{GRAPH PRODUCTS}

The purpose of this section is to recall the definitions of the various graph products that were mentioned in the introduction, and to prove Theorem 1.13 .

8.1. Sub-multiplicativity for tensor products. The case of tensor products, i.e., part (II) of Theorem 1.13, is very simple, and should mainly serve as warmup for the other parts of Theorem 1.13.

Proposition 8.1 (Sub-multiplicativity for tensor products). Fix $m, n \in \mathbb{N}$. Let $A=\left(a_{i j}\right)$ be an $m \times m$ symmetric stochastic matrix and let $B=\left(b_{i j}\right)$ be an $n \times n$ symmetric stochastic matrix. Then every kernel $K: X \times X \rightarrow[0, \infty)$ satisfies

$$
\gamma_{+}(A \otimes B, K) \leqslant \gamma_{+}(A, K) \gamma_{+}(B, K) .
$$

Proof. Fix $f, g:\{1, \ldots, m\} \times\{1, \ldots, n\} \rightarrow X$. Then for every fixed $s, t \in\{1, \ldots, n\}$,

$$
\frac{1}{m^{2}} \sum_{i=1}^{m} \sum_{j=1}^{m} K(f(i, s), g(j, t)) \leqslant \frac{\gamma_{+}(A, K)}{m} \sum_{i=1}^{m} \sum_{j=1}^{m} a_{i j} K(f(i, s), g(j, t)) \text {. }
$$

Also, for every fixed $i, j \in\{1, \ldots, m\}$ we have

$$
\frac{1}{n^{2}} \sum_{s=1}^{m} \sum_{t=1}^{m} K(f(i, s), g(j, t)) \leqslant \frac{\gamma_{+}(B, K)}{n} \sum_{s=1}^{n} \sum_{t=1}^{n} b_{s t} K(f(i, s), g(j, t)) .
$$

Consequently,

$$
\begin{aligned}
& \frac{1}{m^{2} n^{2}} \sum_{i=1}^{m} \sum_{j=1}^{m} \sum_{s=1}^{n} \sum_{t=1}^{n} K(f(i, s), g(j, t))=\frac{1}{n^{2}} \sum_{s=1}^{n} \sum_{t=1}^{n} \frac{1}{m^{2}} \sum_{i=1}^{m} \sum_{j=1}^{m} K(f(i, s), g(j, t)) \\
& \stackrel{\text { (243) }}{\leqslant} \frac{1}{n^{2}} \sum_{s=1}^{n} \sum_{t=1}^{n} \frac{\gamma_{+}(A, K)}{m} \sum_{i=1}^{m} \sum_{j=1}^{m} a_{i j} K(f(i, s), g(j, t)) \\
& =\frac{\gamma_{+}(A, K)}{m} \sum_{i=1}^{m} \sum_{j=1}^{m} a_{i j} \frac{1}{n^{2}} \sum_{s=1}^{m} \sum_{t=1}^{m} K(f(i, s), g(j, t)) \\
& \stackrel{(244)}{\leqslant} \frac{\gamma_{+}(A, K)}{m} \sum_{i=1}^{m} \sum_{j=1}^{m} a_{i j} \frac{\gamma_{+}(B, K)}{n} \sum_{s=1}^{n} \sum_{t=1}^{n} b_{s t} K(f(i, s), g(j, t)) \\
& =\frac{\gamma_{+}(A, K) \gamma_{+}(B, K)}{m n} \sum_{i=1}^{m} \sum_{j=1}^{m} \sum_{s=1}^{n} \sum_{t=1}^{n}(A \otimes B)_{i j s t} K(f(i, s), g(j, t)) .
\end{aligned}
$$

Since (245) holds for every $f, g:\{1, \ldots, n\} \times\{1, \ldots, m\} \rightarrow X$, (242) follows.

This concludes the proof of part (II) of Theorem 1.13. Nevertheless, when the kernel in question is the $p$ th power of a norm whose modulus of convexity has power type $p$ it is possible improve Proposition 8.1 as follows.

Lemma 8.2. Fix $m, n \in \mathbb{N}$ and $p \in[2, \infty)$. Let $A=\left(a_{i j}\right)$ be an $m \times m$ symmetric stochastic matrix and let $B=\left(b_{i j}\right)$ be an $n \times n$ symmetric stochastic matrix. Suppose that $\left(X,\|\cdot\|_{X}\right)$ is a Banach space that satisfies the p-uniform convexity inequality (163). Then

$$
\gamma_{+}\left(A \otimes B,\|\cdot\|_{X}^{p}\right) \leqslant 2^{p-1} \max \left\{\gamma_{+}\left(A,\|\cdot\|_{X}^{p}\right),\left(2^{p-1}-1\right) K_{p}(X)^{p} \gamma_{+}\left(B,\|\cdot\|_{X}^{p}\right)\right\} .
$$


Proof. For simplicity of notation write

$$
c \stackrel{\text { def }}{=} \frac{1}{\left(2^{p-1}-1\right) K_{p}(X)^{p}}
$$

and

$$
\Gamma \stackrel{\text { def }}{=} 2^{p-1} \max \left\{\gamma_{+}\left(A,\|\cdot\|_{X}^{p}\right), \frac{1}{c} \gamma_{+}\left(B,\|\cdot\|_{X}^{p}\right)\right\} .
$$

Fix $f, g:\{1, \ldots, m\} \times\{1, \ldots, n\} \rightarrow X$. For every $i, j \in\{1, \ldots, m\}$ and $s \in\{1, \ldots, n\}$ consider the $X$-valued random variable $U_{i j}^{s}$ which, for every $t \in\{1, \ldots, m\}$, takes the value $f(i, s)-g(j, t)$ with probability $b_{s t}$. An application of Lemma 6.5 with $U=U_{i j}^{s}$ shows that if for every $j \in\{1, \ldots, m\}$ and $s \in\{1, \ldots, n\}$ we define

$$
h(j, s) \stackrel{\text { def }}{=} \sum_{t=1}^{n} b_{s t} g(j, t),
$$

then for every $i, j \in\{1, \ldots, m\}$ and $s \in\{1, \ldots, n\}$ we have

$$
\|f(i, s)-h(j, s)\|_{X}^{p}+c \sum_{t=1}^{n} b_{s t}\|h(j, s)-g(j, t)\|_{X}^{p} \leqslant \sum_{t=1}^{n} b_{s t}\|f(i, s)-g(j, t)\|_{X}^{p} .
$$

By the definition of $\gamma_{+}\left(A,\|\cdot\|_{X}^{p}\right)$, for every fixed $s \in\{1, \ldots, n\}$ we have

$$
\frac{1}{m^{2}} \sum_{i=1}^{m} \sum_{j=1}^{m}\|f(i, s)-h(j, s)\|_{X}^{p} \leqslant \frac{\gamma_{+}\left(A,\|\cdot\|_{X}^{p}\right)}{m} \sum_{i=1}^{m} \sum_{j=1}^{m} a_{i j}\|f(i, s)-h(j, s)\|_{X}^{p},
$$

Similarly, for every fixed $j \in\{1, \ldots, m\}$ we have

$$
\frac{1}{n^{2}} \sum_{s=1}^{n} \sum_{t=1}^{n}\|h(j, s)-g(j, t)\|_{X}^{p} \leqslant \frac{\gamma_{+}\left(B,\|\cdot\|_{X}^{p}\right)}{n} \sum_{s=1}^{n} \sum_{t=1}^{n} b_{s t}\|h(j, s)-g(j, t)\|_{X}^{p} .
$$

By the triangle inequality, for every fixed $i, j \in\{1, \ldots, m\}$ and $s \in\{1, \ldots, n\}$ we have

$$
\frac{1}{n} \sum_{t=1}^{n}\|f(i, s)-g(j, t)\|_{X}^{p} \leqslant 2^{p-1}\|f(i, s)-h(j, s)\|_{X}^{p}+\frac{2^{p-1}}{n} \sum_{t=1}^{n}\|h(j, s)-g(j, t)\|_{X}^{p} .
$$

By averaging (251) over $i, j \in\{1, \ldots, m\}$ and $s \in\{1, \ldots, n\}$ we deduce that

$$
\begin{aligned}
& \frac{1}{m^{2} n^{2}} \sum_{i=1}^{m} \sum_{j=1}^{m} \sum_{s=1}^{n} \sum_{t=1}^{n}\|f(i, s)-g(j, t)\|_{X}^{p} \\
\leqslant & \frac{2^{p-1}}{n} \sum_{s=1}^{n} \frac{1}{m^{2}} \sum_{i=1}^{m} \sum_{j=1}^{m}\|f(i, s)-h(j, s)\|_{X}^{p}+\frac{2^{p-1}}{m} \sum_{j=1}^{m} \frac{1}{n^{2}} \sum_{s=1}^{n} \sum_{t=1}^{n}\|h(j, s)-g(j, t)\|_{X}^{p} .
\end{aligned}
$$


By substituting (249) and (250) into (252) we obtain the estimate

$$
\begin{aligned}
& \frac{1}{m^{2} n^{2}} \sum_{i=1}^{m} \sum_{j=1}^{m} \sum_{s=1}^{n} \sum_{t=1}^{n}\|f(i, s)-g(j, t)\|_{X}^{p} \\
& \leqslant \frac{2^{p-1} \gamma_{+}\left(A,\|\cdot\|_{X}^{p}\right)}{m n} \sum_{i=1}^{m} \sum_{j=1}^{m} \sum_{s=1}^{n} a_{i j}\|f(i, s)-h(j, s)\|_{X}^{p} \\
&+\frac{2^{p-1} \gamma_{+}\left(B,\|\cdot\|_{X}^{p}\right)}{m n} \sum_{s=1}^{n} \sum_{t=1}^{n} \sum_{j=1}^{m} b_{s t}\|h(j, s)-g(j, t)\|_{X}^{p} \\
& \stackrel{(247)}{\leqslant} \frac{\Gamma}{m n} \sum_{i=1}^{m} \sum_{j=1}^{n} a_{i j} \sum_{s=1}^{n}\left(\|f(i, s)-h(j, s)\|_{X}^{p}+c \sum_{t=1}^{n} b_{s t}\|h(j, s)-g(j, t)\|_{X}^{p}\right) \\
& \frac{\Gamma}{m n} \sum_{i=1}^{m} \sum_{j=1}^{n} \sum_{s=1}^{n} \sum_{t=1}^{n} a_{i j} b_{s t}\|f(i, s)-g(j, t)\|_{X}^{p} \cdot
\end{aligned}
$$

Since (253) holds for every $f, g:\{1, \ldots, m\} \times\{1, \ldots, n\} \rightarrow X$, (246) follows.

8.2. Sub-multiplicativity for the zigzag product. Here we prove Theorem 1.3. Before doing so, we need to recall the definition of the zigzag product of Reingold, Vadhan and Wigderson [64]. The notation used below, which lends itself well to the ensuing proof of Theorem 1.3, was suggested to us by K. Ball.

Fix $n_{1}, d_{1}, d_{2} \in \mathbb{N}$. Suppose that $G_{1}=\left(V_{1}, E_{1}\right)$ is an $n_{1}$-vertex graph which is $d_{1}$-regular and that $G_{2}=\left(V_{2}, E_{2}\right)$ is a $d_{1}$-vertex graph which is $d_{2}$-regular. Since the number of vertices in $G_{2}$ is the same as the degree of $G_{1}$, we can identify $V_{2}$ with the edges emanating from a given vertex $u \in V_{1}$. Formally, we fix for every $u \in V_{1}$ a bijection

$$
\pi_{u}:\left\{(u, v) \in\{u\} \times V_{1}:(u, v) \in E_{1}\right\} \rightarrow V_{2} .
$$

Moreover, we fix for every $a \in V_{2}$ a bijection between $\left\{1, \ldots, d_{2}\right\}$ and the multiset of the vertices adjacent to $a$ in $G_{2}$, i.e.,

$$
\kappa_{a}:\left\{1, \ldots, d_{2}\right\} \rightarrow\left\{b \in V_{2}:(a, b) \in E_{2}\right\} .
$$

The zigzag product $G_{1}$ (2) $G_{2}$ is the graph whose vertices are $V_{1} \times V_{2}$ and the ordered pair $((u, a),(v, b)) \in V_{1} \times V_{2}$ is added to $E\left(G_{1}(2) G_{2}\right)$ whenever there exist $i, j \in\left\{1, \ldots, d_{2}\right\}$ satisfying

Thus,

$$
(u, v) \in E_{1} \quad \text { and } \quad a=\kappa_{\pi_{u}(u, v)}(i) \quad \text { and } \quad b=\kappa_{\pi_{v}(v, u)}(j)
$$

$$
E\left(G_{1}(2) G_{2}\right)((u, a),(v, b)) \stackrel{\text { def }}{=} \sum_{i=1}^{d_{2}} \sum_{j=1}^{d_{2}} E_{1}(u, v) \cdot \mathbf{1}_{\left\{a=\kappa_{\pi_{u}(u, v)}(i)\right\}} \cdot \mathbf{1}_{\left\{b=\kappa_{\pi_{v}(u, v)}(j)\right\}} \cdot
$$

The schematic description of this construction is as follows. Think of the vertex set of $G_{1}$ (2) $G_{2}$ as a disjoint union of "clouds" which are copies of $V_{2}=\left\{1, \ldots, d_{1}\right\}$ indexed by $V_{1}$. Thus $(u, a)$ is the point indexed by $a$ in the cloud labeled by $u$. Every edge $((u, a),(v, b))$ of $G_{1}(2) G_{2}$ is the result of a three step walk: a "zig" step in $G_{2}$ from $a$ to $\pi_{u}(u, v)$ in $u$ 's cloud, a "zag" step in $G_{1}$ from $u$ 's cloud to $v$ 's cloud along the edge $(u, v)$ and a final "zig" step in $G_{2}$ from $\pi_{v}(u, v)$ to $b$ in $v$ 's cloud. The zigzag product is illustrated in Figure 2. The 
number of vertices of $G_{1}$ (2) $G_{2}$ is $n_{1} d_{1}$ and its degree is $d_{2}^{2}$. The zigzag product depends on

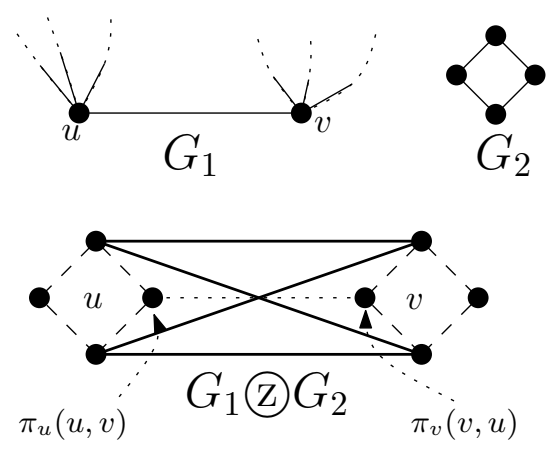

FIgURE 2. A schematic illustration of the zigzag product. The upper part of the figure depicts part of a 4 -regular graph $G_{1}$, and a 4 -vertex cycle $G_{2}$. The bottom part of the figure depicts the edges of the zigzag product between $u$ 's cloud and $v$ 's cloud. The original edges of $G_{1}$ and $G_{2}$ are drawn as dotted and dashed lines, respectively.

the choice of labels $\left\{\pi_{u}\right\}_{u \in V_{1}}$, and in fact different labels of the same graphs can produce non-isomorphic products 3 . However, the estimates below will be independent of the actual choice of the labeling, so while our notation should formally depend on the labeling, we will drop its explicit mention for the sake of simplicity.

Proof of Theorem 1.3. Fix $f, g: V_{1} \times V_{2} \rightarrow X$. The definition of $\gamma_{+}\left(G_{1}, K\right)$ implies that for all $a, b \in V_{2}$ we have

$$
\frac{1}{n_{1}^{2}} \sum_{(u, v) \in V_{1} \times V_{1}} K(f(u, a), g(v, b)) \leqslant \frac{\gamma_{+}\left(G_{1}, K\right)}{n_{1} d_{1}} \sum_{(u, v) \in E_{1}} K(f(u, a), g(v, b)) .
$$

Hence,

$$
\begin{aligned}
& \frac{1}{\left|V_{1} \times V_{2}\right|^{2}} \sum_{((u, a),(v, b)) \in\left(V_{1} \times V_{2}\right) \times\left(V_{1} \times V_{2}\right)} K(f(u, a), g(v, b)) \\
& =\frac{1}{d_{1}^{2}} \sum_{(a, b) \in V_{2} \times V_{2}} \frac{1}{n_{1}^{2}} \sum_{(u, v) \in V_{1} \times V_{1}} K(f(u, a), g(v, b)) \\
& \stackrel{\text { (257) }}{\leqslant} \frac{\gamma_{+}\left(G_{1}, K\right)}{n_{1} d_{1}^{3}} \sum_{(a, b) \in V_{2} \times V_{2}} \sum_{(u, v) \in E_{1}} K(f(u, a), g(v, b)) \text {. }
\end{aligned}
$$

Next, fix $u \in V_{1}$ and $b \in V_{2}$, and define $\phi_{b}^{u}: V_{2} \rightarrow X$ as follows. Recalling (254), for $c \in V_{2}$ write $\pi_{u}^{-1}(c)=(u, v) \in E_{1}$ for some $v \in V_{1}$, and define $\phi_{b}^{u}(c)=g(v, b)$. The definition of

\footnotetext{
${ }^{3}$ The labels $\left\{\kappa_{a}\right\}_{a \in V_{2}}$ do not affect the structure of the zigzag product but they are useful in the subsequent analysis.
} 
$\gamma_{+}\left(G_{2}, K\right)$ implies that

$$
\begin{array}{r}
\frac{1}{d_{1}^{2}} \sum_{a \in V_{2}} \sum_{\substack{v \in V_{1} \\
(u, v) \in E_{1}}} K(f(u, a), g(v, b))=\frac{1}{d_{1}^{2}} \sum_{a \in V_{2}} \sum_{c \in V_{2}} K\left(f(u, a), \phi_{b}^{u}(c)\right) \\
\leqslant \frac{\gamma_{+}\left(G_{2}, K\right)}{d_{1} d_{2}} \sum_{\substack{v \in V_{1} \\
(u, v) \in E_{1}}} \sum_{i=1}^{d_{2}} K\left(f\left(u, \kappa_{\pi_{u}(u, v)}(i)\right), g(v, b)\right),
\end{array}
$$

Summing (259) over $u \in V_{1}$ and $b \in V_{2}$ and substituting the resulting expression into (258) yields the bound

$$
\begin{aligned}
& \frac{1}{\left|V_{1} \times V_{2}\right|^{2}} \sum_{((u, a),(v, b)) \in\left(V_{1} \times V_{2}\right) \times\left(V_{1} \times V_{2}\right)} K(f(u, a), g(v, b)) \\
& \leqslant \frac{\gamma_{+}\left(G_{1}, K\right) \gamma_{+}\left(G_{2}, K\right)}{n_{1} d_{1}^{2} d_{2}} \sum_{v \in V_{1}} \sum_{i=1}^{d_{2}} \sum_{\substack{u \in V_{1} \\
(u, v) \in E_{1}}} \sum_{b \in V_{2}} K\left(f\left(u, \kappa_{\pi_{u}(u, v)}(i)\right), g(v, b)\right) .
\end{aligned}
$$

Fix $i \in\left\{1, \ldots, d_{2}\right\}$ and $v \in V_{1}$, and define $\psi_{i}^{v}: V_{2} \rightarrow X$ as follows. For $c \in V_{2}$ write $\pi_{v}^{-1}(c)=(v, u)$ for some $u \in V_{1}$ such that $(v, u) \in E_{1}$ (equivalently, $(u, v) \in E_{1}$ ), and set $\psi_{i}^{v}(c)=f\left(u, \kappa_{\pi_{u}(u, v)}(i)\right)$. Another application of the definition of $\gamma_{+}\left(G_{2}, K\right)$ implies that

$$
\begin{array}{r}
\frac{1}{d_{1}^{2}} \sum_{\substack{u \in V_{1} \\
(u, v) \in E_{1}}} \sum_{b \in V_{2}} K\left(f\left(u, \kappa_{\pi_{u}(u, v)}(i)\right), g(v, b)\right)=\frac{1}{d_{1}^{2}} \sum_{c \in V_{2}} \sum_{b \in V_{2}} K\left(\psi_{i}^{v}(c), g(v, b)\right) \\
\leqslant \frac{\gamma_{+}\left(G_{2}, K\right)}{d_{1} d_{2}} \sum_{\substack{u \in V_{1} \\
(u, v) \in E_{1}}} \sum_{j=1}^{d_{2}} K\left(f\left(u, \kappa_{\pi_{u}(u, v)}(i)\right), g\left(v, \kappa_{\pi_{v}(v, u)}(j)\right)\right) .
\end{array}
$$

Summing (261) over $v \in V_{1}$ and $i \in\left\{1, \ldots, d_{2}\right\}$, and combining the resulting inequality with (260), yields the bound

$$
\begin{aligned}
& \frac{1}{\left|V_{1} \times V_{2}\right|^{2}} \sum_{((u, a),(v, b)) \in\left(V_{1} \times V_{2}\right) \times\left(V_{1} \times V_{2}\right)} K(f(u, a), g(v, b)) \\
& \leqslant \quad \frac{\gamma_{+}\left(G_{1}, K\right) \gamma_{+}\left(G_{2}, K\right)^{2}}{n_{1} d_{1} d_{2}^{2}} \sum_{(u, v) \in E_{1}} \sum_{i=1}^{d_{2}} \sum_{j=1}^{d_{2}} K\left(f\left(u, \kappa_{\pi_{u}(u, v)}(i)\right), g\left(v, \kappa_{\pi_{v}(v, u)}(j)\right)\right) \\
& \stackrel{(256)}{=} \quad \frac{\gamma_{+}\left(G_{1}, K\right) \gamma_{+}\left(G_{2}, K\right)^{2}}{n_{1} d_{1} d_{2}^{2}} \sum_{((u, a),(v, b)) E\left(G_{1}(2) G_{2}\right)} K(f(u, a), g(v, b)) .
\end{aligned}
$$

Since (262) holds for every $f, g: V_{1} \times V_{2} \rightarrow X$, the proof of Theorem 1.3 is complete.

8.3. Sub-multiplicativity for replacement products. Here we continue to use the notation of Section 8.2. Specifically, we fix $n_{1}, d_{1}, d_{2} \in \mathbb{N}$ and suppose that $G_{1}=\left(V_{1}, E_{1}\right)$ is an $n_{1}$-vertex graph which is $d_{1}$-regular and that $G_{2}=\left(V_{2}, E_{2}\right)$ is a $d_{1}$-vertex graph which is $d_{2}$-regular. We also identify $V_{1}=\left\{1, \ldots, n_{1}\right\}$ and $V_{2}=\left\{1, \ldots, d_{1}\right\}$, and for every $u \in V_{1}$ 
and $a \in V_{2}$ we fix a bijections $\pi_{u}$ and $\kappa_{a}$ as in (254) and (255), respectively. The replacement product [17, 64] of $G_{1}$ and $G_{2}$, denoted $G_{1} \odot G_{2}$, is the graph with vertex set $\left\{1, \ldots, n_{1}\right\} \times\left\{1, \ldots, d_{1}\right\}$ in which the ordered pair $((u, i),(v, j)) \in\left\{1, \ldots, n_{1}\right\} \times\left\{1, \ldots, d_{1}\right\}$ is added to $E\left(G_{1} \odot G_{2}\right)$ if and only if either $u=v$ and $(i, j) \in E_{2}$ or $(u, v) \in E_{1}$ and $i=\pi_{u}(u, v)$ and $j=\pi_{v}(v, u)$. Thus,

$$
E\left(G_{1} \mathrm{P} G_{2}\right)((u, i),(v, j)) \stackrel{\text { def }}{=} E_{2}(i, j) \cdot \mathbf{1}_{\{u=v\}}+E_{1}(u, v) \cdot \mathbf{1}_{\left\{i=\pi_{u}(u, v)\right\}} \cdot \mathbf{1}_{\left\{j=\pi_{v}(v, u)\right\}} \cdot
$$

This definition makes $G_{1}$ P $G_{2}$ be a $\left(d_{2}+1\right)$-regular graph.

The following lemma shows that the "discrete gradient" associated to $G_{1}(2) G_{2}$ is dominated by $3^{p-1}\left(d_{2}+1\right)$ times the "discrete gradient" associated to $\left.G_{1} \mathrm{P}\right) G_{2}$.

Lemma 8.3. Fix $p \in[1, \infty)$, a metric space $\left(X, d_{X}\right)$ and $n_{1}, d_{1}, d_{2} \in \mathbb{N}$. Suppose that $G_{1}=\left(V_{1}, E_{1}\right)$ is an $n_{1}$-vertex graph which is $d_{1}$-regular and that $G_{2}=\left(V_{2}, E_{2}\right)$ is a d $d_{1}$-vertex graph which is $d_{2}$-regular. Then every $f, g: V_{1} \times V_{2} \rightarrow X$ satisfy

$$
\begin{aligned}
& \frac{1}{\left|E\left(G_{1}(2) G_{2}\right)\right|} \sum_{((u, a),(v, b)) \in E\left(G_{1}(2) G_{2}\right)} d_{X}(f(u, a), g(v, b))^{p} \\
& \leqslant \frac{3^{p-1}\left(d_{2}+1\right)}{\left|E\left(G_{1} \mathrm{\Gamma} G_{2}\right)\right|} \sum_{((u, a),(v, b)) \in E\left(G_{1}\left(G_{2}\right)\right.} d_{X}(f(u, a), g(v, b))^{p} .
\end{aligned}
$$

Before proving Lemma 8.3 we record two of its immediate (yet useful) consequences.

Corollary 8.4. Under the assumptions of Lemma 8.3 we have

$$
\left.\gamma_{+}\left(G_{1} \mathrm{P}\right) G_{2}, d_{X}^{p}\right) \leqslant 3^{p-1}\left(d_{2}+1\right) \cdot \gamma_{+}\left(G_{1}(\mathrm{Z}) G_{2}, d_{X}^{p}\right)
$$

Now, part (IV) of Theorem 1.13 corresponds to the case $p=2$ of the following combination of Theorem 1.3 and Corollary 8.4 .

Corollary 8.5. Under the assumptions of Lemma 8.3 we have

$$
\gamma_{+}\left(G_{1} \mathrm{P} G_{2}, d_{X}^{p}\right) \leqslant 3^{p-1}\left(d_{2}+1\right) \cdot \gamma_{+}\left(G_{1}, d_{X}^{p}\right) \cdot \gamma_{+}\left(G_{2}, d_{X}^{p}\right)^{2}
$$

Proof of Lemma 8.3. Fix $((u, a),(v, b)) \in E\left(G_{1}\right.$ (Z) $\left.G_{2}\right)$. Thus by the definition of the zigzag product we have $(u, v) \in E_{1}$ and $\left(a, \pi_{u}(u, v)\right),\left(b, \pi_{v}(v, u)\right) \in E_{2}$. Observe that the following three pairs are edges of $G_{1} \Upsilon G_{2}$.

$$
\left((u, a),\left(u, \pi_{u}(u, v)\right),\left(\left(u, \pi_{u}(u, v),\left(v, \pi_{v}(v, u)\right),\left(\left(v, \pi_{v}(v, u),(v, b)\right) .\right.\right.\right.\right.
$$

By the triangle inequality,

$$
\begin{aligned}
& d_{X}(f(u, a), g(v, b))^{p} \leqslant 3^{p-1}\left(d_{X}\left(f(u, a), g\left(u, \pi_{u}(u, v)\right)\right)^{p}\right. \\
& \left.\left.\quad+d_{X}\left(g\left(u, \pi_{u}(u, v)\right), f\left(v, \pi_{v}(v, u)\right)\right)^{p}+d_{X}\left(f\left(v, \pi_{v}(v, u)\right), g(v, b)\right)\right)^{p}\right) .
\end{aligned}
$$


Therefore,

$$
\begin{aligned}
& \frac{1}{\left|E\left(G_{1}(\mathrm{Z}) G_{2}\right)\right|} \sum_{((u, a),(v, b)) \in E\left(G_{1}(2) G_{2}\right)} d_{X}(f(u, a), g(v, b))^{p} \\
& =\frac{1}{n_{1} d_{1} d_{2}^{2}} \sum_{\substack{(u, v) \in E_{1} \\
\left(a, \pi_{u}(u, v)\right) \in E_{2}}} \sum_{\substack{a \in V_{2} \\
\left(b, \pi_{v}(v, u)\right) \in V_{2}}} d_{X}(f(u, a), g(v, b))^{p} \\
& \stackrel{\frac{\sqrt{264})}{\leqslant}}{\frac{3^{p-1}}{n_{1} d_{1} d_{2}^{2}}}\left(S_{1}+S_{2}+S_{3}\right),
\end{aligned}
$$

where the quantities $S_{1}, S_{2}, S_{3}$ are defined as follows.

$$
\begin{aligned}
& S_{1} \stackrel{\text { def }}{=} \sum_{(u, v) \in E_{1}} \sum_{\substack{a \in V_{2} \\
\left(a, \pi_{u}(u, v)\right) \in E_{2}}} \sum_{\substack{b \in V_{2} \\
\left(b, \pi_{v}(v, u)\right) \in E_{2}}} d_{X}\left(f(u, a), g\left(u, \pi_{u}(u, v)\right)\right)^{p} \\
& =d_{2} \sum_{(u, v) \in E_{1}} \sum_{\substack{a \in V_{2} \\
\left(a, \pi_{u}(u, v)\right) \in E_{2}}} d_{X}\left(f(u, a), g\left(u, \pi_{u}(u, v)\right)\right)^{p}, \\
& S_{2} \stackrel{\text { def }}{=} \sum_{(u, v) \in E_{1}} \sum_{\substack{a \in V_{2} \\
\left(a, \pi_{u}(u, v)\right) \in E_{2}}} \sum_{\substack{b \in V_{2} \\
\left(b, \pi_{v}(v, u)\right) \in E_{2}}} d_{X}\left(g\left(u, \pi_{u}(u, v)\right), f\left(v, \pi_{v}(v, u)\right)\right)^{p} \\
& =d_{2}^{2} \sum_{(u, v) \in E_{1}} d_{X}\left(g\left(u, \pi_{u}(u, v)\right), f\left(v, \pi_{v}(v, u)\right)\right)^{p}, \\
& \left.S_{3} \stackrel{\text { def }}{=} \sum_{(u, v) \in E_{1}} \sum_{\substack{a \in V_{2} \\
\left(a, \pi_{u}(u, v)\right) \in E_{2}}} \sum_{\substack{b \in V_{2} \\
\left(b, \pi_{v}(v, u)\right) \in E_{2}}} d_{X}\left(f\left(v, \pi_{v}(v, u)\right), g(v, b)\right)\right)^{p} \\
& \left.=d_{2} \sum_{(u, v) \in E_{1}} \sum_{\substack{\left.b \in V_{2} \\
\left(b, \pi_{v}(v, u)\right)\right\} \in E_{2}}} d_{X}\left(f\left(v, \pi_{v}(v, u)\right), g(v, b)\right)\right)^{p} .
\end{aligned}
$$

By the definition of the replacement product we have

$$
\begin{aligned}
S_{1}+ & S_{2}+S_{3} \\
& =d_{2} \sum_{u \in V_{1}} \sum_{(i, j) \in E_{2}} d_{X}(f(u, i), g(u, j))^{p}+d_{2}^{2} \sum_{(u, v) \in E_{1}} d_{X}\left(g\left(u, \pi_{u}(u, v)\right), f\left(v, \pi_{v}(v, u)\right)\right)^{p} \\
& \leqslant d_{2}^{2} \sum_{((u, i),(v, j)) \in E\left(G_{1}(\mathrm{r}) G_{2}\right)} d_{X}(f(u, i), g(v, j))^{p} .
\end{aligned}
$$

Recalling that $\left|E\left(G_{1} \odot G_{2}\right)\right|=n_{1} d_{1}\left(d_{2}+1\right)$, the desired estimate (263) is now a consequence of (265) and (266).

The balanced replacement product of $G_{1}$ and $G_{2}$, denoted $G_{1}$ (b) $G_{2}$, is a useful variant of $G_{1} \mathrm{P} G_{2}$ that was introduced in [64]. The vertex set of $G_{1}$ (b) $G_{2}$ is still $\left\{1, \ldots, n_{1}\right\} \times\left\{1, \ldots, d_{1}\right\}$, 
but the edges of $G_{1}$ (b) $G_{2}$ are now given by

$$
\begin{aligned}
& \forall((u, i),(v, j)) \in\left\{1, \ldots, n_{1}\right\} \times\left\{1, \ldots, d_{1}\right\}, \\
& \quad E\left(G_{1} \text { (b) } G_{2}\right)((u, i),(v, j)) \stackrel{\text { def }}{=} E_{2}(i, j) \cdot \mathbf{1}_{\{u=v\}}+d_{2} E_{1}(u, v) \cdot \mathbf{1}_{\left\{i=\pi_{u}(u, v)\right\}} \cdot \mathbf{1}_{\left\{j=\pi_{v}(v, u)\right\}} .
\end{aligned}
$$

This definition makes $G_{1}$ (b) $G_{2}$ be a $2 d_{2}$-regular graph.

Arguing analogously to the proof of Lemma 8.3, we have the following statements.

Lemma 8.6. Fix $p \in[1, \infty)$, a metric space $\left(X, d_{X}\right)$ and $n_{1}, d_{1}, d_{2} \in \mathbb{N}$. Suppose that $G_{1}=\left(V_{1}, E_{1}\right)$ is an $n_{1}$-vertex graph which is $d_{1}$-regular and that $G_{2}=\left(V_{2}, E_{2}\right)$ is a d $d_{1}$-vertex graph which is $d_{2}$-regular. Then every $f, g: V_{1} \times V_{2} \rightarrow X$ satisfy

$$
\begin{aligned}
& \frac{1}{\mid E\left(G_{1} \text { (2) } G_{2}\right) \mid} \sum_{((u, a),(v, b)) \in E\left(G_{1} \text { (2) } G_{2}\right)} d_{X}(f(u, a), g(v, b))^{p} \\
& \leqslant \frac{2 \cdot 3^{p-1}}{\mid E\left(G_{1} \text { (b) } G_{2}\right) \mid} \sum_{((u, a),(v, b)) \in E\left(G_{1} \text { (b) } G_{2}\right)} d_{X}(f(u, a), g(v, b))^{p} .
\end{aligned}
$$

Corollary 8.7. Under the assumptions of Lemma 8.6 we have

$$
\gamma_{+}\left(G_{1}(\mathrm{~b}) G_{2}, d_{X}^{p}\right) \leqslant 2 \cdot 3^{p-1} \cdot \gamma_{+}\left(G_{1}\left(\mathrm{Z} G_{2}, d_{X}^{p}\right) .\right.
$$

Part (V) of Theorem 1.13 corresponds to the case $p=2$ of the following combination of Theorem 1.3 and Corollary 8.7 .

Corollary 8.8. Under the assumptions of Lemma 8.6 we have

$$
\gamma_{+}\left(G_{1} \text { (b) } G_{2}, d_{X}^{p}\right) \leqslant 2 \cdot 3^{p-1} \cdot \gamma_{+}\left(G_{1}, d_{X}^{p}\right) \cdot \gamma_{+}\left(G_{2}, d_{X}^{p}\right)^{2}
$$

Remark 8.9. An analysis of the behavior of spectral gaps under the balanced replacement product was previously performed in a non-Euclidean setting by Alon, Schwartz and Shapira [1]. Specifically, [1, Thm. 1.3] estimates the edge expansion of $G_{1}$ (b) $G_{2}$ in terms of the edge expansion of $G_{1}$ and $G_{2}$ via a direct combinatorial argument. The edge expansion of a graph $G$ is equivalent up to universal constant factors to $\gamma(G,|\cdot|)$, where $|\cdot|$ is the standard absolute value on $\mathbb{R}$. The corresponding bound arising from Corollary 8.8 is better than the bound of [1, Thm. 1.3] in terms of constant factors.

8.4. Sub-multiplicativity for derandomized squaring. Here we continue to use the notation of Section 8.2 and Section 8.3. The derandomized squaring of $G_{1}$ and $G_{2}$, as introduced by Rozenman and Vadhan in [66] and denoted $G_{1}\left(\mathrm{~S} G_{2}\right.$, is defined as follows. The vertex set of $G_{1} \mathrm{~S} G_{2}$ is $V_{1}=\left\{1, \ldots, n_{1}\right\}$, and the edges $E\left(G_{1} \mathrm{~S} G_{2}\right)$ are given by

$$
\forall(u, v) \in V_{1} \times V_{1}, \quad E\left(G_{1} \mathrm{~S} G_{2}\right)(u, v) \stackrel{\text { def }}{=} \sum_{w \in V_{1}} E_{1}(w, u) E_{1}(w, v) E_{2}\left(\pi_{w}(w, u), \pi_{w}(w, v)\right) .
$$

Thus, given $(u, v) \in V_{1} \times V_{1}$, we add a copy of $(u, v)$ to $E\left(G_{1}\left(G_{2}\right)\right.$ for every $(i, j) \in E_{2}$ such that there exists $w \in V_{1}$ with $(w, u),(w, v) \in E_{1}$ and $\pi_{w}(w, u)=i, \pi_{w}(w, v)=j$. With this definition one checks that $G_{1} \subseteq G_{2}$ is $d_{1} d_{2}$-regular.

The following proposition corresponds to part (III) of Theorem 1.13. 
Proposition 8.10. Fix $n_{1}, d_{1}, d_{2} \in \mathbb{N}$ and suppose that $G_{1}=\left(V_{1}, E_{1}\right)$ is an $n_{1}$-vertex graph which is $d_{1}$-regular and that $G_{2}=\left(V_{2}, E_{2}\right)$ is a $d_{1}$-vertex graph which is $d_{2}$-regular. Then for every kernel $K: X \times X \rightarrow[0, \infty)$ we have

$$
\gamma_{+}\left(G_{1} \mathrm{~S} G_{2}, K\right) \leqslant \gamma_{+}\left(G_{1}^{2}, K\right) \gamma_{+}\left(G_{2}, K\right) .
$$

In [66] Rozenman and Vadhan used a spectral argument to prove the Euclidean case of (268), i.e., the special case of (268) when $K: \mathbb{R} \times \mathbb{R} \rightarrow[0, \infty)$ is given by $K(x, y)=(x-y)^{2}$.

Proof of Proposition 8.10. Fix $f, g: V_{1} \rightarrow X$. The definition of $\gamma_{+}\left(G_{1}^{2}, K\right)$ implies that

$$
\begin{aligned}
\frac{1}{n_{1}^{2}} \sum_{(u, v) \in V_{1} \times V_{1}} K(f(u), f(v)) \leqslant \frac{\gamma_{+}\left(G_{1}^{2}, K\right)}{n_{1} d_{1}^{2}} \sum_{(u, v) \in E\left(G_{1}^{2}\right)} K(f(u), f(v)) \\
=\frac{\gamma_{+}\left(G_{1}^{2}, K\right)}{n_{1} d_{1}^{2}} \sum_{w \in V_{1}} \sum_{(u, w) \in E_{1}} \sum_{(w, v) \in E_{1}} K(f(u), g(v)) .
\end{aligned}
$$

For every fixed $w \in V_{1}$ define $\phi^{w}, \psi^{w}: V_{2} \rightarrow X$ as follows. For $i, j \in V_{2}$ consider the unique vertices $u, v \in V_{1}$ such that $\pi_{w}(w, u)=i$ and $\pi_{w}(w, v)=j$, and define $\phi^{w}(i)=f(u)$ and $\psi^{w}(j)=g(v)$. The definition of $\gamma_{+}\left(G_{2}, K\right)$ implies that

$$
\begin{aligned}
& \frac{1}{d_{1}^{2}} \sum_{(u, w) \in E_{1}} \sum_{(w, v) \in E_{1}} K(f(u), g(v))=\frac{1}{d_{1}^{2}} \sum_{(i, j) \in V_{2} \times V_{2}} K\left(\phi^{w}(i), \psi^{w}(j)\right) \\
& \quad \leqslant \frac{\gamma_{+}\left(G_{2}, K\right)}{d_{1} d_{2}} \sum_{(i, j) \in E_{2}} K\left(\phi^{w}(i), \psi^{w}(j)\right) \\
& \quad=\frac{\gamma_{+}\left(G_{2}, K\right)}{d_{1} d_{2}} \sum_{(u, w) \in E_{1}} \sum_{(w, v) \in E_{1}} E_{2}\left(\pi_{w}(w, u), \pi_{w}(w, v)\right) K(f(u), g(v)) .
\end{aligned}
$$

The definition of $G_{1} \mathrm{~S} G_{2}$ in combination with (269) and (270) now yields the estimate

$$
\begin{aligned}
& \frac{1}{n_{1}^{2}} \sum_{(u, v) \in V_{1} \times V_{1}} K(f(u), f(v)) \\
& \leqslant \frac{\gamma_{+}\left(G_{1}^{2}, K\right) \gamma_{+}\left(G_{2}, K\right)}{n_{1} d_{1} d_{2}} \sum_{w \in V_{1}} \sum_{(u, w) \in E_{1}} \sum_{(w, v) \in E_{1}} E_{2}\left(\pi_{w}(w, u), \pi_{w}(w, v)\right) K(f(u), g(v)) \\
& =\frac{\gamma_{+}\left(G_{1}^{2}, K\right) \gamma_{+}\left(G_{2}, K\right)}{n_{1} d_{1} d_{2}} \sum_{(x, y) \in E\left(G_{1}\left(G_{2}\right)\right.} K(f(x), g(y)) .
\end{aligned}
$$

\section{Counterexamples}

9.1. Expander families need not embed coarsely into each other. As was mentioned in the introduction, it is an open question whether every classical (i.e., Euclidean) expander graph family is also a super-expander. Here we rule out the most obvious approach towards such a result: to embed coarsely any expander family in any other expander family. Formally, given two families of metric spaces $\mathscr{X}, \mathscr{Y}$, we say that $\mathscr{X}$ admits a coarse embedding into 
$\mathscr{Y}$ if there exist non-decreasing $\alpha, \beta:[0, \infty) \rightarrow[0, \infty)$ satisfying $\lim _{t \rightarrow \infty} \alpha(t)=\infty$ such that for every $\left(X, d_{X}\right) \in \mathscr{X}$ there exists $\left(Y, d_{Y}\right) \in \mathscr{Y}$ and a mapping $f: X \rightarrow Y$ that satisfies

$$
\forall x, y \in X, \quad \alpha\left(d_{X}(x, y)\right) \leqslant d_{Y}(f(x), f(y)) \leqslant \beta\left(d_{X}(x, y)\right) .
$$

This condition clearly implies that $\alpha(0)=0$, and for notational convenience we also assume without loss of generality that $\beta(0)=0$.

Let $\mathscr{C}$ denote the set of all increasing sub-additive functions $\omega:[0, \infty) \rightarrow[0, \infty)$ with $\omega(0)=0$. If $\left(X, d_{X}\right)$ is a metric space and $\omega \in \mathscr{C}$ then $\left(X, \omega \circ d_{X}\right)$ is also a metric space, known as the metric transform of $\left(X, d_{X}\right)$ by $\omega$.

In what follows, given a connected graph $G=(V, E)$, the geodesic metric induced by $G$ on $V$ will be denoted $d_{G}$. Recall that a sequence of graphs $\left\{G_{n}\right\}_{n=1}^{\infty}$ is called a constant degree expander sequence if there exists $d \in \mathbb{N}$ such that each $G_{n}$ is $d$-regular and $\sup _{n \in \mathbb{N}} \lambda\left(G_{n}\right)<1$. The purpose of this section is to prove the following result.

Theorem 9.1. There exist two constant degree expander sequences $\left\{G_{i}\right\}_{i=1}^{\infty}$ and $\left\{H_{i}\right\}_{i=1}^{\infty}$ such that $\left\{\left(V\left(H_{i}\right), d_{H_{i}}\right)\right\}_{i=1}^{\infty}$ does not admit a coarse embedding into the family of metric spaces $\left\{\left(V\left(G_{i}\right), \omega \circ d_{G_{i}}\right):(i, \omega) \in \mathbb{N} \times \mathscr{C}\right\}$.

Proof. It is well known (see e.g. [36, 38) that there exists $c \in(0, \infty)$, an integer $d \geqslant 3$, and a sequence of $d$-regular expanders $\left\{G_{i}\right\}_{i=1}^{\infty}$ such that if we set $n_{i}=\left|V\left(G_{i}\right)\right|$ then $\left\{n_{i}\right\}_{i=1}^{\infty}$ is strictly increasing and each $G_{i}$ has girth at least $4 c \log n_{i}$. By adjusting $c$ to be a smaller constant if necessary (as we may), we assume below that

$$
c \log n_{i}<\frac{n_{i}}{2(d+1)^{2 c \log n_{i}}} .
$$

We also assume throughout the ensuing argument that $c \log n_{i}>7$ for all $i \in \mathbb{N}$.

The desired expander sequence $\left\{H_{i}\right\}_{i=1}^{\infty}$ will be constructed by modifying $\left\{G_{i}\right\}_{i=1}^{\infty}$ so as to contain sufficiently many short cycles. Specifically, fix $i \in \mathbb{N}$ and write $G_{i}=\left(V_{i}, E_{i}\right)$. We will construct $H_{i}=\left(V_{i}, F_{i}\right)$ with $F_{i} \supsetneq E_{i}$, i.e., $H_{i}$ will be a graph with the same vertices as $G_{i}$ but with additional edges. The construction will ensure that

$$
\operatorname{diam}\left(H_{i}\right) \geqslant \frac{c}{2} \log n_{i}
$$

(Here, and in what follows, diameters of graphs are always understood to be with respect to their shortest-path metric.) We will also ensure that for every integer $h \in\left[3, c \log n_{i}\right]$ the graph $H_{i}$ contains a cycle of length $h$ which is embedded isometrically into $\left(H_{i}, d_{H_{i}}\right)$, i.e., there exist $x_{1}, \ldots, x_{h} \in V_{i}$ such that $d_{H_{i}}\left(x_{a}, x_{b}\right)=\min \{|a-b|, h-|a-b|\}$ for every $a, b \in\{1, \ldots, h\}$, and $\left\{x_{1}, x_{2}\right\},\left\{x_{2}, x_{3}\right\}, \ldots,\left\{x_{h-1}, x_{h}\right\},\left\{x_{h}, x_{1}\right\} \in F_{i}$.

Set

$$
\ell \stackrel{\text { def }}{=}\left\lfloor c \log n_{i}\right\rfloor \text {. }
$$

We will define inductively sets of edges $E=F^{0} \subsetneq F^{1} \subsetneq \ldots \subsetneq F^{\ell}$ with $\left|F_{j} \backslash F_{j-1}\right|=1$ for all $j \in\{1, \ldots, \ell\}$. Fix $j \in\{0, \ldots, \ell-1\}$ and assume inductively that $F^{j}$ has already been defined so that the graph

$$
G_{i}^{j} \stackrel{\text { def }}{=}\left(V_{i}, F^{j}\right)
$$

has maximal degree at most $d+1$. Write

$$
M_{j} \stackrel{\text { def }}{=}\left\{u \in V_{i}: \exists e \in F^{j} \backslash E, u \in e\right\}=\bigcup_{e \in F^{j} \backslash E} e .
$$


Thus $\left|M_{j}\right| \leqslant 2 j$. Hence, if we set

$$
D_{j} \stackrel{\text { def }}{=}\left\{u \in V_{i}: d_{G_{i}^{j}}\left(u, M_{j}\right) \leqslant 2 c \log n_{i}\right\}
$$

then

$$
\left|D_{j}\right| \leqslant 2 j(d+1)^{2 c \log n_{i}} \leqslant 2 \ell(d+1)^{2 c \log n_{i}} \stackrel{(271) \wedge(273)}{<} n_{i} .
$$

Therefore $V \backslash D_{j} \neq \emptyset$. Choose an arbitrary vertex $x \in V \backslash D_{j}$. Since $G_{i}$ has girth at least $4 c \log n_{i}$ and $j \leqslant \ell$, there exists $y \in V$ with $d_{G_{i}}(x, y)=j+2$. Define $F^{j+1}=F^{j} \cup\{\{x, y\}\}$. This creates a new cycle of length $j+3$.

By construction, the graph $G_{i}^{j+1} \stackrel{\text { def }}{=}\left(V_{i}, F^{j+1}\right)$ contains a cycle $C_{h}$ of length $h$ for every $h \in\{3, \ldots, j+3\}$. Moreover, we claim that these cycles are embedded isometrically into the metric space $\left(V_{i}, d_{G_{i}^{j+1}}\right)$. Indeed, due to the choice of $x$, if $h \in\{3, \ldots, j+2\}$ then

$$
d_{G_{i}^{j}}\left(C_{h},\{x, y\}\right)>2 c \log n_{i}-(j+2),
$$

which is at least $h / 2$ (the diameter of $C_{h}$ ) because $c \log n_{i}>7$. Thus the new edge $\{x, y\}$ does not change the isometric embeddability of $C_{h}$. The new cycle $C_{j+3}$ is isometrically embedded into $\left(V_{i}, d_{G_{i}}\right)$ since the girth of $G_{i}$ is at least $4 c \log n_{i}>2(j+2)$. Since

$$
d_{G_{i}^{j}}\left(M_{j}, C_{j+3}\right)>2 c \log n_{i}-(j+2)>\frac{j+3}{2},
$$

The cycle $C_{j+3}$ remains isometrically embedded into $\left(V_{i}, d_{G_{i}^{j+1}}\right)$. Note also that by construction the new edge $\{x, y\}$ is not incident to any vertex in $M_{j}$. Therefore the maximum degree of $\left(V_{i}, F^{j+1}\right)$ remains $d+1$. This completes the inductive construction.

The degree of every vertex of $G_{i}^{\ell+1}$ is either $d$ or $d+1$. Add to every vertex of degree $d$ a self loop so as to obtain a $d+1$ regular graph $H_{i}=\left(V_{i}, F_{i}\right)$ without changing the induced shortest path metric. Note that (272) holds true because $D_{\ell} \neq V_{i}$.

It follows from Lemma 2.7 that for every kernel $K: X \times X \rightarrow[0, \infty)$,

$$
\gamma\left(H_{i}, K\right) \leqslant \frac{d+1}{d} \gamma\left(G_{i}, K\right) \text { and } \gamma_{+}\left(H_{i}, K\right) \leqslant \frac{d+1}{d} \gamma_{+}\left(G_{i}, K\right) .
$$

In particular, since $\left\{G_{i}\right\}_{i=1}^{\infty}$ is an expander sequence also $\left\{H_{i}\right\}_{i=1}^{\infty}$ is an expander sequence.

Assume for the sake of obtaining a contradiction that $\left\{\left(V_{i}, d_{H_{i}}\right\}_{i=1}^{\infty}\right.$ admits a coarse embedding into $\left\{\left(V_{i}, \omega \circ d_{G_{i}}\right):(i, \omega) \in \mathbb{N} \times \mathscr{C}\right\}$. Then there exist $\left\{\omega_{i}\right\}_{i=1}^{\infty} \subseteq \mathscr{C}$ and nondecreasing moduli $\alpha, \beta:[0, \infty) \rightarrow[0, \infty)$ with

$$
\lim _{t \rightarrow \infty} \alpha(t)=\infty
$$

and for every $i \in \mathbb{N}$ there exists $j(i) \in \mathbb{N}$ and $f_{i}: V_{i} \rightarrow V_{j(i)}$ satisfying

$$
\forall u, v \in V\left(H_{i}\right), \quad \alpha\left(d_{H_{i}}(u, v)\right) \leqslant \omega_{i}\left(d_{G_{j(i)}}\left(f_{i}(u), f_{i}(v)\right)\right) \leqslant \beta\left(d_{H_{i}}(u, v)\right) .
$$

Note that only the values of $\beta$ on $\mathbb{N} \cup\{0\}$ matter here, and that since $\beta(\cdot)$ serves only as an upper bound in (275) we may assume without loss of generality that the sequence $\{\beta(n)\}_{n=0}^{\infty}$ is strictly increasing.

Define

$$
h_{i} \stackrel{\text { def }}{=}\left\lfloor\frac{1}{3} \min \left\{\beta^{-1}\left(\left\lfloor\omega_{i}\left(c \log n_{j(i)}\right)\right\rfloor\right), c \log n_{i}\right\}\right\rfloor .
$$


We claim that

$$
\lim _{i \rightarrow \infty} h_{i}=\infty .
$$

Indeed, since $\left\{G_{j}\right\}_{j=1}^{\infty}$ is an expander sequence,

$$
\lambda \stackrel{\text { def }}{=} \sup _{j \in \mathbb{N}} \lambda\left(G_{j}\right)<1 .
$$

We therefore have the following bound on the diameter of $G_{i}$ (see [13]):

$$
\operatorname{diam}\left(G_{j}\right) \leqslant \frac{2 \log n_{j}}{\log (1 / \lambda)} .
$$

Observe that since $G_{j}$ has girth at least $4 c \log n_{j}$, it follows from (278) that $c \log (1 / \lambda) \leqslant 1$. It now follows from (272), (275) and (278) that

$$
\alpha\left(\frac{c}{2} \log n_{i}\right) \leqslant \omega_{i}\left(\frac{2 \log n_{j(i)}}{\log (1 / \lambda)}\right) \leqslant \frac{4}{c \log (1 / \lambda)} \omega_{i}\left(c \log n_{j(i)}\right),
$$

where in the rightmost inequality of (279) we used the fact that $\omega_{i}$ is increasing and subadditive. Due to (274) and (276)), we indeed have (277) as a consequence of (279).

Our construction ensures that $H_{i}$ contains a cycle $C \stackrel{\text { def }}{=}\left\{x_{1}, \ldots, x_{3 h_{i}}\right\}$ of length $3 h_{i}$ which is embedded isometrically into $\left(H_{i}, d_{H_{i}}\right)$. Then

$$
f_{i}(C) \stackrel{\left(\frac{\sqrt{275})}{\subseteq}\right.}{\subseteq} B_{G_{j(i)}}\left(f_{i}\left(x_{1}\right), \omega_{i}^{-1}\left(\beta\left(3 h_{i}\right)\right)\right) \stackrel{\left(\frac{\sqrt{276})}{\subseteq}\right.}{\subseteq} B_{G_{j(i)}}\left(f_{i}\left(x_{1}\right), c \log n_{j(i)}\right) .
$$

Since $c \log n_{j(i)}$ is smaller than half the girth of $G_{j(i)}$, the ball $B_{G_{j(i)}}\left(f_{i}\left(x_{1}\right), c \log n_{j(i)}\right)$ is isometric to a tree. We will now proceed to show that combined with the inclusion (280) this leads to a contraction, using a coarse version of an argument of Rabinovich and Raz [62].

Let $\bar{C}$ denote the one dimensional simplicial complex induced by $C$, i.e., in $\bar{C}$, which is isometric to the circle $\frac{3 h_{i}}{2 \pi} S^{1}$, all the edges of $C$ are present as intervals of length 1. Similarly, denote by $\bar{T}$ the one dimensional simplicial complex induced by $B_{G_{j(i)}}\left(f_{i}\left(x_{1}\right), c \log n_{j(i)}\right)$ (thus $\bar{T}$ is isometric to a metric tree). Let $\bar{f}_{i}: \bar{C} \rightarrow \bar{T}$ be the linear interpolation of $f_{i}$, i.e., the extension of $f_{i}$ to $\bar{C}$ such that for every $u, v \in C$ with $\{u, v\} \in F_{i}$ the segment $[u, v]$ is mapped onto the unique geodesic $\left[f_{i}(u), f_{i}(v)\right] \subseteq \bar{T}$ with constant speed (see e.g. the discussion preceding Theorem 2 of [53]). It follows from (275) that

$$
d_{G_{j(i)}}\left(f_{i}(u), f_{i}(v)\right) \leqslant \omega_{i}^{-1}(\beta(1))
$$

whenever $\{u, v\}$ is an edge of $H_{i}$. Hence $f_{i}$ is $\omega_{i}^{-1}(\beta(1))$-Lipschitz. Therefore $\bar{f}_{i}$ is also $\omega_{i}^{-1}(\beta(1))$-Lipschitz.

Consider the three paths

$$
\overline{f_{i}}\left(\left[x_{1}, x_{h_{i}+1}\right]\right), \overline{f_{i}}\left(\left[x_{h_{i}+1}, x_{2 h_{i}+1}\right]\right), \overline{f_{i}}\left(\left[x_{2 h_{i}+1}, x_{1}\right]\right) \subseteq \bar{T} .
$$

Arguing as in [62], since $\bar{T}$ is a metric tree, there must exist a common point

$$
p \in \overline{f_{i}}\left(\left[x_{1}, x_{h_{i}+1}\right]\right) \bigcap \overline{f_{i}}\left(\left[x_{h_{i}+1}, x_{2 h_{i}+1}\right]\right) \bigcap \overline{f_{i}}\left(\left[x_{2 h_{i}+1}, x_{1}\right]\right) .
$$

We can therefore find

$$
(\bar{a}, \bar{b}, \bar{c}) \in\left[x_{1}, x_{h_{i}+1}\right] \times\left[x_{h_{i}+1}, x_{2 h_{i}+1}\right] \times\left[x_{2 h_{i}+1}, x_{1}\right]
$$


such that

$$
f_{i}(\bar{a})=f_{i}(\bar{b})=f_{i}(\bar{c})=p .
$$

By considering the closest points to $\bar{a}, \bar{b}, \bar{c}$ in $C$, there exist $a, b, c \in C$ such that

$$
\max \left\{d_{\bar{C}}(a, \bar{a}), d_{\bar{C}}(b, \bar{b}), d_{\bar{C}}(c, \bar{c})\right\} \leqslant \frac{1}{2},
$$

and

$$
\max \left\{d_{H_{i}}(a, b), d_{H_{i}}(a, c), d_{H_{i}}(b, c)\right\} \geqslant h_{i} .
$$

Without loss of generality we may assume that $d_{H_{i}}(a, b)=d_{\bar{C}}(a, b) \geqslant h_{i}$.

Since $\bar{f}_{i}$ is $\omega_{i}^{-1}(\beta(1))$-Lipschitz and $f(\bar{a})=f(\bar{b})$,

$$
\begin{array}{r}
\alpha\left(h_{i}\right) \stackrel{\sqrt{275})}{\leqslant} \omega_{i}\left(d_{G_{j(i)}}\left(f_{i}(a), f_{i}(b)\right)\right) \leqslant \omega_{i}\left(d_{G_{j(i)}}(f(a), f(\bar{a}))+d_{G_{j(i)}}(f(b), f(\bar{b}))\right) \\
\leqslant \omega_{i}\left(2 \omega_{i}^{-1}(\beta(1)) \frac{1}{2}\right)=\beta(1) .
\end{array}
$$

The desired contradiction now follows by contrasting (274) and (277) with (281).

9.2. A metric space failing calculus for nonlinear spectral gaps. Let $\left(X, d_{X}\right)$ be a metric space and $p \in(0, \infty)$. Observe that if $A=\left(a_{i j}\right)$ is an $n \times n$ symmetric stochastic matrix then, provided $X$ contains at least two points, the fact that $\gamma_{+}\left(A, d_{X}^{p}\right)<\infty$ implies that $A$ is ergodic, and therefore

$$
\lim _{t \rightarrow \infty} \gamma_{+}\left(A^{t}, d_{X}^{p}\right)=\lim _{t \rightarrow \infty} \gamma_{+}\left(\mathscr{A}_{t}(A), d_{X}^{p}\right)=1
$$

Thus, we always have asymptotic decay of the Poincaré constants of $A^{t}$ and $\mathscr{A}_{t}(A)$ as $t \rightarrow \infty$, but for the iterative construction presented in this paper we need a quantitative variant of (282) . At the very least, we need $\left(X, d_{X}^{p}\right)$ to admit the following type of uniform decay of the Poincaré constant.

Definition 9.2 (Spaces admitting uniform decay of Poincaré constants). Let $X$ be a set and $K: X \times X \rightarrow[0, \infty)$ a kernel. Say that $(X, K)$ has the uniform decay property if for every $M \in(1, \infty)$ there exists $t \in \mathbb{N}$ and $\Gamma \in[1, \infty)$ such that for every $n \in \mathbb{N}$ and every $n \times n$ symmetric stochastic matrix $A$,

$$
\gamma_{+}(A, K) \geqslant \Gamma \Longrightarrow \gamma_{+}\left(\mathscr{A}_{t}(A), K\right) \leqslant \frac{\gamma_{+}(A, K)}{M} .
$$

We now show that there exists a metric space $\left(X, d_{X}\right)$ such that $\left(X, d_{X}^{2}\right)$ does not have the uniform decay property.

Proposition 9.3. There exist a metric space $(X, \rho)$ and a universal constant $\eta \in(0, \infty)$ with the following property. For every $n \in \mathbb{N}$ there is an $n$-vertex regular graph $G_{n}=\left(V_{n}, E_{n}\right)$ such that $\lim _{n \rightarrow \infty} \gamma_{+}\left(G_{n}, \rho^{2}\right)=\infty$, yet for every $t \in \mathbb{N}$ there exists $n_{0} \in \mathbb{N}$ such

$$
n \geqslant n_{0} \Longrightarrow \gamma_{+}\left(\mathscr{A}_{t}\left(G_{n}\right), \rho^{2}\right) \geqslant \eta \cdot \gamma_{+}\left(G_{n}, \rho^{2}\right) \text {. }
$$

Proof. Define

$$
X \stackrel{\text { def }}{=} \ell_{\infty} \cap \mathbb{Z}^{\aleph_{0}}
$$


i.e., $X$ is the set of all integer-valued bounded sequences. Consider the following metric $\rho: X \times X \rightarrow[0, \infty)$.

$$
\rho(x, y) \stackrel{\text { def }}{=} \log \left(1+\|x-y\|_{\infty}\right) .
$$

Note that $\rho$ is indeed a metric since the mapping $T:[0, \infty) \rightarrow[0, \infty)$ given by

$$
T(s) \stackrel{\text { def }}{=} \log (1+s)
$$

is concave, increasing and $T(0)=0$.

Let $G_{n}=\left(V_{n}, E_{n}\right)$ be an arbitrary sequence of constant degree expanders, i.e., $G_{n}$ is an $n$-vertex graph of degree $d$ (say $d=4$ ) satisfying

$$
C \stackrel{\text { def }}{=} \sup _{n \in \mathbb{N}} \gamma_{+}\left(G_{n},\|\cdot\|_{2}^{2}\right)<\infty
$$

We claim that

$$
\gamma_{+}\left(G_{n}, \rho^{2}\right) \lesssim(\log (1+\log n))^{2} .
$$

The goal is to prove that every $f, g: G_{n} \rightarrow X$ satisfy

$$
\frac{1}{n^{2}} \sum_{(u, v) \in V_{n} \times V_{n}} \rho(f(u), g(v))^{2} \lesssim \frac{(\log (1+\log n))^{2}}{n d} \sum_{(u, v) \in E_{n}} \rho(f(u), g(v))^{2} .
$$

To this end write

$$
S_{n} \stackrel{\text { def }}{=} f\left(V_{n}\right) \cup g\left(V_{n}\right) \subseteq \mathbb{Z}^{\aleph_{0}} .
$$

By Bourgain's embedding theorem [10], applied to the metric space $\left(S_{n}, \ell_{\infty}\right)$, there exists $\beta: S_{n} \rightarrow \ell_{2}$ satisfying

$$
\forall u, v \in V_{n}, \quad\|f(u)-g(v)\|_{\infty} \leqslant\|\beta(f(u))-\beta(g(v))\|_{2} \leqslant c(1+\log n)\|f(u)-g(v)\|_{\infty},
$$

where $c \in(1, \infty)$ is a universal constant. For every $u, v \in V_{n}$ we have

$$
\begin{aligned}
& \rho(f(u), g(v)) \stackrel{(283) \wedge(285)}{\leqslant} \log \left(1+\|\beta(f(u))-\beta(g(v))\|_{2}\right) \\
& \stackrel{(285)}{\leqslant} \log \left(1+c(1+\log n)\|f(u)-g(v)\|_{\infty}\right) \stackrel{(283)}{\lesssim} \log (1+\log n) \cdot \rho(f(u), g(v)),
\end{aligned}
$$

where in the last step of (286) we used the fact that if $f(u) \neq g(v)$ then $\|f(u)-g(v)\|_{\infty} \geqslant 1$.

As shown in [42, Remark 5.4], there exists a universal constant $\kappa>1$ and a mapping $\phi: \ell_{2} \rightarrow \ell_{2}$ such that

$$
\forall x, y \in \ell_{2}, \quad T\left(\|x-y\|_{2}\right) \leqslant\|\phi(x)-\phi(y)\|_{2} \leqslant \kappa T\left(\|x-y\|_{2}\right) .
$$

A combination of (285), (286) and (287) implies that the mapping $\psi=\phi \circ \beta: S_{n} \rightarrow \ell_{2}$ satisfies

$$
\forall u, v \in V_{n} \quad \rho(f(u), g(v)) \leqslant\|\psi(f(u))-\psi(g(v))\|_{2} \lesssim \log (1+\log n) \cdot \rho(f(u), g(v)),
$$

Since $\gamma_{+}\left(G_{n},\|\cdot\|_{2}^{2}\right) \leqslant C$, we conclude that

$$
\begin{gathered}
\frac{1}{n^{2}} \sum_{(u, v) \in V_{n} \times V_{n}} \rho(f(u), g(v))^{2} \leqslant \frac{1}{n^{2}} \sum_{(u, v) \in V_{n} \times V_{n}}\|\psi(f(u))-\psi(g(v))\|_{2}^{2} \\
\leqslant \frac{C}{n d} \sum_{(u, v) \in E_{n}}\|\psi(f(u))-\psi(g(v))\|_{2}^{2} \lesssim \frac{(\log (1+\log n))^{2}}{n d} \sum_{(u, v) \in E_{n}} \rho(f(u), g(v))^{2} .
\end{gathered}
$$


This completes the proof of (284).

We will now bound $\gamma_{+}\left(\mathscr{A}_{t}\left(G_{n}\right), \rho^{2}\right)$ from below. For this purpose it is sufficient to examine a specific embedding of the graph $\mathscr{A}_{t}\left(G_{n}\right)$ into $X$. Let $\varphi: V_{n} \rightarrow \mathbb{Z}^{\aleph_{0}}$ be an isometric embedding of the shortest path metric on $\mathscr{A}_{t}\left(G_{n}\right)$ into $\left(\mathbb{Z}^{\aleph_{0}},\|\cdot\|_{\infty}\right)$. If $\{u, v\} \in E\left(\mathscr{A}_{t}\left(G_{n}\right)\right)$ then $\rho(\varphi(u), \varphi(v))=T\left(\|\varphi(u)-\varphi(v)\|_{\infty}\right)=T(1)=1$. On the other hand, since the degree of $\mathscr{A}_{t}(G)$ is $t d^{t}$, at least half of the pairs in $V_{n} \times V_{n}$ are at distance $\gtrsim \frac{\log n}{t \log d}$ in the shortest path metric metric on $\mathscr{A}_{t}(G)$. Hence for at least half of the pairs $(u, v) \in V_{n} \times V_{n}$ we have

$$
\rho(\varphi(u), \varphi(v)) \geqslant \log \left(1+\xi \frac{\log n}{t \log d}\right)
$$

where $\xi \in(0, \infty)$ is a universal constant. If

$$
n \geqslant e^{(t \log d)^{2}}
$$

then we deduce that

$$
\gamma_{+}\left(\mathscr{A}_{t}\left(G_{n}\right), \rho^{2}\right) \geqslant \frac{\frac{1}{n^{2}} \sum_{(u, v) \in V_{n} \times V_{n}} \rho(\varphi(u), \varphi(v))^{2}}{\frac{1}{n t d^{t}} \sum_{(u, v) \in E\left(\mathscr{A}_{t}\left(G_{n}\right)\right)} \rho(\varphi(u), \varphi(v))^{2}} \gtrsim(\log (1+\log n))^{2} \stackrel{(284)}{\gtrsim} \gamma_{+}\left(G_{n}, \rho^{2}\right),
$$

thus completing the proof of Proposition 9.3.

Remark 9.4. Using Matoušek's $L_{p}$-variant of the Poincaré inequality for expanders [39], the proof of Proposition 9.3 extends mutatis mutandis to show that $\left(X, d_{X}^{p}\right)$ fails to have the uniform decay property for any $p \in(0, \infty)$.

Remark 9.5. We do not know if there exists a normed space which does not have the uniform decay property, though we conjecture that such spaces do exist, and that this even holds for $\ell_{\infty}$. Note that despite the fact that all separable metric spaces embed into $\ell_{\infty}$, we cannot formally deduce from Proposition 9.3 that $\ell_{\infty}$ satisfies the same conclusion since the uniform decay property of the Poincaré constant is not necessarily monotone when passing to subsets of metric spaces. We suspect that $\left(\ell_{1},\|\cdot\|_{1}^{2}\right)$ does have the uniform decay property despite the fact that $\ell_{1}$ does not admit an equivalent uniformly convex norm.

Acknowledgments Michael Langberg was involved in early discussions on the analysis of the zigzag product. Keith Ball helped in simplifying this analysis. We thank Steven Heilman, Michel Ledoux, Mikhail Ostrovskii and Gideon Schechtman for helpful suggestions. We are also grateful to two anonymous referees for their careful reading of this paper and many helpful comments. An extended abstract announcing parts of this work, and titled "Towards a calculus for nonlinear spectral gaps", appeared in Proceedings of the TwentyFirst Annual ACM-SIAM Symposium on Discrete Algorithms (SODA 2010). M. M. was partially supported by ISF grants 221/07 and 93/11, BSF grants 2006009 and 2010021, and a gift from Cisco Research Center. Part of this work was completed while M.M. was a member of the Institute for Advanced Study at Princeton NJ., USA. A. N. was partially supported by NSF grant CCF-0832795, BSF grants 2006009 and 2010021, the Packard Foundation and the Simons Foundation. Part of this work was completed while A. N. was visiting Université Pierre et Marie Curie, Paris, France. 


\section{REFERENCES}

[1] N. Alon, O. Schwartz, and A. Shapira. An elementary construction of constant-degree expanders. Combin. Probab. Comput., 17(3):319-327, 2008.

[2] N. Alon and J. H. Spencer. The probabilistic method. Wiley-Interscience Series in Discrete Mathematics and Optimization. John Wiley \& Sons Inc., Hoboken, NJ, third edition, 2008. With an appendix on the life and work of Paul Erdős.

[3] U. Bader, A. Furman, T. Gelander, and N. Monod. Property (T) and rigidity for actions on Banach spaces. Acta Math., 198(1):57-105, 2007.

[4] K. Ball. Markov chains, Riesz transforms and Lipschitz maps. Geom. Funct. Anal., 2(2):137-172, 1992.

[5] K. Ball, E. A. Carlen, and E. H. Lieb. Sharp uniform convexity and smoothness inequalities for trace norms. Invent. Math., 115(3):463-482, 1994.

[6] Y. Bartal, N. Linial, M. Mendel, and A. Naor. On metric ramsey-type phenomena. Ann. of Math., 162(2):643-709, 2005.

[7] W. Beckner. Inequalities in Fourier analysis. Ann. of Math. (2), 102(1):159-182, 1975.

[8] A. Bonami. Étude des coefficients de Fourier des fonctions de $L^{p}(G)$. Ann. Inst. Fourier (Grenoble), 20(fasc. 2):335-402 (1971), 1970.

[9] A. A. Borovkov and S. A. Utev. An inequality and a characterization of the normal distribution connected with it. Teor. Veroyatnost. i Primenen., 28(2):209-218, 1983.

[10] J. Bourgain. On Lipschitz embedding of finite metric spaces in Hilbert space. Israel J. Math., 52(12):46-52, 1985.

[11] M. R. Bridson and A. Haefliger. Metric spaces of non-positive curvature, volume 319 of Grundlehren der Mathematischen Wissenschaften [Fundamental Principles of Mathematical Sciences]. Springer-Verlag, Berlin, 1999.

[12] P.-A. Cherix, M. Cowling, P. Jolissaint, P. Julg, and A. Valette. Groups with the Haagerup property, volume 197 of Progress in Mathematics. Birkhäuser Verlag, Basel, 2001. Gromov's a-T-menability.

[13] F. Chung. Diameters and eigenvalues. J. Amer. Math. Soc., 2(2):187-195, 1989.

[14] T. Figiel. On the moduli of convexity and smoothness. Studia Math., 56:121-155, 1976.

[15] T. Figiel and G. Pisier. Séries aléatoires dans les espaces uniformément convexes ou uniformément lisses. C. R. Acad. Sci. Paris Sér. A, 279:611-614, 1974.

[16] J. B. Garnett and D. E. Marshall. Harmonic measure, volume 2 of New Mathematical Monographs. Cambridge University Press, Cambridge, 2008. Reprint of the 2005 original.

[17] M. Gromov. Filling Riemannian manifolds. J. Differential Geom., 18(1):1-147, 1983.

[18] M. Gromov. Asymptotic invariants of infinite groups. In Geometric group theory, Vol. 2 (Sussex, 1991), volume 182 of London Math. Soc. Lecture Note Ser., pages 1-295. Cambridge Univ. Press, Cambridge, 1993.

[19] M. Gromov. Random walk in random groups. Geom. Funct. Anal., 13(1):73-146, 2003.

[20] E. Guentner, N. Higson, and S. Weinberger. The Novikov conjecture for linear groups. Publ. Math. Inst. Hautes Études Sci., (101):243-268, 2005.

[21] S. Hoory, N. Linial, and A. Wigderson. Expander graphs and their applications. Bull. Amer. Math. Soc. (N.S.), 43(4):439-561 (electronic), 2006.

[22] R. C. James. A nonreflexive Banach space that is uniformly nonoctahedral. Israel J. Math., 18:145-155, 1974.

[23] R. C. James. Nonreflexive spaces of type 2. Israel J. Math., 30(1-2):1-13, 1978.

[24] R. C. James and J. Lindenstrauss. The octahedral problem for Banach spaces. In Proceedings of the Seminar on Random Series, Convex Sets and Geometry of Banach Spaces (Mat. Inst., Aarhus Univ., Aarhus, 1974; dedicated to the memory of E. Asplund), pages 100-120. Various Publ. Ser., No. 24, Aarhus Univ., Aarhus, 1975. Mat. Inst.

[25] N. J. Kalton. The uniform structure of Banach spaces. Math. Ann., 354(4):1247-1288, 2012.

[26] G. Kasparov and G. Yu. The coarse geometric Novikov conjecture and uniform convexity. Adv. Math., 206(1):1-56, 2006.

[27] S. Khot and A. Naor. Nonembeddability theorems via Fourier analysis. Mathematische Annalen, $334(4): 821-852,2006$. 
[28] V. Lafforgue. Un renforcement de la propriété (T). Duke Math. J., 143(3):559-602, 2008.

[29] V. Lafforgue. Propriété (T) renforcée Banachique et transformation de Fourier rapide. J. Topol. Anal., 1(3):191-206, 2009.

[30] V. Lafforgue. Propriété (T) renforcée et conjecture de Baum-Connes. In Quanta of maths, volume 11 of Clay Math. Proc., pages 323-345. Amer. Math. Soc., Providence, RI, 2010.

[31] M. Ledoux. The concentration of measure phenomenon, volume 89 of Mathematical Surveys and Monographs. American Mathematical Society, Providence, RI, 2001.

[32] J. Lindenstrauss. On the modulus of smoothness and divergent series in Banach spaces. Michigan Math. $J ., 10: 241-252,1963$.

[33] J. Lindenstrauss and L. Tzafriri. Classical Banach spaces. II, volume 97 of Ergebnisse der Mathematik und ihrer Grenzgebiete [Results in Mathematics and Related Areas]. Springer-Verlag, Berlin, 1979. Function spaces.

[34] N. Linial, E. London, and Y. Rabinovich. The geometry of graphs and some of its algorithmic applications. Combinatorica, 15(2):215-245, 1995.

[35] A. Lubotzky. Expander graphs in pure and applied mathematics. Bull. Amer. Math. Soc. (N.S.), 49:113162 (electronic), 2012.

[36] A. Lubotzky, R. Phillips, and P. Sarnak. Ramanujan graphs. Combinatorica, 8(3):261-277, 1988.

[37] F. J. MacWilliams and N. J. A. Sloane. The theory of error-correcting codes. I. North-Holland Publishing Co., Amsterdam, 1977. North-Holland Mathematical Library, Vol. 16.

[38] G. A. Margulis. Explicit group-theoretic constructions of combinatorial schemes and their applications in the construction of expanders and concentrators. Problemy Peredachi Informatsii, 24(1):51-60, 1988.

[39] J. Matoušek. On embedding expanders into $\ell_{p}$ spaces. Israel J. Math., 102:189-197, 1997.

[40] B. Maurey. Type, cotype and K-convexity. In Handbook of the geometry of Banach spaces, Vol. 2, pages 1299-1332. North-Holland, Amsterdam, 2003.

[41] B. Maurey and G. Pisier. Séries de variables aléatoires vectorielles indépendantes et propriétés géométriques des espaces de Banach. Studia Math., 58(1):45-90, 1976.

[42] M. Mendel and A. Naor. Euclidean quotients of finite metric spaces. Adv. Math., 189(2):451-494, 2004.

[43] M. Mendel and A. Naor. Metric cotype. Ann. of Math. (2), 168(1):247-298, 2008. Preliminary version in SODA '06.

[44] M. Mendel and A. Naor. Expanders with respect to Hadamard spaces and random graphs. Preprint, 2012.

[45] M. Mendel and A. Naor. Spectral calculus and Lipschitz extension for barycentric metric spaces. Preprint available at http://arxiv.org/abs/1301.3963, 2013.

[46] P.-A. Meyer. Transformations de Riesz pour les lois gaussiennes. In Seminar on probability, XVIII, volume 1059 of Lecture Notes in Math., pages 179-193. Springer, Berlin, 1984.

[47] V. D. Milman and G. Schechtman. Asymptotic theory of finite-dimensional normed spaces, volume 1200 of Lecture Notes in Mathematics. Springer-Verlag, Berlin, 1986. With an appendix by M. Gromov.

[48] A. Naor. $L_{1}$ embeddings of the Heisenberg group and fast estimation of graph isoperimetry. In Proceedings of the International Congress of Mathematicians. Volume III, pages 1549-1575, New Delhi, 2010. Hindustan Book Agency.

[49] A. Naor. An introduction to the Ribe program. Jpn. J. Math., 7(2):167-233, 2012.

[50] A. Naor. On the Banach-space-valued Azuma inequality and small-set isoperimetry of Alon-Roichman graphs. Combin. Probab. Comput., 21(4):611-622, 2012.

[51] A. Naor and Y. Rabani. Spectral inequalities on curved spaces. Preprint, 2005.

[52] A. Naor and G. Schechtman. Remarks on non linear type and Pisier's inequality. J. Reine Angew. Math., 552:213-236, 2002.

[53] A. Naor and S. Sheffield. Absolutely minimal Lipschitz extension of tree-valued mappings. Math. Ann., 354(3):1049-1078, 2012.

[54] A. Naor and L. Silberman. Poincaré inequalities, embeddings, and wild groups. Compos. Math., 147(5):1546-1572, 2011.

[55] N. Ozawa. A note on non-amenability of $\mathscr{B}\left(l_{p}\right)$ for $p=1$, 2. Internat. J. Math., 15(6):557-565, 2004.

[56] G. Pisier. Martingales with values in uniformly convex spaces. Israel J. Math., 20(3-4):326-350, 1975. 
[57] G. Pisier. Some applications of the complex interpolation method to Banach lattices. J. Analyse Math., 35:264-281, 1979.

[58] G. Pisier. Holomorphic semigroups and the geometry of Banach spaces. Ann. of Math. (2), 115(2):375392, 1982.

[59] G. Pisier. A remark on hypercontractive semigroups and operator ideals. Preprint, available at http://arxiv.org/abs/0708.3423, 2007.

[60] G. Pisier. Complex interpolation between Hilbert, Banach and operator spaces. Mem. Amer. Math. Soc., 208(978):vi+78, 2010.

[61] G. Pisier and Q. H. Xu. Random series in the real interpolation spaces between the spaces $v_{p}$. In Geometrical aspects of functional analysis (1985/86), volume 1267 of Lecture Notes in Math., pages 185-209. Springer, Berlin, 1987.

[62] Y. Rabinovich and R. Raz. Lower bounds on the distortion of embedding finite metric spaces in graphs. Discrete Comput. Geom., 19(1):79-94, 1998.

[63] O. Reingold, L. Trevisan, and S. P. Vadhan. Pseudorandom walks on regular digraphs and the RL vs. L problem. In J. M. Kleinberg, editor, STOC, pages 457-466. ACM, 2006.

[64] O. Reingold, S. Vadhan, and A. Wigderson. Entropy waves, the zig-zag graph product, and new constantdegree expanders. Ann. of Math., 155(1):157-187, 2002.

[65] J. Roe. Lectures on coarse geometry, volume 31 of University Lecture Series. American Mathematical Society, Providence, RI, 2003.

[66] E. Rozenman and S. Vadhan. Derandomized squaring of graphs. In Approximation, randomization and combinatorial optimization, volume 3624 of Lecture Notes in Comput. Sci., pages 436-447. Springer, Berlin, 2005.

[67] P. Wojtaszczyk. Banach spaces for analysts, volume 25 of Cambridge Studies in Advanced Mathematics. Cambridge University Press, Cambridge, 1991.

[68] G. Yu. The coarse Baum-Connes conjecture for spaces which admit a uniform embedding into Hilbert space. Invent. Math., 139(1):201-240, 2000.

Mathematics and Computer Science Department, Open University of Israel, 1 University Road, P.O. BOX 808 RaAnana 43107, IsRaEL

E-mail address: mendelma@gmail.com

Courant Institute, New York University, 251 Mercer Street, New York NY 10012, USA

E-mail address: naor@cims.nyu.edu 